\title{
Regioselective Synthesis of 5-Metalated 2-Pyrones by Intramolecular Oxymetalation of Carbonyl-Ene-Yne Compounds Using Indium Trihalide
}

\author{
Tetsuji Yata, ${ }^{\ddagger}$ Yuji Kita, ${ }^{\ddagger}$ Yoshihiro Nishimoto, ${ }^{* \dagger}$ and Makoto Yasuda* \\ $\dagger$ Frontier Research Base for Global Young Researchers, Center for Open Innovation Research and Education \\ (COiRE), Graduate School of Engineering, Osaka University, 2-1 Yamadaoka, Suita, Osaka 565-0871, Japan \\ \$Department of Applied Chemistry, Graduate School of Engineering, Osaka University, 2-1 Yamadaoka, Suita, \\ Osaka 565-0871, Japan
}

\section{Supporting Information}




\section{Table of Contents}

1 Observation of Zwitterion Intermediate by ${ }^{1} \mathrm{H}$ NMR Spectroscopy S3 and X-ray Crystallographic Analysis

2 Computational Analysis $\quad$ S5

3 Isolation of Organoindium Compound $\quad \mathrm{S} 16$

4 References $\quad$ S17

5 X-ray crystallographic date $\quad$ S18

$\begin{array}{lll}6 & \text { NMR spectra } & \text { S24 }\end{array}$ 


\section{Observation of Zwitterion Intermediate by ${ }^{1} \mathrm{H}$ NMR Spectroscopy and X-ray}

Crystallographic Analysis

Oxyindation of methyl (Z)-3,5-diphenylpent-2-en-4-ynoate $\mathbf{1 b}(0.501 \mathrm{mmol}, 0.131 \mathrm{~g})$ with $\mathrm{InI}_{3}$ $(0.505 \mathrm{mmol}, 0.250 \mathrm{~g})$ was carried out in toluene $(1 \mathrm{~mL})$ at room temperature for $2 \mathrm{~h}$ to give a white solid, and then the toluene was evaporated and the residual solid was dissolved in $\mathrm{CDCl}_{3}$. ${ }^{1} \mathrm{H}$ NMR spectroscopy measurements showed that the solid was mixture of two compounds, which were neither the metalated pyrone $\mathbf{3 b}$ nor the starting material $\mathbf{1 b}$ (Fig. S1). Recrystallization of the mixture from $\mathrm{CHCl}_{3}$ and heptane provided a crystal and X-ray crystallographic analysis revealed that the one of the two components was the zwitterion intermediate $4 \mathbf{b}$ (CCDC 1910563). Another could be the coordination complex $\mathbf{S 1}$ because the signal of OMe was moved to lower magnetic field than that of $\mathbf{1 b}$. The tentative assignment was shown in Fig. S1 based on ${ }^{1} \mathrm{H}$ NMR spectrum of a zwitterion reported in our previous work ${ }^{1}$, and we calculated the NMR yield of $\mathbf{4 b}$ and $\mathbf{S 1}$ by addition of 1,1,1,2-tetrachloroethane as an internal standard (4b: $0.095 \mathrm{mmol}, 19 \%$, S1: $0.396 \mathrm{mmol}, 79 \%)$.
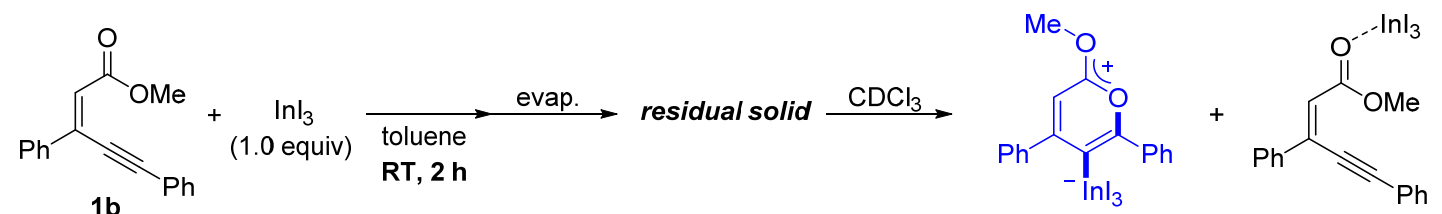
4b 19\% (NMR yield) $\quad \mathbf{S 1 7 9 \% ~ ( N M R ~ y i e l d ) ~}$

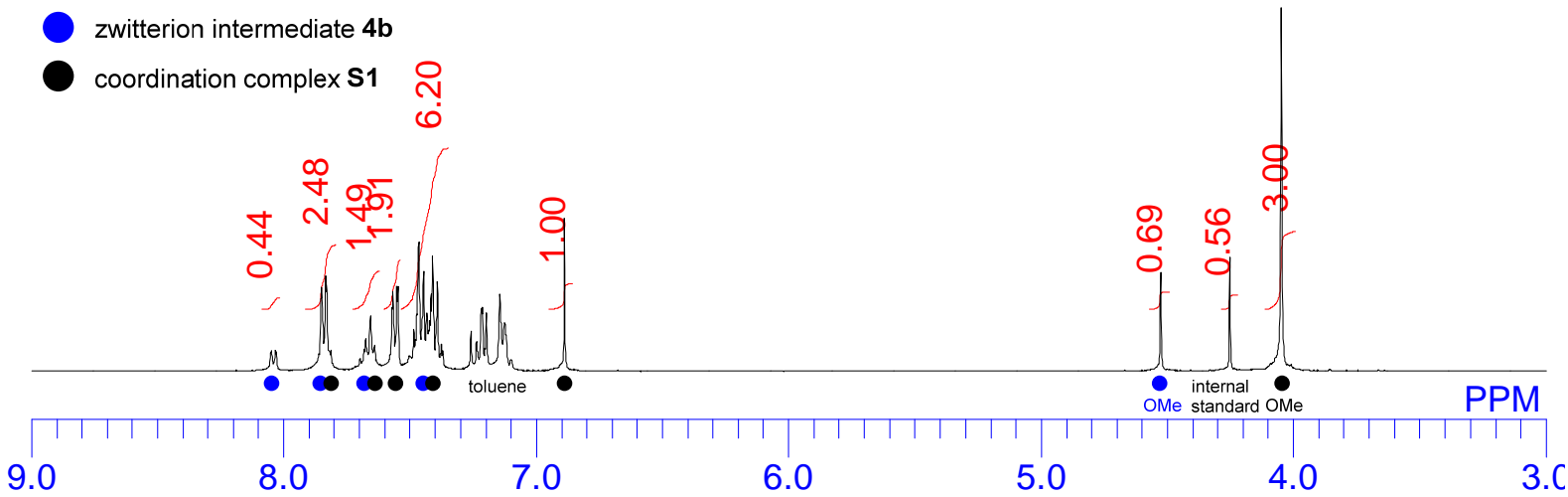

Fig. S1 ${ }^{1} \mathrm{H}$ NMR ( $400 \mathrm{MHz}, \mathrm{CDCl}_{3}$ ) spectrum of the solid obtained from oxyindation of $\mathbf{1 b}$ with $\mathrm{InI}_{3}$ at room temperature.

We tried to determine which of the signals was assigned to the zwitterion $\mathbf{4 b}$ by measurement of ${ }^{1} \mathrm{H}$ NMR spectroscopy of the crystal $\mathbf{4 b}$, but the same mixture was observed in ${ }^{1} \mathrm{H}$ NMR spectrum. This result suggested that the retro-cyclization of the zwitterion $\mathbf{4 b}$ might proceed to afford $\mathbf{S 1}$ in the solution phase (Eq. 1). 

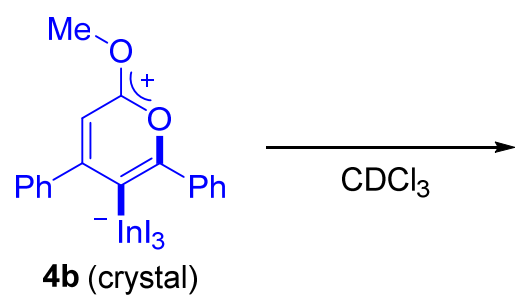<smiles></smiles>

4b

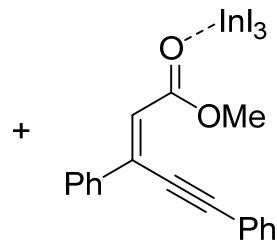

S1

The mixture of $\mathbf{4 b}(0.095 \mathrm{mmol})$ and $\mathbf{S 1}(0.396 \mathrm{mmol})$ was dissolved in toluene $(1 \mathrm{~mL})$ and the solution was heated at $80{ }^{\circ} \mathrm{C}$ in $24 \mathrm{~h}$. The reaction mixture was washed by $\mathrm{CHCl}_{3}$ to afford the metalated 2-pyrone 3b (0.244 g, 80\%) (CCDC 1910738) (Eq. 2).

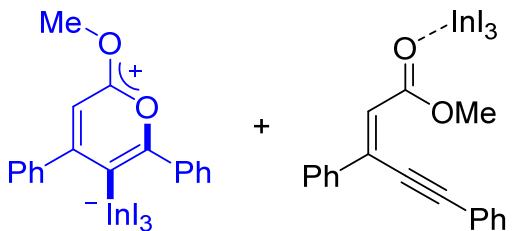

4b 19\% (NMR yield) $\quad$ S1 79\% (NMR yield) toluene

$80^{\circ} \mathrm{C}, 24 \mathrm{~h}$<smiles>O=c1cc(-c2ccccc2)c(P)c(-c2ccccc2)o1</smiles> 


\section{Computational Analysis}

We applied the HF/DFT hybrid method originally proposed by Becke, referenced as B3LYP three parameter hybrid functional. All calculations were performed with Gaussian09 rev.C.01, 6$31+\mathrm{G}(\mathrm{d}, \mathrm{p})$ for $\mathrm{H}, \mathrm{C}, \mathrm{O}$, DGDZVP for Al, Ga, In, Br were used for basis sets. All molecular geometries were fully optimized and energies were calculated including thermal free energies correction by the normal mode analysis for each structure.

Comparison of various parameters for activation of carbonyl-ene-yne 1a by metal salts ${ }^{a}$ (Table S1)

(1)

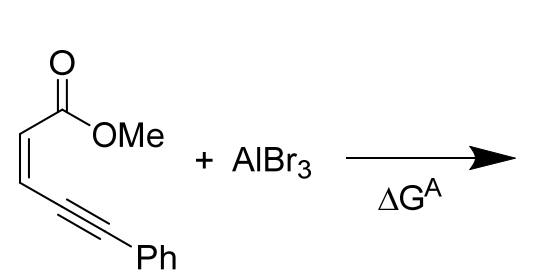

$\mathrm{Br}_{3} \mathrm{Al}$ ",

(2)<smiles>COC(=O)/C=C\C#Cc1ccccc1</smiles>

(3)<smiles>COC(=O)/C=C\C#Cc1ccccc1</smiles>

(4)<smiles>COC(=O)/C=C\C#Cc1ccccc1</smiles>

(5)<smiles>COC(=O)/C=C\C#Cc1ccccc1</smiles>

(6)<smiles>COC(=O)/C=C\C#Cc1ccccc1</smiles><smiles>COC(=O)/C=C\C#Cc1ccccc1</smiles>

$\Delta G=-37.2 \mathrm{kcal} / \mathrm{mol}$<smiles>COC(=O)/C=C\C#C[Ge](Cc1ccccc1)c1ccccc1</smiles>

$\Delta G=-12.7 \mathrm{kcal} / \mathrm{mol}$

$\mathrm{Br}_{3} \mathrm{Ga}$,<smiles>COC(=O)/C=C\C#Cc1ccccc1</smiles>

$\Delta G=-27.8 \mathrm{kcal} / \mathrm{mol}$<smiles>COC(=O)/C=C\C#CC(C)C</smiles>

$\Delta G=-12.3 \mathrm{kcal} / \mathrm{mol}$

$\mathrm{Br}_{3} \mathrm{In}$,<smiles>COC(=O)/C=C\C#Cc1ccccc1</smiles>

$\Delta G=-30.5 \mathrm{kcal} / \mathrm{mol}$

$\Delta G=-17.2 \mathrm{kcal} / \mathrm{mol}$<smiles>COC(=O)/C=C\C#C[C@@H](Br)c1ccccc1</smiles><smiles>C1CCCC1</smiles> 
Table S1. Computed Gibbs Free Energy for Complexation of Metal Salts with Alkyne Moiety or Carbonyl Oxygen ${ }^{a}$

\begin{tabular}{|c|c|c|c|c|}
\hline \\
\end{tabular}


Optimized structure of 1a, $\mathrm{AlBr}_{3}, \mathrm{GaBr}_{3}, \mathrm{InBr}_{3}, \mathrm{AlBr}_{3} \cdot 1 \mathrm{1a}\left(\right.$ alkyne), $\mathrm{GaBr}_{3} \cdot 1 \mathrm{1a}($ alkyne), $\operatorname{InBr}_{3} \cdot 1 \mathrm{1a}$ (alkyne), $\mathrm{AlBr}_{3} \cdot 1 \mathrm{1a}$ (carbonyl), $\mathrm{GaBr}_{3} \cdot 1 \mathrm{1a}$ (carbonyl), $\mathrm{InBr}_{3} \cdot 1 \mathrm{1a}$ (carbonyl) 1a
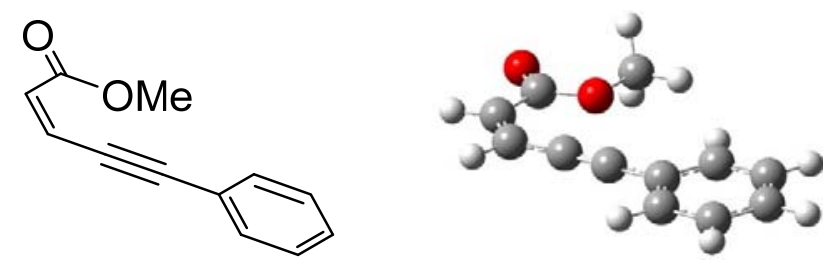

$\mathrm{AlBr}_{3}$

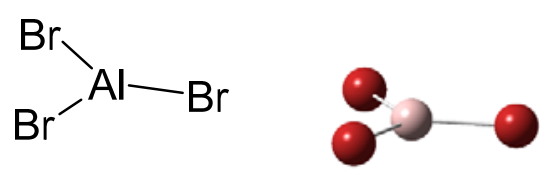

$\mathrm{GaBr}_{3}$

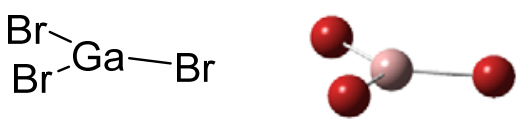

$\mathrm{InBr}_{3}$

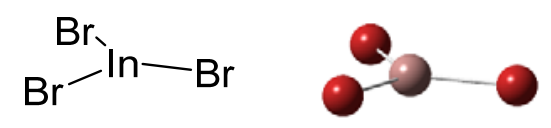

$\mathrm{AlBr}_{3} \cdot 1 \mathrm{1a}$ (alkyne)
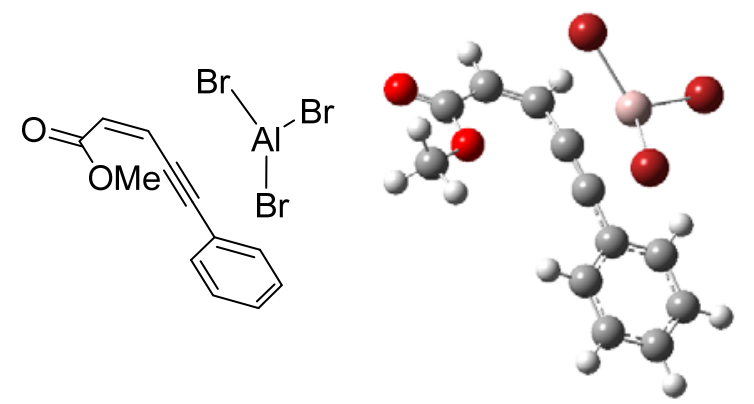

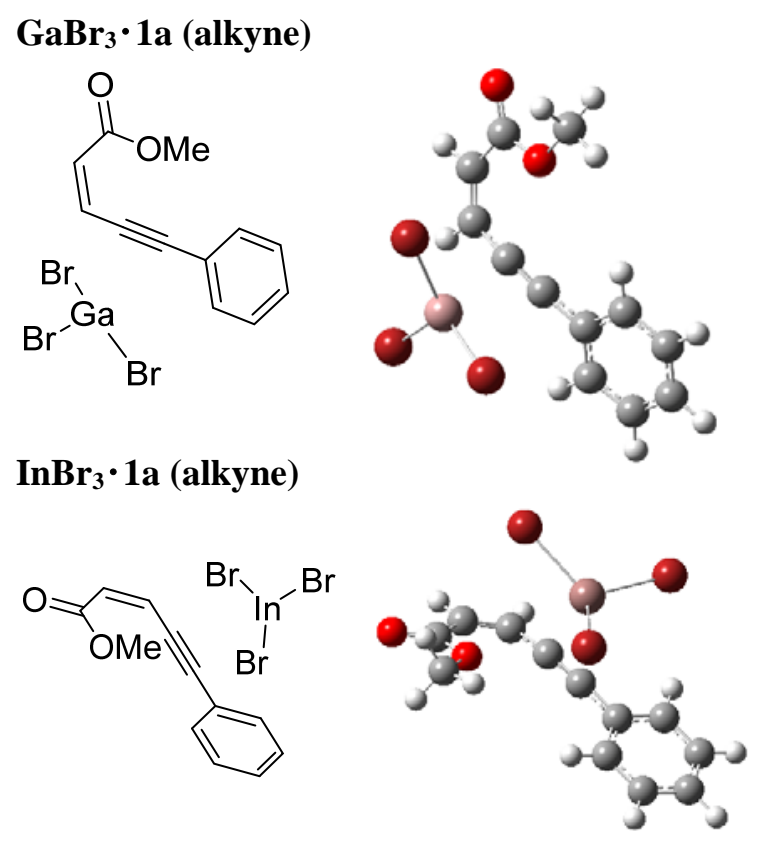

$\mathrm{AlBr}_{3} \cdot 1 \mathrm{1a}$ (carbonyl)<smiles>COC(=CC#Cc1ccccc1)O[Al](Br)Br</smiles>

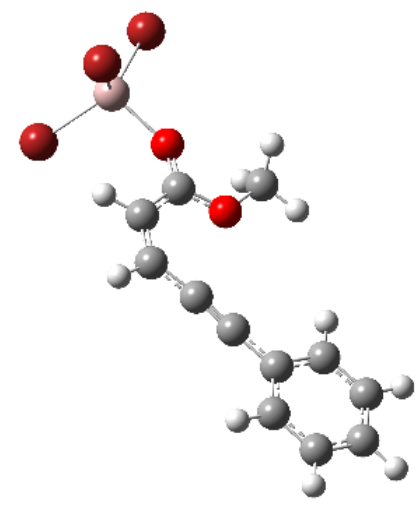

$\mathrm{GaBr}_{3} \cdot 1 \mathrm{1a}$ (carbonyl)<smiles>COC(=O)/C=C\C#Cc1ccccc1</smiles>

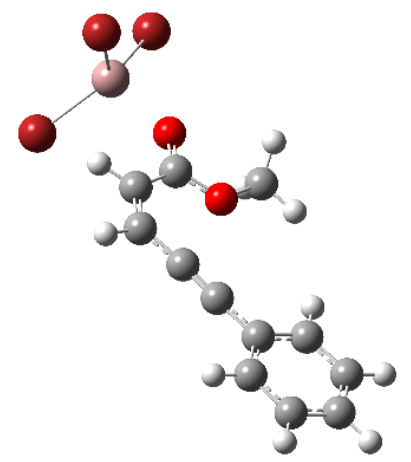


$\mathrm{InBr}_{3} \cdot 1 \mathrm{1a}$ (carbonyl)
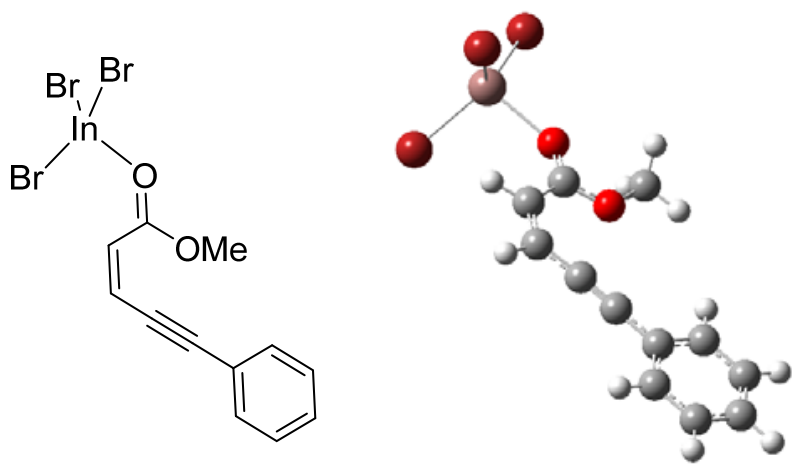

$\mathrm{AlBr}_{3}$

$\begin{array}{ll}\text { HETATM } & 1 \mathrm{Br} \\ \text { HETATM } & 2 \mathrm{Br} \\ \text { HETATM } & 3 \mathrm{Br} \\ \text { HETATM } & 4 \mathrm{Al}\end{array}$

$\odot$

$-0.000$

2. 253

0.000

$\mathrm{Br}$

$\odot$

$-1.951$

$-1.127$

$-0.000$

$\mathrm{Br}$

$\odot$

1.951

$-1.127$

$-0.000$

$\mathrm{Br}$

0

0.000

0.000

0.000

Al

\section{$\mathrm{GaBr}_{3}$}

$\begin{array}{ll}\text { HETATM } & 1 \mathrm{Ga} \\ \text { HETATM } & 2 \mathrm{Br} \\ \text { HETATM } & 3 \mathrm{Br} \\ \text { HETATM } & 4 \mathrm{Br}\end{array}$

$\odot$

$$
0.000
$$

0.000

$\odot .0 \odot \odot$

$\mathrm{Ga}$

0

$-0.000$

2. 295

0.000

$\mathrm{Br}$

$\odot$

$-1.988$

$-1.148$

$-\odot .0 \odot \odot$

$\mathrm{Br}$

$\odot$

1.988

$-1.148-0.000$

$\mathrm{Br}$

$\mathrm{InBr}_{3}$

$\begin{array}{ll}\text { HETATM } & 1 \mathrm{In} \\ \text { HETATM } & 2 \mathrm{Br} \\ \text { HETATM } & 3 \mathrm{Br} \\ \text { HETATM } & 4 \mathrm{Br}\end{array}$

0

0.000

0.000

$\odot .00 \odot$

In

$0 \quad-0.000$

2.492

$\odot .000$

$\mathrm{Br}$

$\odot$

$-2.159$

$-1.246$

$-0.000$

$\mathrm{Br}$

$\odot$

2.15

$-1.246$

$-0.000$

$\mathrm{Br}$

$1 a$

$\begin{array}{lrr}\text { HETATM } & 1 & \mathrm{C} \\ \text { HETATM } & 2 & \mathrm{C} \\ \text { HETATM } & 3 & \mathrm{C} \\ \text { HETATM } & 4 & \mathrm{C} \\ \text { HETATM } & 5 & \mathrm{C} \\ \text { HETATM } & 6 & \mathrm{C} \\ \text { HETATM } & 7 & \mathrm{H} \\ \text { HETATM } & 8 & \mathrm{H} \\ \text { HETATM } & 9 & \mathrm{H} \\ \text { HETATM } & 10 & \mathrm{H} \\ \text { HETATM } & 11 & \mathrm{H}\end{array}$

$\begin{array}{rrrr}4.345 & -0.661 & -\odot .0 \odot 1 & \mathrm{C} \\ 4.519 & 0.727 & 0.0 \odot \odot & \mathrm{C} \\ 3.402 & 1.570 & 0.0 \odot 1 & \mathrm{C} \\ 2.117 & 1.032 & 0.001 & \mathrm{C} \\ 1.930 & -0.367 & 0.0 \odot \odot & \mathrm{C} \\ 3.063 & -1.208 & -\odot .0 \odot 1 & \mathrm{C} \\ 5.210 & -1.318 & -\odot .0 \odot 1 & \mathrm{H} \\ 5.520 & 1.150 & 0.0 \odot \odot & \mathrm{H} \\ 3.534 & 2.648 & 0.0 \odot 1 & \mathrm{H} \\ 1.246 & 1.679 & 0.0 \odot 1 & \mathrm{H} \\ 2.924 & -2.285 & -\odot .0 \odot 1 & \mathrm{H}\end{array}$




$\begin{array}{lll}\text { HETATM } & 12 & \text { C } \\ \text { HETATM } & 13 & \text { C } \\ \text { HETATM } & 14 & \text { C } \\ \text { HETATM } & 15 & \text { C } \\ \text { HETATM } & 16 & \text { H } \\ \text { HETATM } & 17 & \text { H } \\ \text { HETATM } & 18 & \text { C } \\ \text { HETATM } & 19 & 0 \\ \text { HETATM } & 20 & 0 \\ \text { HETATM } & 21 & \text { C } \\ \text { HETATM } & 22 & \text { H } \\ \text { HETATM } & 23 & \text { H } \\ \text { HETATM } & 24 & \text { H }\end{array}$

$\begin{array}{rrrr}0.617 & -0.919 & 0.00 \odot & \mathrm{C} \\ -0.523 & -1.350 & 0.00 \odot & \mathrm{C} \\ -1.786 & -1.982 & 0.00 \odot & \mathrm{C} \\ -3.025 & -1.433 & 0.00 \odot & \mathrm{C} \\ -1.743 & -3.072 & 0.000 & \mathrm{H} \\ -3.883 & -2.097 & 0.00 \odot & \mathrm{H} \\ -3.412 & -0.009 & 0.00 \odot & \mathrm{C} \\ -4.578 & 0.354 & 0.001 & \mathrm{O} \\ -2.372 & 0.852 & -0.001 & \mathrm{O} \\ -2.720 & 2.247 & -0.001 & \mathrm{C} \\ -3.306 & 2.495 & -0.889 & \mathrm{H} \\ -3.301 & 2.496 & 0.891 & \mathrm{H} \\ -1.771 & 2.783 & -0.004 & \mathrm{H}\end{array}$

\section{$\mathrm{AlBr}_{3} \cdot 1 \mathrm{a}$ (alkyne)}

\begin{tabular}{|c|c|c|c|c|c|c|c|}
\hline HETATM & 1 & C & 0 & $\odot .416$ & 0.716 & -1.266 & C \\
\hline HETATM & 2 & C & $\odot$ & -0.744 & 1.024 & -0.990 & C \\
\hline HETATM & 3 & C & $\odot$ & 2.769 & 1.337 & -1.841 & C \\
\hline HETATM & 4 & C & 0 & 1.637 & 0.626 & -2.013 & C \\
\hline HETATM & 5 & $\mathrm{H}$ & 0 & 3.591 & 1.170 & -2.528 & $\mathrm{H}$ \\
\hline HETATM & 6 & $\mathrm{H}$ & 0 & 1.612 & -0.107 & -2.817 & $\mathrm{H}$ \\
\hline HETATM & 7 & C & $\odot$ & -2.079 & 1.391 & -0.684 & C \\
\hline HETATM & 8 & C & $\odot$ & -2.332 & 2.460 & 0.204 & C \\
\hline HETATM & 9 & C & 0 & -3.643 & 2.842 & $\odot .469$ & C \\
\hline HETATM & 10 & C & 0 & -4.707 & 2.165 & -0.140 & C \\
\hline HETATM & 11 & C & 0 & -4.463 & 1.104 & -1.019 & C \\
\hline HETATM & 12 & C & $\odot$ & -3.156 & 0.714 & -1.297 & C \\
\hline HETATM & 13 & $\mathrm{H}$ & $\odot$ & -1.498 & 2.967 & 0.676 & $\mathrm{H}$ \\
\hline HETATM & 14 & $\mathrm{H}$ & 0 & -3.839 & 3.662 & 1.152 & $\mathrm{H}$ \\
\hline HETATM & 15 & $\mathrm{H}$ & 0 & -5.729 & 2.464 & $\odot .074$ & $\mathrm{H}$ \\
\hline HETATM & 16 & $\mathrm{H}$ & 0 & -5.292 & 0.580 & -1.484 & $\mathrm{H}$ \\
\hline HETATM & 17 & $\mathrm{H}$ & $\odot$ & -2.952 & -0.113 & -1.969 & $\mathrm{H}$ \\
\hline HETATM & 18 & C & $\odot$ & 3.087 & 2.340 & -0.797 & C \\
\hline HETATM & 19 & 0 & 0 & 4.149 & 2.937 & -0.772 & 0 \\
\hline HETATM & 20 & 0 & 0 & 2.110 & 2.513 & $\odot .115$ & 0 \\
\hline HETATM & 21 & C & 0 & 2.411 & 3.429 & 1.187 & C \\
\hline HETATM & 22 & $\mathrm{H}$ & $\odot$ & 3.296 & 3.094 & 1.732 & $\mathrm{H}$ \\
\hline HETATM & 23 & $\mathrm{H}$ & $\odot$ & 2.587 & 4.432 & $\odot .790$ & $\mathrm{H}$ \\
\hline HETATM & 24 & $\mathrm{H}$ & 0 & 1.534 & 3.415 & 1.834 & $\mathrm{H}$ \\
\hline HETATM & 25 & $\mathrm{Al}$ & 0 & 0.174 & -1.186 & $\odot .238$ & $\mathrm{Al}$ \\
\hline HETATM & 26 & $\mathrm{Br}$ & 0 & 2.389 & -1.563 & 0.678 & $\mathrm{Br}$ \\
\hline
\end{tabular}




\begin{tabular}{|c|c|c|c|c|c|c|c|}
\hline HETATM & 27 & $\mathrm{Br}$ & 0 & -0.791 & -2.662 & -1.241 & $\mathrm{Br}$ \\
\hline HETATM & 28 & $\mathrm{Br}$ & $\Theta$ & -1.050 & -0.650 & 2.101 & $\mathrm{Br}$ \\
\hline \multicolumn{8}{|c|}{$\mathrm{GaBr}_{3} \cdot 1 \mathrm{a}$ (alkyne) } \\
\hline HETATM & 1 & $\mathrm{C}$ & 0 & $\odot .497$ & 0.883 & 1.261 & C \\
\hline HETATM & 2 & $\mathrm{C}$ & 0 & -0.649 & 1.228 & $\odot .986$ & C \\
\hline HETATM & 3 & C & $\Theta$ & 2.937 & 1.260 & 1.636 & $\mathrm{C}$ \\
\hline HETATM & 4 & $\mathrm{C}$ & $\odot$ & 1.761 & 0.642 & 1.874 & $\mathrm{C}$ \\
\hline HETATM & 5 & $\mathrm{H}$ & $\Theta$ & 3.803 & 0.955 & 2.213 & $\mathrm{H}$ \\
\hline HETATM & 6 & $\mathrm{H}$ & 0 & 1.749 & -0.147 & 2.624 & $\mathrm{H}$ \\
\hline HETATM & 7 & C & 0 & -1.957 & 1.702 & $\odot .697$ & $\mathrm{C}$ \\
\hline HETATM & 8 & C & $\Theta$ & -3.068 & 1.195 & 1.405 & $\mathrm{C}$ \\
\hline HETATM & 9 & C & 0 & -4.343 & 1.687 & 1.138 & $C$ \\
\hline HETATM & 10 & $C$ & 0 & -4.524 & 2.681 & 0.169 & $C$ \\
\hline HETATM & 11 & $C$ & 0 & -3.426 & 3.188 & -0.536 & $\mathrm{C}$ \\
\hline HETATM & 12 & $C$ & 0 & -2.147 & 2.704 & -0.278 & $\mathrm{C}$ \\
\hline HETATM & 13 & $\mathrm{H}$ & 0 & -2.918 & 0.420 & 2.149 & $\mathrm{H}$ \\
\hline HETATM & 14 & $\mathrm{H}$ & 0 & -5.196 & 1.293 & 1.681 & $\mathrm{H}$ \\
\hline HETATM & 15 & $\mathrm{H}$ & 0 & -5.521 & 3.059 & -0.037 & $\mathrm{H}$ \\
\hline HETATM & 16 & $\mathrm{H}$ & 0 & -3.571 & 3.957 & -1.288 & $\mathrm{H}$ \\
\hline HETATM & 17 & $\mathrm{H}$ & 0 & -1.289 & 3.085 & -0.821 & $\mathrm{H}$ \\
\hline HETATM & 18 & C & 0 & 3.253 & 2.324 & 0.655 & $C$ \\
\hline HETATM & 19 & 0 & 0 & 4.380 & 2.772 & $\odot .527$ & 0 \\
\hline HETATM & 20 & 0 & 0 & 2.198 & 2.741 & -0.071 & 0 \\
\hline HETATM & 21 & $C$ & 0 & 2.485 & 3.749 & -1.061 & $C$ \\
\hline HETATM & 22 & $\mathrm{H}$ & 0 & 2.877 & 4.650 & -0.582 & $\mathrm{H}$ \\
\hline HETATM & 23 & $\mathrm{H}$ & 0 & 3.217 & 3.375 & -1.780 & $\mathrm{H}$ \\
\hline HETATM & 24 & $\mathrm{H}$ & 0 & 1.534 & 3.953 & -1.551 & $\mathrm{H}$ \\
\hline HETATM & 25 & $\mathrm{Ga}$ & 0 & $\odot .059$ & -1.225 & -0.231 & $\mathrm{Ga}$ \\
\hline HETATM & 26 & $\mathrm{Br}$ & 0 & 0.017 & -2.663 & 1.609 & $\mathrm{Br}$ \\
\hline HETATM & 27 & $\mathrm{Br}$ & 0 & 2.037 & -1.066 & -1.443 & $\mathrm{Br}$ \\
\hline HETATM & 28 & $\mathrm{Br}$ & 0 & -1.885 & -1.133 & -1.503 & $\mathrm{Br}$ \\
\hline
\end{tabular}

$\mathrm{InBr}_{3} \cdot 1 \mathrm{a}$ (alkyne)

$\begin{array}{lll}\text { HETATM } & 1 & \text { C } \\ \text { HETATM } & 2 & \text { C } \\ \text { HETATM } & 3 & \text { C } \\ \text { HETATM } & 4 & \text { C } \\ \text { HETATM } & 5 & \text { H } \\ \text { HETATM } & 6 & \text { H } \\ \text { HETATM } & 7 & \text { C }\end{array}$

$\begin{array}{rrrr}0.120 & 0.983 & -1.704 & \mathrm{C} \\ -1.044 & 1.114 & -1.326 & \mathrm{C} \\ 2.479 & 1.622 & -2.220 & \mathrm{C} \\ 1.308 & 1.011 & -2.495 & \mathrm{C} \\ 3.274 & 1.576 & -2.956 & \mathrm{H} \\ 1.226 & 0.473 & -3.439 & \mathrm{H} \\ -2.387 & 1.305 & -0.905 & \mathrm{C}\end{array}$




$\begin{array}{lrrrrrrr}\text { HETATM } & 8 & \mathrm{C} & \odot & -2.696 & 2.280 & 0.068 & \mathrm{C} \\ \text { HETATM } & 9 & \mathrm{C} & \odot & -4.02 \odot & 2.49 \odot & 0.441 & \mathrm{C} \\ \text { HETATM } & 1 \odot & \mathrm{C} & \odot & -5.042 & 1.733 & -0.143 & \mathrm{C} \\ \text { HETATM } & 11 & \mathrm{C} & \odot & -4.743 & 0.762 & -1.106 & \mathrm{C} \\ \text { HETATM } & 12 & \mathrm{C} & \odot & -3.423 & 0.543 & -1.49 \odot & \mathrm{C} \\ \text { HETATM } & 13 & \mathrm{H} & \odot & -1.894 & 2.853 & 0.521 & \mathrm{H} \\ \text { HETATM } & 14 & \mathrm{H} & \odot & -4.256 & 3.238 & 1.191 & \mathrm{H} \\ \text { HETATM } & 15 & \mathrm{H} & \odot & -6.074 & 1.898 & 0.154 & \mathrm{H} \\ \text { HETATM } & 16 & \mathrm{H} & \odot & -5.538 & 0.173 & -1.553 & \mathrm{H} \\ \text { HETATM } & 17 & \mathrm{H} & \odot & -3.177 & -0.214 & -2.226 & \mathrm{H} \\ \text { HETATM } & 18 & \mathrm{In} & \odot & 0.272 & -0.881 & 0.263 & \mathrm{In} \\ \text { HETATM } & 19 & \mathrm{C} & \odot & 2.855 & 2.385 & -1.007 & \mathrm{C} \\ \text { HETATM } & 2 \odot & \mathrm{O} & \odot & 3.910 & 2.987 & -0.918 & \mathrm{O} \\ \text { HETATM } & 21 & \mathrm{O} & \odot & 1.931 & 2.339 & -0.028 & \mathrm{O} \\ \text { HETATM } & 22 & \mathrm{C} & \odot & 2.276 & 3.027 & 1.195 & \mathrm{C} \\ \text { HETATM } & 23 & \mathrm{H} & \odot & 3.200 & 2.615 & 1.607 & \mathrm{H} \\ \text { HETATM } & 24 & \mathrm{H} & \odot & 2.407 & 4.094 & 1.00 \odot & \mathrm{H} \\ \text { HETATM } & 25 & \mathrm{H} & \odot & 1.441 & 2.847 & 1.871 & \mathrm{H} \\ \text { HETATM } & 26 & \mathrm{Br} & \odot & 2.730 & -1.410 & 0.084 & \mathrm{Br} \\ \text { HETATM } & 27 & \mathrm{Br} & \odot & -1.254 & -2.649 & -0.711 & \mathrm{Br} \\ \text { HETATM } & 28 & \mathrm{Br} & \odot & -0.469 & 0.215 & 2.418 & \mathrm{Br}\end{array}$

\section{$\mathrm{AlBr}_{3} \cdot 1 \mathrm{a}$ (carbonyl)}

$\begin{array}{lrl}\text { HETATM } & 1 & \mathrm{C} \\ \text { HETATM } & 2 & \mathrm{C} \\ \text { HETATM } & 3 & \mathrm{C} \\ \text { HETATM } & 4 & \mathrm{C} \\ \text { HETATM } & 5 & \mathrm{C} \\ \text { HETATM } & 6 & \mathrm{C} \\ \text { HETATM } & 7 & \mathrm{H} \\ \text { HETATM } & 8 & \mathrm{H} \\ \text { HETATM } & 9 & \mathrm{H} \\ \text { HETATM } & 10 & \mathrm{H} \\ \text { HETATM } & 11 & \mathrm{H} \\ \text { HETATM } & 12 & \mathrm{C} \\ \text { HETATM } & 13 & \mathrm{C} \\ \text { HETATM } & 14 & \mathrm{C} \\ \text { HETATM } & 15 & \mathrm{C} \\ \text { HETATM } & 16 & \mathrm{H} \\ \text { HETATM } & 17 & \mathrm{H} \\ \text { HETATM } & 18 & \mathrm{C}\end{array}$

\begin{tabular}{|c|c|c|}
\hline-8.018 & -0.631 & -0.000 \\
\hline-8.233 & 0.752 & -0.000 \\
\hline-7.144 & 1.631 & $\odot . ๑ \odot \odot$ \\
\hline-5.843 & 1.134 & $\odot . ๑ \odot \odot$ \\
\hline-5.616 & -0.260 & 0.000 \\
\hline-6.721 & -1.139 & $-\odot .0 \odot \odot$ \\
\hline-8.863 & -1.313 & $-\odot . \odot \odot \odot$ \\
\hline-9.246 & 1.143 & -0.000 \\
\hline-7.311 & 2.704 & $\odot .000$ \\
\hline-4.994 & 1.808 & 0.000 \\
\hline-6.548 & -2.210 & -0.000 \\
\hline-4.292 & -0.773 & $\odot .00 \odot$ \\
\hline-3.143 & -1.189 & $\odot .000$ \\
\hline-1.879 & -1.797 & $\odot .0 \odot \odot$ \\
\hline-0.639 & -1.231 & 0.000 \\
\hline-1.899 & -2.887 & 0.000 \\
\hline 0.228 & -1.881 & $\odot .000$ \\
\hline-0.332 & 0.183 & $-\odot .0 \odot \odot$ \\
\hline
\end{tabular}




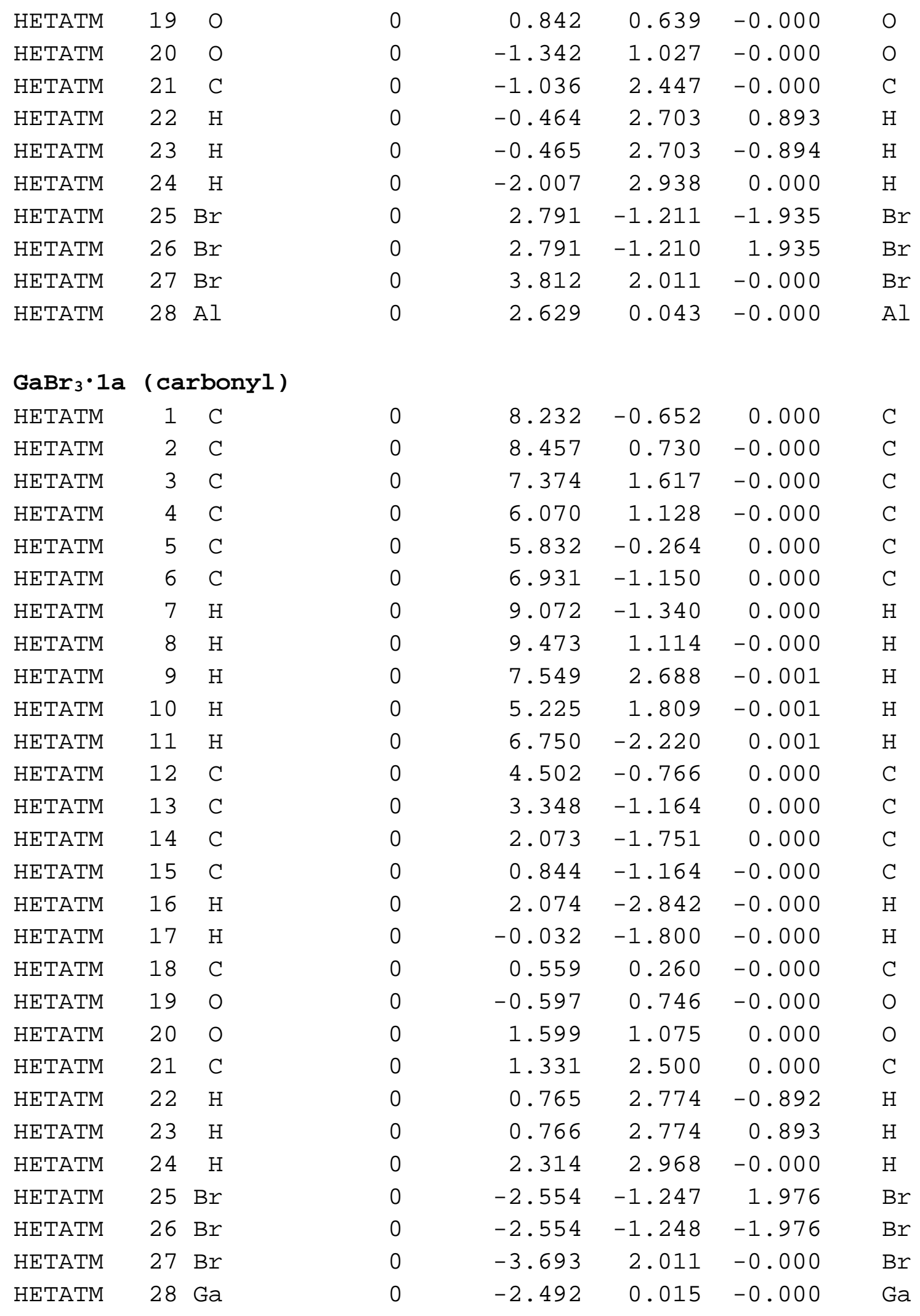


$\mathrm{InBr}_{3} \cdot 1 \mathrm{a}$ (carbonyl)

\begin{tabular}{|c|c|c|c|c|c|c|}
\hline HETATM & 1 & $C$ & $\odot$ & -8.533 & $\odot .667$ & -0.038 \\
\hline HETATM & 2 & C & $\odot$ & -8.780 & -0.710 & -0.032 \\
\hline HETATM & 3 & C & 0 & -7.712 & -1.614 & 0.000 \\
\hline HETATM & 4 & C & $\odot$ & -6.400 & -1.147 & $\odot .029$ \\
\hline HETATM & 5 & C & $\odot$ & -6.140 & 0.241 & 0.023 \\
\hline HETATM & 6 & C & $\Theta$ & -7.225 & 1.145 & -0.010 \\
\hline HETATM & 7 & $\mathrm{H}$ & $\odot$ & -9.361 & 1.369 & -0.063 \\
\hline HETATM & 8 & $\mathrm{H}$ & $\odot$ & -9.801 & -1.078 & -0.054 \\
\hline HETATM & 9 & H & $\odot$ & $-7.9 \odot 4$ & -2.683 & $\odot .004$ \\
\hline HETATM & 10 & $\mathrm{H}$ & $\odot$ & -5.566 & -1.841 & 0.054 \\
\hline HETATM & 11 & $\mathrm{H}$ & $\Theta$ & -7.026 & 2.212 & -0.015 \\
\hline HETATM & 12 & C & $\odot$ & -4.804 & 0.725 & 0.053 \\
\hline HETATM & 13 & C & 0 & -3.648 & 1.116 & 0.079 \\
\hline HETATM & 14 & C & $\odot$ & -2.375 & 1.710 & 0.107 \\
\hline HETATM & 15 & C & $\odot$ & -1.143 & 1.132 & 0.158 \\
\hline HETATM & 16 & $\mathrm{H}$ & $\odot$ & -2.383 & 2.800 & $\odot . \odot 83$ \\
\hline HETATM & 17 & $\mathrm{H}$ & $\odot$ & -0.274 & 1.779 & 0.180 \\
\hline HETATM & 18 & C & $\odot$ & -0.838 & -0.289 & 0.200 \\
\hline HETATM & 19 & 0 & $\odot$ & $\odot .326$ & -0.748 & 0.204 \\
\hline HETATM & 20 & 0 & $\odot$ & -1.870 & -1.116 & 0.239 \\
\hline HETATM & 21 & C & $\Theta$ & -1.584 & -2.536 & 0.274 \\
\hline HETATM & 22 & $\mathrm{H}$ & $\odot$ & -1.038 & -2.829 & -0.625 \\
\hline HETATM & 23 & $\mathrm{H}$ & 0 & -0.995 & -2.778 & 1.160 \\
\hline HETATM & 24 & H & $\odot$ & -2.561 & -3.016 & 0.311 \\
\hline HETATM & 25 & In & $\odot$ & 2.415 & $\odot .005$ & -0.025 \\
\hline HETATM & 26 & $\mathrm{Br}$ & $\Theta$ & 3.663 & -2.088 & 0.610 \\
\hline HETATM & 27 & $\mathrm{Br}$ & $\odot$ & 2.448 & 1.937 & 1.615 \\
\hline HETATM & 28 & $\mathrm{Br}$ & $\odot$ & 2.347 & $\odot .669$ & -2.465 \\
\hline
\end{tabular}


Table S2. Various parameters at $298.150 \mathrm{~K}$ of all optimized structures (in hartree).

\begin{tabular}{|c|c|c|c|c|c|c|c|c|c|}
\hline Compound & $\begin{array}{l}\text { Zero-point } \\
\text { correction }\end{array}$ & $\begin{array}{l}\text { Thermal } \\
\text { correction } \\
\text { to Energy }\end{array}$ & $\begin{array}{l}\text { Thermal } \\
\text { correction } \\
\text { to Enthalpy }\end{array}$ & \begin{tabular}{|l|} 
Thermal \\
correction \\
to Gibbs \\
Free \\
Energy \\
(Hartree)
\end{tabular} & $\begin{array}{lr}\text { Sum } & \text { of } \\
\text { electronic } & \text { and } \\
\text { zero-point } & \\
\text { Energies } & \\
\text { (Hartree) } & \end{array}$ & $\begin{array}{lr}\text { Sum } & \text { of } \\
\text { electronic } & \text { and } \\
\text { thermal } & \\
\text { Energies } & \\
& \end{array}$ & $\begin{array}{l}\text { Sum } \\
\text { electronic and } \\
\text { thermal } \\
\text { Enthalpies } \\
\text { (Hartree) }\end{array}$ & 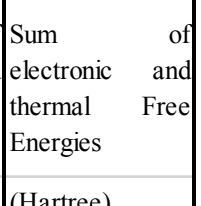 & $\begin{array}{l}\text { imaginary } \\
\text { frequency }\end{array}$ \\
\hline & (Hartree) & (Hartree) & [(Hartree) & (Hartree) & (Hartree) & (Hartree) & (Hartree) & (Hartree) & \\
\hline 1a & 0.186851 & 0.199957 & 0.200902 & 0.144145 & -613.520956 & -613.507850 & -613.506906 & -613.563662 & 0 \\
\hline $\mathrm{AlBr}_{3}$ & 0.003591 & 0.009506 & 0.010451 & -0.029145 & -7963.689214 & -7963.683298 & -7963.682354 & -7963.721950 & 0 \\
\hline $\mathrm{GaBr}_{3}$ & 0.002650 & 0.009029 & 0.009973 & -0.031083 & -9645.709776 & -9645.703398 & -9645.702454 & -9645.743509 & 0 \\
\hline $\mathrm{InBr}_{3}$ & 0.002184 & 0.008871 & 0.009816 & -0.032874 & -13463.207943 & -13463.201256 & -13463.200312 & -13463.243002 & 0 \\
\hline $\begin{array}{c}\mathrm{AlBr}_{3} \cdot \\
\text { 1a(alkyne) }\end{array}$ & 0.190885 & 0.212102 & 0.213047 & 0.131985 & -8577.217762 & -8577.196545 & -8577.195600 & -8577.276662 & 0 \\
\hline $\begin{array}{c}\mathrm{GaBr}_{3} \cdot \\
\text { 1a(alkyne) }\end{array}$ & 0.189922 & 0.211727 & 0.212672 & 0.129788 & -10259.235556 & -10259.213750 & -10259.212806 & -10259.295689 & 0 \\
\hline $\begin{array}{c}\mathrm{InBr}_{3} \cdot \\
\text { 1a(alkyne) }\end{array}$ & 0.189629 & 0.211582 & 0.212526 & 0.130453 & -14076.742098 & -14076.720145 & -14076.719201 & -14076.801274 & 0 \\
\hline $\begin{array}{c}\mathrm{AlBr}_{3} \cdot \\
\text { 1a(carbonyl) } \\
\end{array}$ & 0.192405 & 0.213029 & 0.213973 & 0.133971 & -8577.257342 & -8577.236718 & -8577.235774 & -8577.315776 & 0 \\
\hline $\begin{array}{c}\mathrm{GaBr}_{3} \cdot \\
\text { 1a(carbonyl) } \\
\end{array}$ & 0.191203 & 0.212400 & 0.213344 & 0.132290 & -10259.261547 & -10259.240349 & -10259.239405 & -10259.320460 & 0 \\
\hline $\begin{array}{c}\mathrm{InBr}_{3}{ }^{\circ} \\
\text { 1a(carbonyl) }\end{array}$ & 0.190646 & 0.212276 & 0.213220 & 0.129494 & -14076.763988 & -14076.742358 & -14076.741414 & -14076.825140 & 0 \\
\hline
\end{tabular}




\section{Isolation of Organoindium Compound}

(2-oxo-4,6-diphenyl-2H-pyran-5-yl)indium diiodide pyridine complex (3b·pyridine)<smiles>COC(=O)/C=C(/C#Cc1ccccc1)c1ccccc1</smiles>

All operations were carried out in a nitrogen-filled glove box. To a $10 \mathrm{~mL}$ vial filled with $\mathrm{InI}_{3}$ $(0.502 \mathrm{mmol}, 0.249 \mathrm{~g})$ in toluene $(1 \mathrm{~mL})$ was added methyl $(Z)$-3,5-diphenylpent-2-en-4-ynoate $(0.493 \mathrm{mmol}, 0.129 \mathrm{~g})$. The vial was sealed, and the mixture was stirred at $80{ }^{\circ} \mathrm{C}$ for $24 \mathrm{~h}$. Then, the solvent was removed by decantation to obtain a white solid and the solid was washed by $\mathrm{CHCl}_{3}$ ( $3 \mathrm{~mL} \times \mathrm{6}$ ). The residue was dried under vacuum to give the product $3 \mathbf{b}$ as a white solid $(0.297 \mathrm{~g}$, $81 \%)$. 3b was added to pyridine $(0.399 \mathrm{mmol}, 0.0316 \mathrm{~g})$ and recrystallized from $\mathrm{CHCl}_{3}$ and heptane to give a single crystal of $\mathbf{3 b}$-pyridine. The structure was determined by X-ray crystallographic analysis (CCDC 1910738). Characterization by NMR study was also carried out, and the spectra is shown below. ${ }^{1} \mathrm{H}$ NMR: $\left(400 \mathrm{MHz}, \mathrm{CDCl}_{3}\right) 8.27(\mathrm{~d}, J=4.8 \mathrm{~Hz}, 2 \mathrm{H}, 15-\mathrm{H} \times \mathrm{2})$, 7.77-7.72 (m, 3H), 7.54-7.48 (m, 2H, 8-H x 2), 7.36-7.34 (m, 6H), 7.28-7.26 (m, 2H, 16-H x 2), 6.35 (s, 1H, 3-H), ${ }^{13} \mathrm{C}$ NMR: (100 MHz, CDCl $) 167.2$ (s, C-6), 162.7 (s), 162.6 (s), 148.0 (d, C15), 141.1 (s, C-7), 139.1 (d, C-17), 136.0 (s, C-11), 131.2 (d), 130.1 (d), 129.6 (d), 129.09 (d), 129.06 (d), 127.7 (d, C-8), 124.9 (d, C-16), 118.9 (s, C-5), 111.8 (d, C-3).

${ }^{1} \mathrm{H} \mathrm{NMR}\left(400 \mathrm{MHz}, \mathrm{CDCl}_{3}\right)$

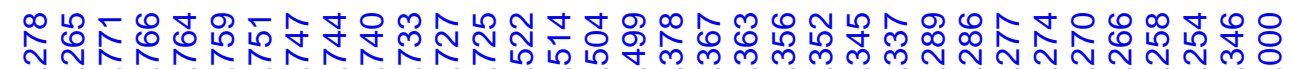

क
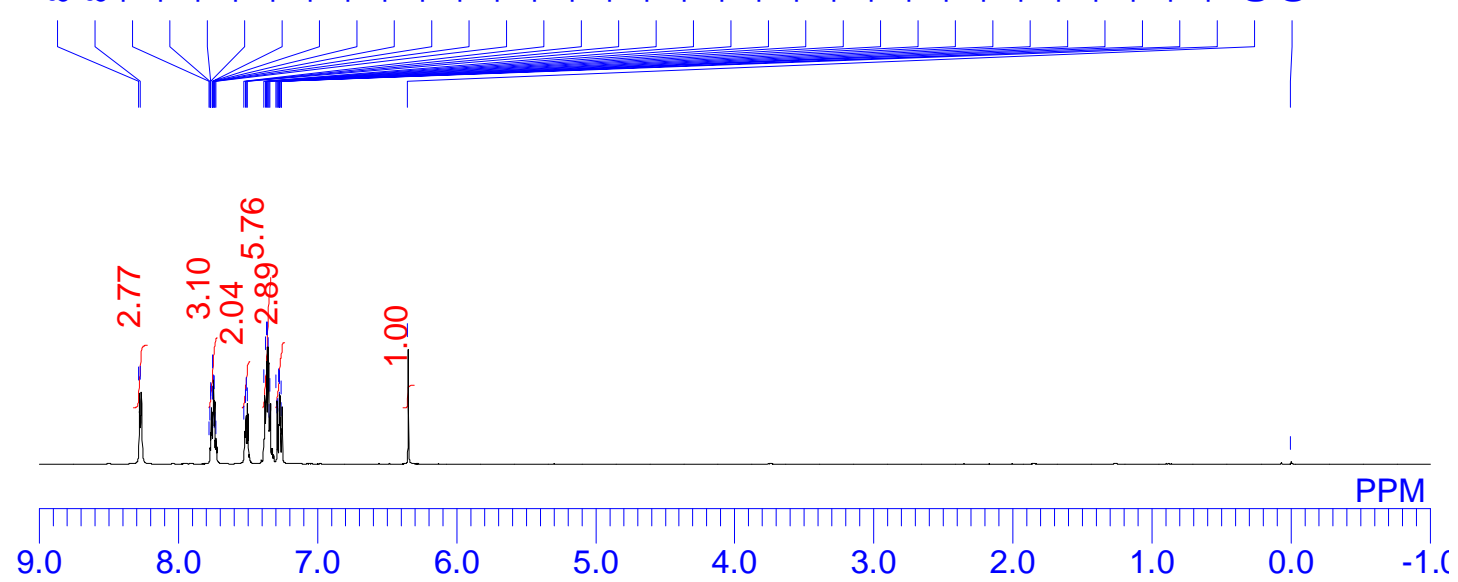
${ }^{13} \mathrm{C}\left\{{ }^{1} \mathrm{H}\right\} \mathrm{NMR}\left(100 \mathrm{MHz}, \mathrm{CDCl}_{3}\right)$

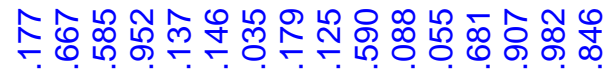

굥ㅇำ

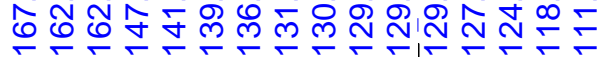

站兑
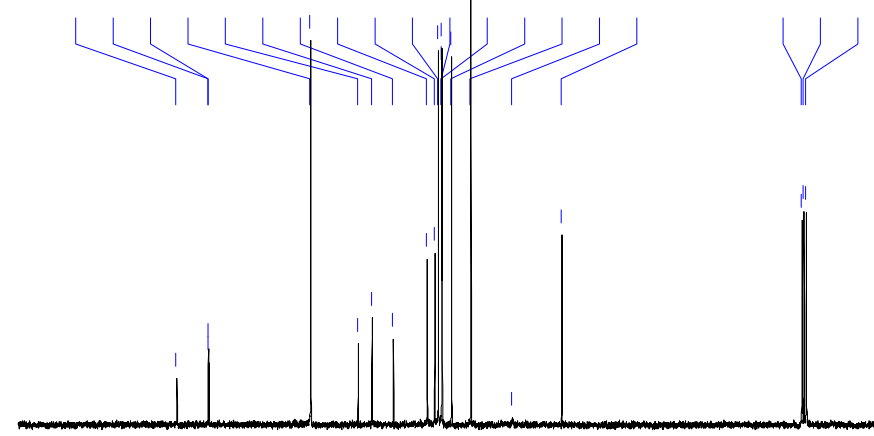

年

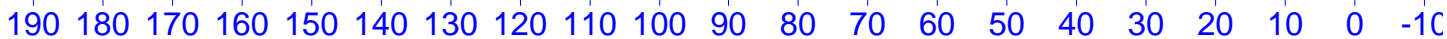

\section{References}

[1] Y. Kita, T. Yata, Y. Nishimoto, K. Chiba, M. Yasuda, Chem. Sci. 2018, 9, 6041-6052. 


\section{X-ray crystallographic date}

\section{3,4,5,6-tetraphenyl-2H-pyran-2-one (6m) (CCDC 1910558).}

$6 \mathbf{m}$ was recrystallized in dichloromethane solvent.

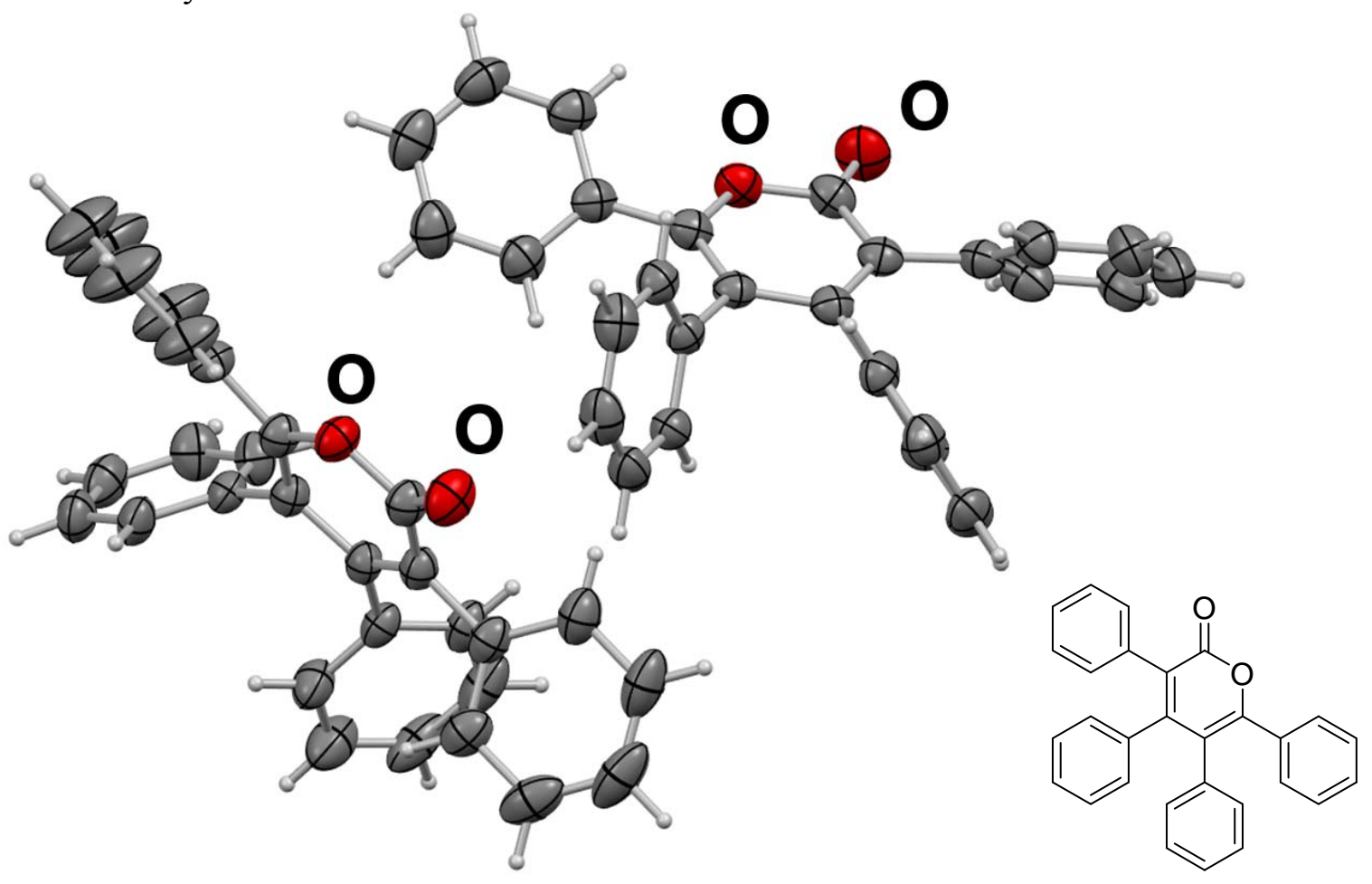

Figure S2. ORTEP drawing of $6 \mathrm{~m}$ at the $50 \%$ probability level

Table S3. Crystal date and structure solution paramaters of $6 \mathrm{~m}$

\begin{tabular}{|l|l|l|l|}
\hline Empirical Formula & $\mathrm{C}_{29} \mathrm{H}_{20} \mathrm{O}_{2}$ & Function Minimized & $\Sigma \mathrm{W}(|\mathrm{Fo}|-|\mathrm{Fc}|)^{2}$ \\
\hline Formula Weight & 400.48 & Least Squares Weights & $1 / \sigma^{2}(\mathrm{Fo})$ \\
\hline Crystal Color, Habit & None & No. Observations $(I>2.00 \sigma(I))$ & 6515 \\
\hline Crystal Dimensions & None & No. Variables & 599 \\
\hline Crystal System & triclinic & Reflection/Parameter Ratio & 10.88 \\
\hline Lattice Type & Primitive & Residuals: $R(I>2.00 \sigma(I))$ & 0.0502 \\
\hline & $a=10.2861(4) \AA$ & Residuals: $w R(I>2.00 \sigma(I))$ & 0.0453 \\
\hline & $b=12.2992(4) \AA$ & Goodness of Fit Indicator & 1.744 \\
\hline & $c=18.0217(7) \AA$ & Max Shift/Error in Final Cycle & 0.000 \\
\hline & $V=2123.76(14) \AA^{3}$ & Maximum peak in Final Diff. Map & $1.01 \mathrm{e}^{-} / \AA^{3}$ \\
\hline & $\alpha=85.011(3)^{\circ}$ & Minimum peak in Final Diff. Map & $-0.62 \mathrm{e}^{-} / \AA^{3}$ \\
\hline & $\beta=79.264(3)^{\circ}$ & & \\
\hline Space Group & $\gamma=71.536(3)^{\circ}$ & & \\
\hline$Z$ value & $P-1(\# 2)$ & & \\
\hline$D_{\text {calc }}$ & 4 & & \\
\hline$F_{000}$ & $1.252 \mathrm{~g} / \mathrm{cm}^{3}$ & & \\
\hline$\mu($ MoK $\alpha)$ & 840.00 & & \\
\hline Temperature & $0.773 \mathrm{~cm}^{-1}$ & & \\
\hline
\end{tabular}


5-(4-methylbenzoyl)-3,4,6-triphenyl-2H-pyran-2-one (7m) (CCDC 1910562).

$7 \mathbf{m}$ was recrystallized in dichloromethane solvent.

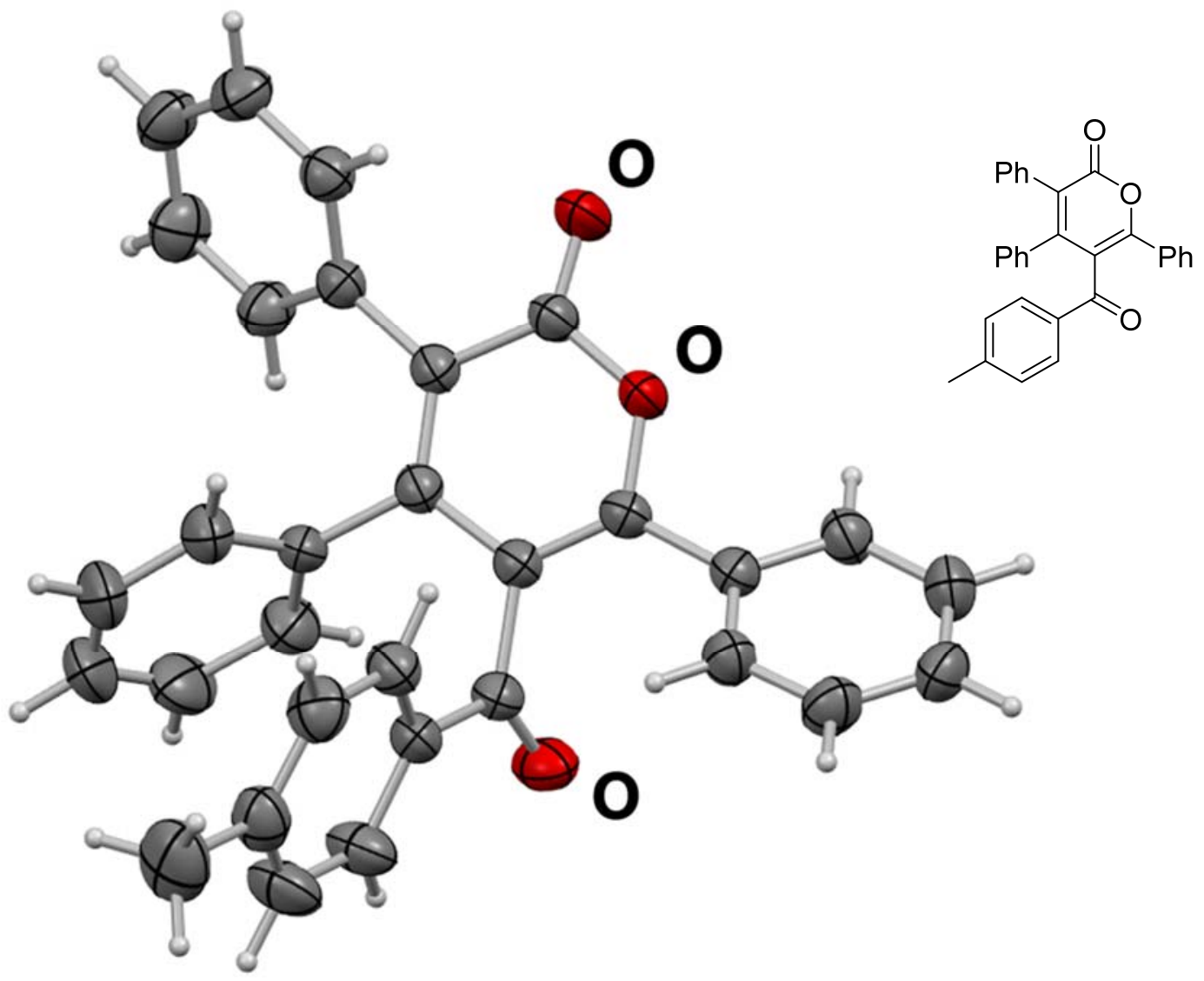

Figure S3. ORTEP drawing of $7 \mathrm{~m}$ at the $50 \%$ probability level

Table S4. Crystal date and structure solution paramaters of $7 \mathrm{~m}$

\begin{tabular}{|l|l|l|l|}
\hline Empirical Formula & $\mathrm{C}_{31} \mathrm{H}_{22} \mathrm{O}_{3}$ & Function Minimized & $\Sigma \mathrm{w}(|\mathrm{Fo}|-|\mathrm{Fc}|)^{2}$ \\
\hline Formula Weight & 442.51 & Least Squares Weights & $1 /[0.0018 \mathrm{Fo} 2+1.0000 \quad(\mathrm{Fo} 2)]$ \\
\hline Crystal Color, Habit & None & No. Observations $(I>2.00 \sigma(I))$ & 4216 \\
\hline Crystal Dimensions & None & No. Variables & 330 \\
\hline Crystal System & monoclinic & Reflection/Parameter Ratio & 12.78 \\
\hline Lattice Type & Primitive & Residuals: $R(I>2.00 \sigma(I))$ & 0.0433 \\
\hline & $a=10.4592(8) \AA$ & Residuals: $w R(I>2.00 \sigma(I))$ & 0.0581 \\
\hline & $b=8.1844(6) \AA$ & Goodness of Fit Indicator & 0.963 \\
\hline & $c=13.6348(9) \AA$ & Max Shift/Error in Final Cycle & 0.000 \\
\hline & $V=1147.48(15) \AA^{3}$ & Maximum peak in Final Diff. Map & $1.16 \mathrm{e}^{-} / \AA^{3}$ \\
\hline & $\beta=100.540(7)^{\circ}$ & Minimum peak in Final Diff. Map & $-1.06 \mathrm{e}^{-} / \AA^{3}$ \\
\hline Space Group & $P 2_{1}(\# 4)$ & & \\
\hline$Z$ value & 2 & & \\
\hline$D_{\text {calc }}$ & $1.281 \mathrm{~g} / \mathrm{cm}^{3}$ & & \\
\hline$F_{000}$ & 464.00 & & \\
\hline$\mu\left(\mathrm{MoK}_{\alpha}\right)$ & $0.814 \mathrm{~cm}^{-1}$ & & \\
\hline Temperature & $-150.0^{\circ} \mathrm{C}$ & & \\
\hline & \multicolumn{3}{l}{} \\
\hline
\end{tabular}


6-cyclopropyl-3-ethyl-5-(4-methylbenzoyl)-4-phenyl-2H-pyran-2-one (7l) (CCDC 1910561). 71 was recrystallized in dichloromethane solvent.

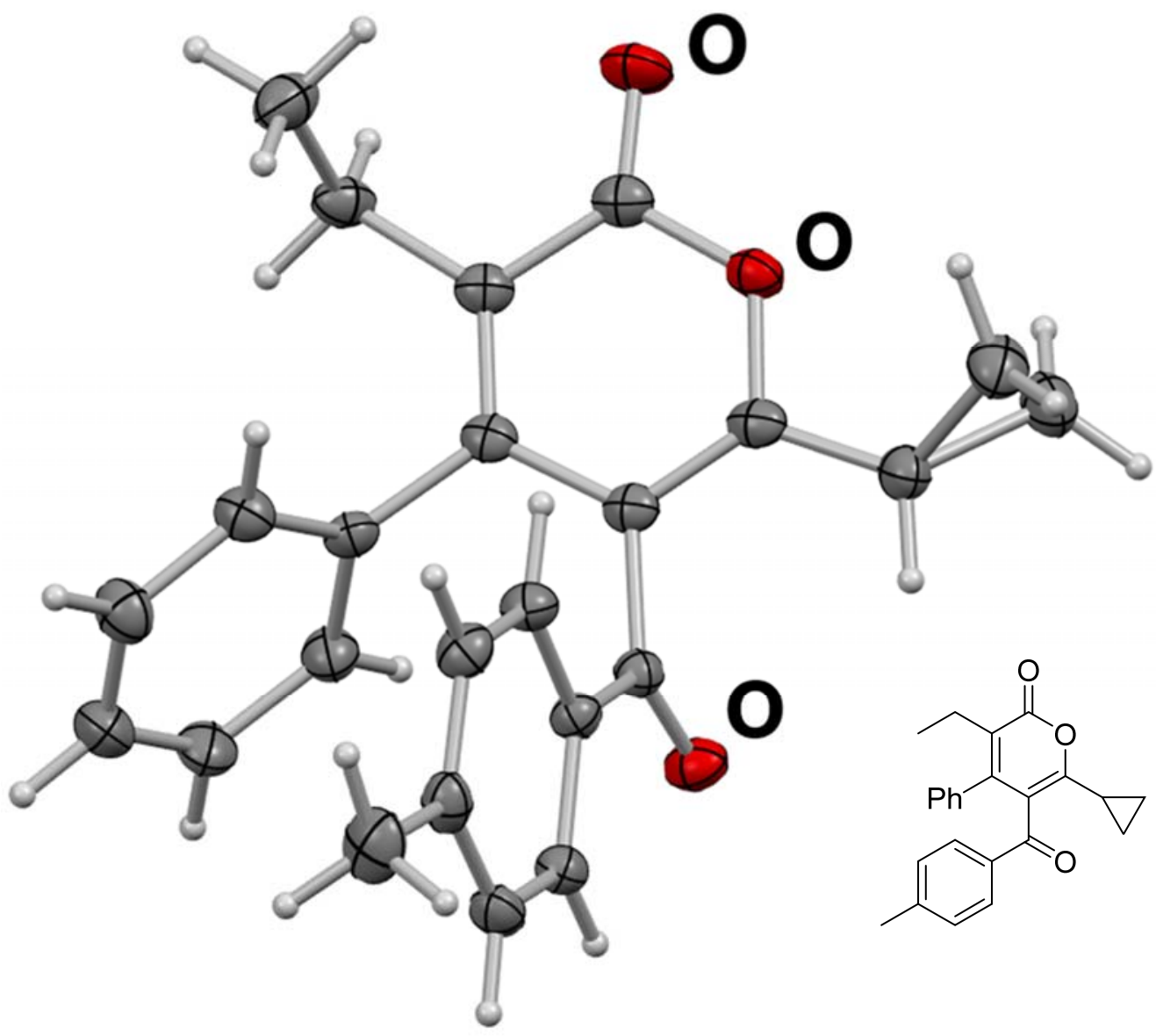

Figure S4. ORTEP drawing of 71 at the $50 \%$ probability level

Table S5. Crystal date and structure solution paramaters of $7 \mathrm{l}$

\begin{tabular}{|l|l|l|l|}
\hline Empirical Formula & $\mathrm{C}_{24} \mathrm{H}_{22} \mathrm{O}_{3}$ & Function Minimized & $\Sigma \mathrm{w}(|\mathrm{Fo}|-|\mathrm{Fc}|)^{2}$ \\
\hline Formula Weight & 358.44 & Least Squares Weights & $1 /[0.0070 \mathrm{Fo} 2+1.0000 \quad(\mathrm{Fo} 2)]$ \\
\hline Crystal Color, Habit & None & No. Observations $(I>2.00 \sigma(I))$ & 3974 \\
\hline Crystal Dimensions & None & No. Variables & 266 \\
\hline Crystal System & monoclinic & Reflection/Parameter Ratio & 14.94 \\
\hline Lattice Type & Primitive & Residuals: $R(I>2.00 \sigma(I))$ & 0.0537 \\
\hline & $a=8.4349(5) \AA$ & Residuals: $w R(I>1.00 \sigma(I))$ & 0.1004 \\
\hline & $b=10.8431(5) \AA$ & Goodness of Fit Indicator & 0.981 \\
\hline & $c=20.7594(11) \AA$ & Max Shift/Error in Final Cycle & 0.018 \\
\hline & $V=1887.95(17) \AA^{3}$ & Maximum peak in Final Diff. Map & $0.59 \mathrm{e}^{-} / \AA^{3}$ \\
\hline & $\beta=96.089(5)^{\circ}$ & Minimum peak in Final Diff. Map & $-0.76 \mathrm{e}^{-} / \AA^{3}$ \\
\hline Space Group & $P 2 / / \mathrm{c}(\# 14)$ & & \\
\hline$Z$ value & 4 & & \\
\hline$D_{\text {calc }}$ & $1.261 \mathrm{~g} / \mathrm{cm}^{3}$ & & \\
\hline$F_{000}$ & 760.00 & & \\
\hline$\mu($ MoK $\alpha)$ & $0.819 \mathrm{~cm}^{-1}$ & & \\
\hline Temperature & $-150.0^{\circ} \mathrm{C}$ & & \\
\hline & \multicolumn{3}{l}{} \\
\hline
\end{tabular}


3,4,6-triphenyl-5-(phenylethynyl)-2H-pyran-2-one (8) (CCDC 1915263).

8 was recrystallized in dichloromethane solvent.

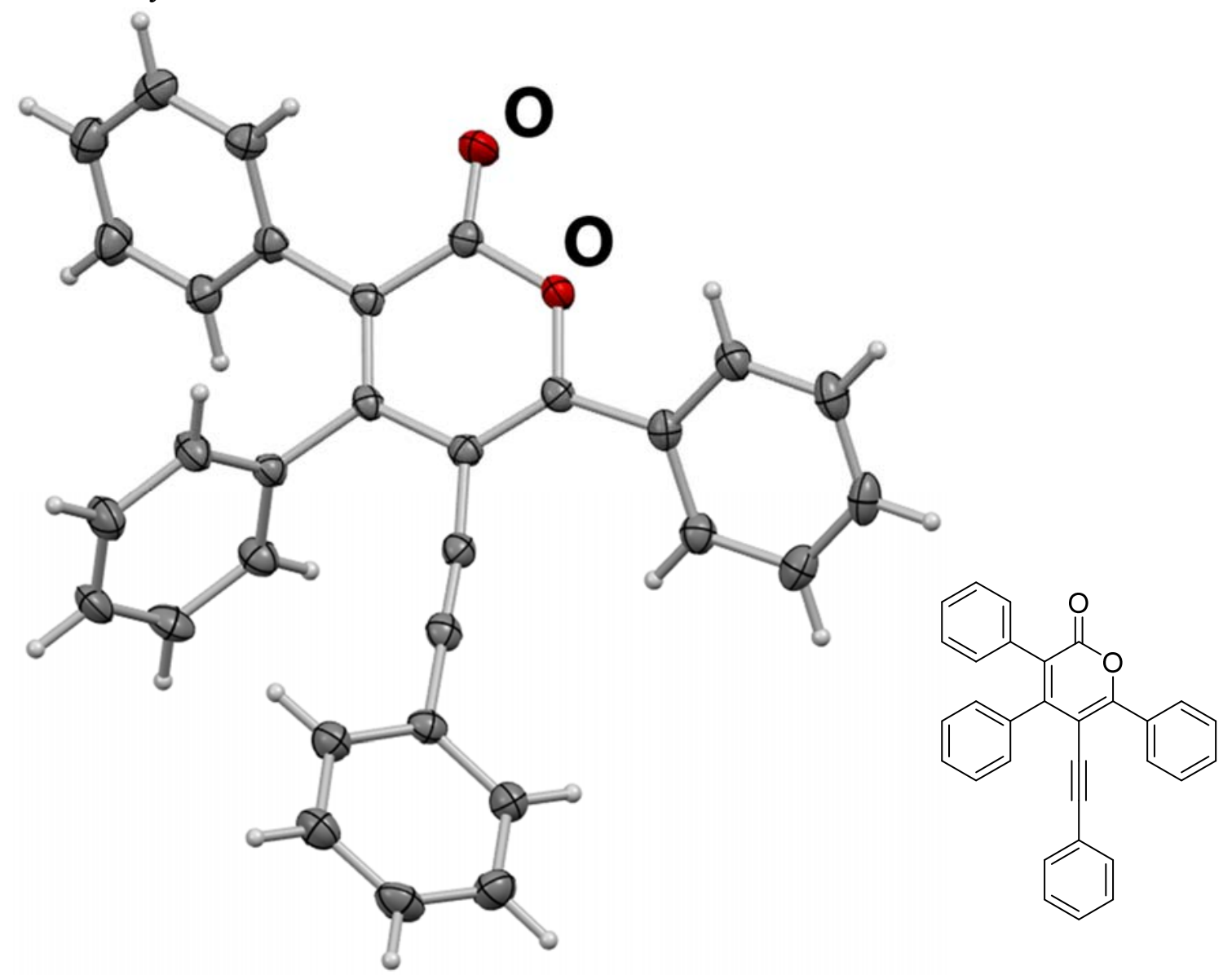

Figure S5. ORTEP drawing of 8 at the $50 \%$ probability level

Table S6. Crystal date and structure solution paramaters of 8

\begin{tabular}{|l|l|l|l|}
\hline Empirical Formula & $\mathrm{C}_{31} \mathrm{H}_{20} \mathrm{O}_{2}$ & Reflections collected & 9535 \\
\hline Formula Weight & 424.47 & Date/restraints/parameters & $4347 / 2 / 298$ \\
\hline Crystal Color, Habit & None & Residuals: $R(I>2.00 \sigma(I))$ & 0.0462 \\
\hline Crystal Dimensions & None & Residuals: $w R(I>2.00 \sigma(I))$ & 0.0894 \\
\hline Crystal System & monoclinic & Goodness of Fit Indicator & 1.056 \\
\hline Lattice Type & Primitive & Flack parameter & $-0.4(9)$ \\
\hline & $a=10.0337(5) \AA$ & Maximum peak in Final Diff. Map & $0.19 \mathrm{e}^{-} / \AA^{3}$ \\
\hline & $b=5.7858(3) \AA$ & Minimum peak in Final Diff. Map & $-0.19 \mathrm{e}^{-} / \AA^{3}$ \\
\hline & $c=19.3039(10) \AA$ & & \\
\hline & $V=1102.10(10) \AA^{3}$ & & \\
\hline & $\beta=100.440(5)^{\circ}$ & & \\
\hline Space Group & $P \mathrm{c}(\# 7)$ & & \\
\hline$Z$ value & 2 & & \\
\hline$D_{\text {calc }}$ & $1.279 \mathrm{~g} / \mathrm{cm}^{3}$ & & \\
\hline$F_{000}$ & 444.00 & & \\
\hline$\mu\left(\mathrm{MoK}_{\alpha}\right)$ & $0.079 \mathrm{~cm}^{-1}$ & & \\
\hline Temperature & $-150.0^{\circ} \mathrm{C}$ & & \\
\hline & \multicolumn{2}{|}{} \\
\hline
\end{tabular}


(2-oxo-4,6-diphenyl-2H-pyran-5-yl)indium diiodide pyridine complex (3b·pyridine) (CCDC 1910738)

3b pyridine was crystallized in chloroform and heptane solvent.

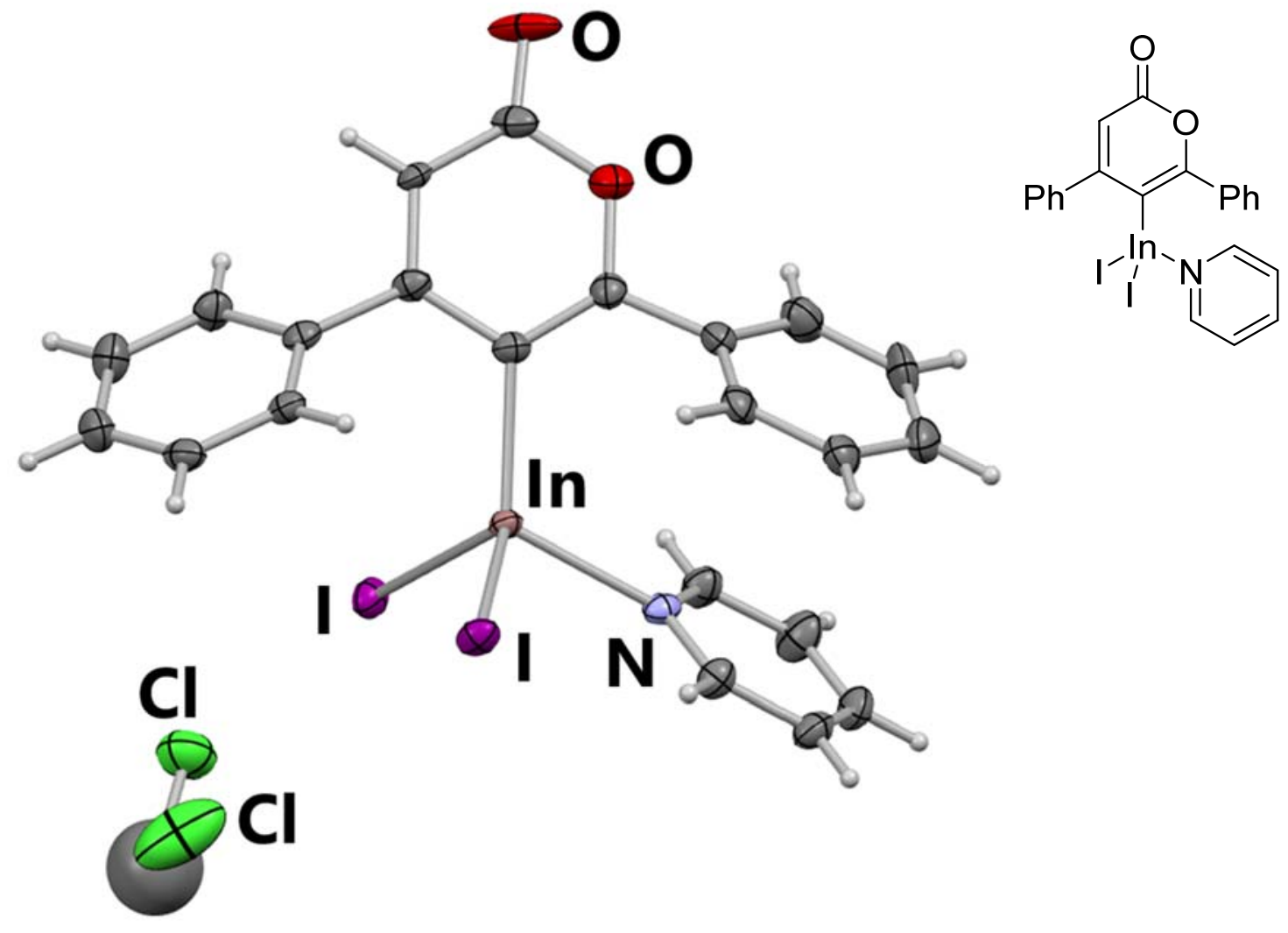

Figure S6. ORTEP drawing of $3 b \cdot$ pyridine at the $50 \%$ probability level

Table S7. Crystal date and structure solution paramaters of $3 \mathbf{b} \cdot$ pyridine

\begin{tabular}{|l|l|l|l|}
\hline Empirical Formula & $\mathrm{C}_{23} \mathrm{H}_{16} \mathrm{Cl}_{3} \mathrm{I}_{2} \mathrm{InNO}_{2}$ & Reflections collected & 33110 \\
\hline Formula Weight & 813.34 & Date/restraints/parameters & $6454 / 0 / 271$ \\
\hline Crystal Color, Habit & None & Residuals: $R(I>2.00 \sigma(I))$ & 0.0331 \\
\hline Crystal Dimensions & None & Residuals: $w R(I>2.00 \sigma(I))$ & 0.1150 \\
\hline Crystal System & Orthorhombic & Goodness of Fit Indicator & 0.892 \\
\hline Lattice Type & Primitive & Maximum peak in Final Diff. Map & $1.04 \mathrm{e}^{-} / \AA^{3}$ \\
\hline & $a=29.4767(8) \AA$ & Minimum peak in Final Diff. Map & $-1.06 \mathrm{e}^{-} / \AA^{3}$ \\
\hline & $b=7.5456(2) \AA$ & & \\
\hline & $c=21.7020(6) \AA$ & & \\
\hline Space Group & $V=4826.9(2) \AA^{3}$ & & \\
\hline$Z$ value & $P b c n(\# 60)$ & & \\
\hline$D_{\text {calc }}$ & 8 & & \\
\hline$F_{000}$ & $2.238 \mathrm{~g} / \mathrm{cm}^{3}$ & & \\
\hline$\mu($ MoK $\alpha)$ & 3064.00 & & \\
\hline Temperature & $3.892 \mathrm{~cm}^{-1}$ & & \\
\hline
\end{tabular}


zwitterion intermediate $\mathbf{4 b}$ (CCDC 1910563)

4b was recrystallized in chloroform solvent.

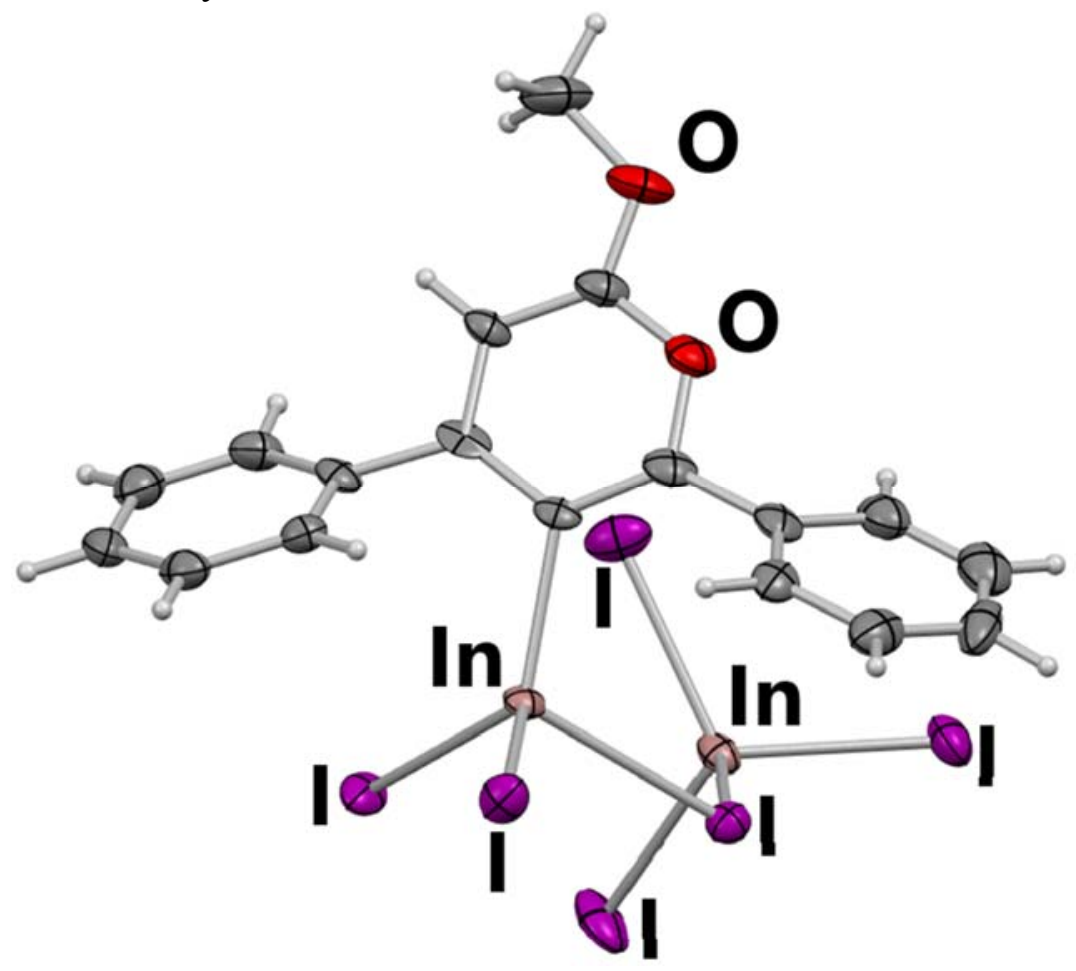<smiles></smiles>

Figure S7. ORTEP drawing of $4 \mathrm{~b}$ at the $50 \%$ probability level

Table S8. Crystal date and structure solution paramaters of $\mathbf{4 b}$

\begin{tabular}{|l|l|l|l|}
\hline Empirical Formula & $\mathrm{C}_{18} \mathrm{H}_{14} \mathrm{I}_{6} \mathrm{In}_{2} \mathrm{O}_{2}$ & Function Minimized & $\Sigma \mathrm{w}(|\mathrm{Fo}|-|\mathrm{Fc}|)^{2}$ \\
\hline Formula Weight & 1253.37 & Least Squares Weights & $1 / 2(\mathrm{Fo})$ \\
\hline Crystal Color, Habit & None & No. Observations $(I>2.00 \sigma(I))$ & 5338 \\
\hline Crystal Dimensions & None & No. Variables & 267 \\
\hline Crystal System & Orthorhombic & Reflection/Parameter Ratio & 19.99 \\
\hline Lattice Type & Primitive & Residuals: $R(I>2.00 \sigma(I))$ & 0.0504 \\
\hline & $a=14.25000 \AA$ & Residuals: $w R(I>2.00 \sigma(I))$ & 0.0546 \\
\hline & $b=14.31400 \AA$ & Goodness of Fit Indicator & 2.027 \\
\hline & $c=28.24020 \AA$ & Max Shift/Error in Final Cycle & 0.000 \\
\hline & $V=5760.28067 \AA^{3}$ & Maximum peak in Final Diff. Map & $1.85 \mathrm{e}^{-} / \AA^{3}$ \\
\hline Space Group & $P b c a(\# 61)$ & Minimum peak in Final Diff. Map & $-1.79 \mathrm{e}^{-} / \AA^{3}$ \\
\hline$Z$ value & 8 & & \\
\hline$D_{\text {calc }}$ & $2.890 \mathrm{~g} / \mathrm{cm}^{3}$ & & \\
\hline$F_{000}$ & 4432.00 & & \\
\hline$\mu\left(M_{0 K}\right)$ & $633.773 \mathrm{~cm}^{-1}$ & & \\
\hline Temperature & $-150.0^{\circ} \mathrm{C}$ & & \\
\hline
\end{tabular}




\section{NMR spectra}

methyl (Z)-5-phenylpent-2-en-4-ynoate (1a)<smiles>COC(=O)/C=C/c1ccccc1</smiles>

${ }^{1} \mathrm{H} \mathrm{NMR}\left(400 \mathrm{MHz}, \mathrm{CDCl}_{3}\right)$

மำ

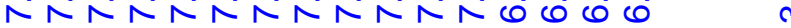
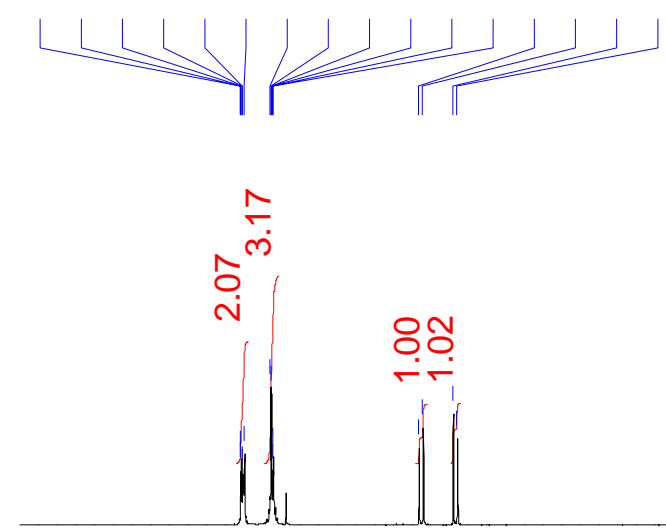

$\stackrel{0}{m}$

PPM

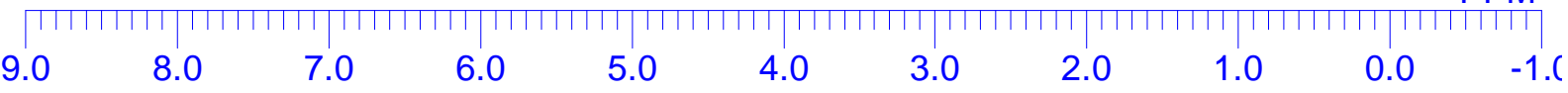

${ }^{13} \mathrm{C}\left\{{ }^{1} \mathrm{H}\right\}$ NMR $\left(100 \mathrm{MHz}, \mathrm{CDCl}_{3}\right)$

$\underset{N}{N}$

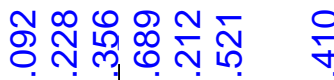

กิ่

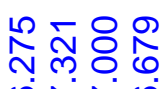

穴令

ำ
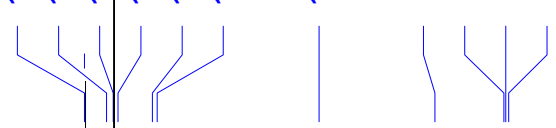

เก 
methyl (Z)-3,5-diphenylpent-2-en-4-ynoate (1b)<smiles>COC(=O)/C=C(\C#Cc1ccccc1)c1ccccc1</smiles>

${ }^{1} \mathrm{H}$ NMR (400 MHz, $\mathrm{CDCl}_{3}$ )

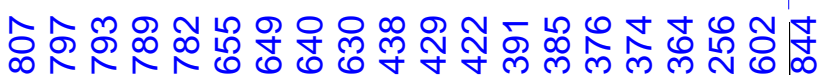

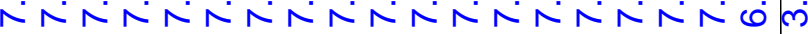

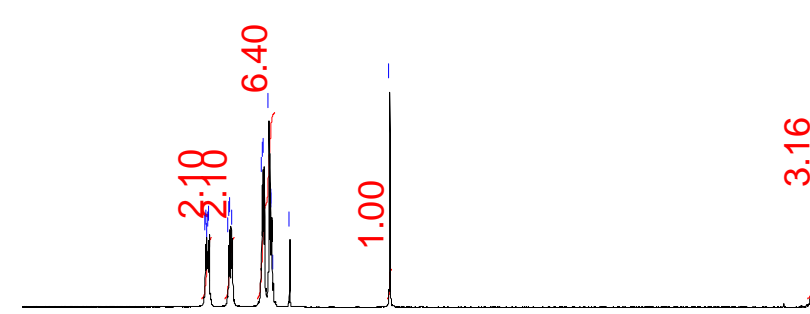

$\stackrel{2}{\rightarrow}$

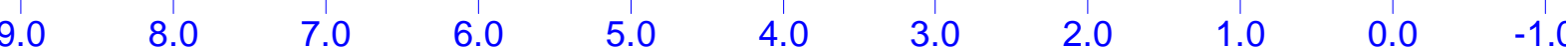

${ }^{13} \mathrm{C}\left\{{ }^{1} \mathrm{H}\right\} \mathrm{NMR}\left(100 \mathrm{MHz}, \mathrm{CDCl}_{3}\right)$

@

பி

규용

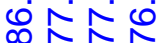

$\stackrel{\substack{\infty \\ \infty}}{\infty}$

ન

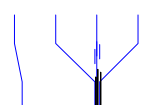

เก

PPM

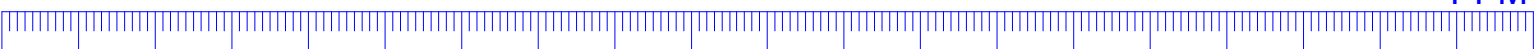

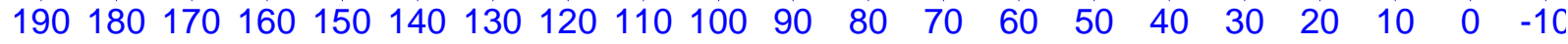


methyl (Z)-2-ethyl-3,5-diphenylpent-2-en-4-ynoate (1c)<smiles>CCC(C(=O)OC)=C(C#Cc1ccccc1)c1ccccc1</smiles>

${ }^{1} \mathrm{H}$ NMR (400 MHz, $\left.\mathrm{CDCl}_{3}\right)$

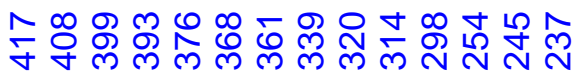

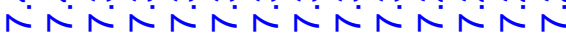
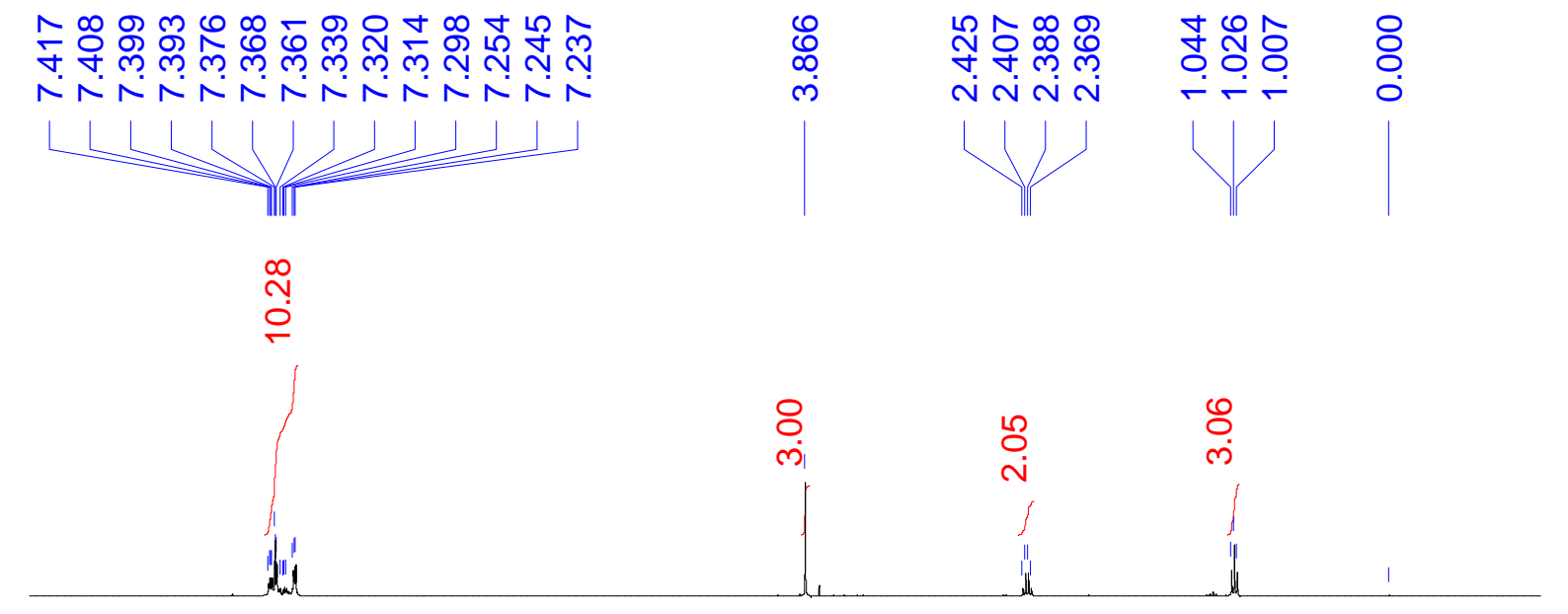

PPM

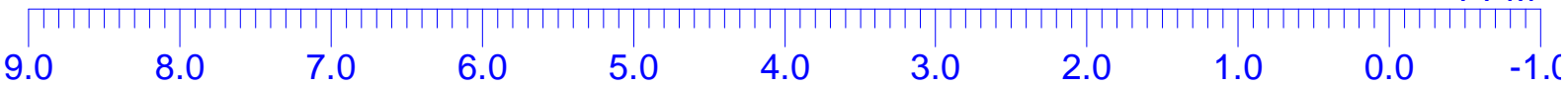

${ }^{13} \mathrm{C}\left\{{ }^{1} \mathrm{H}\right\}$ NMR $\left(100 \mathrm{MHz}, \mathrm{CDCl}_{3}\right)$

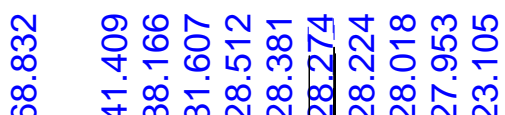

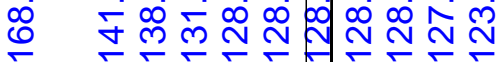

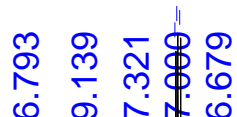

の

ָָ

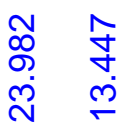
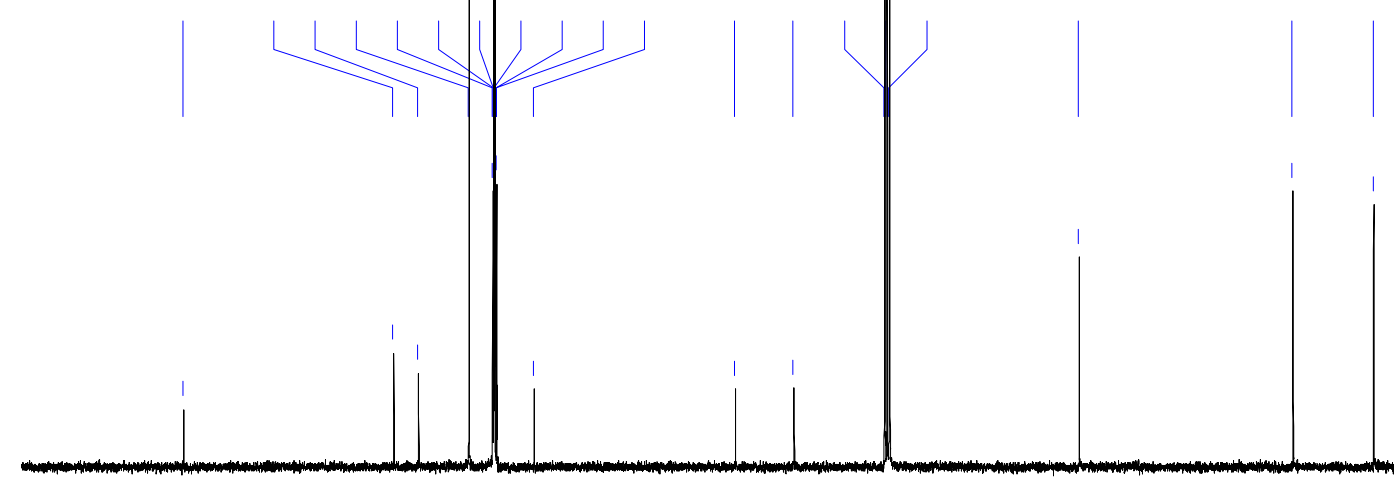

PPM

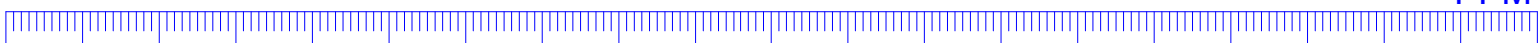

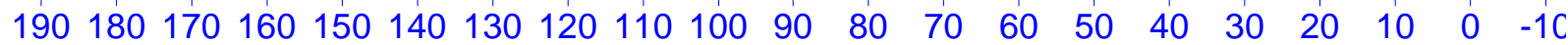


methyl (Z)-5-(p-tolyl)pent-2-en-4-ynoate (1d)<smiles>COC(=O)/C=C\C#Cc1ccc(C)cc1</smiles>

${ }^{1} \mathrm{H}$ NMR (400 MHz, $\mathrm{CDCl}_{3}$ )

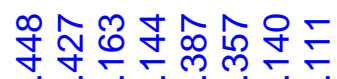

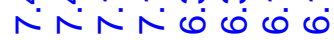

HW

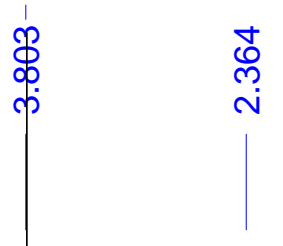

8

ํํ유 운

$\stackrel{10}{\rightarrow}$ ले
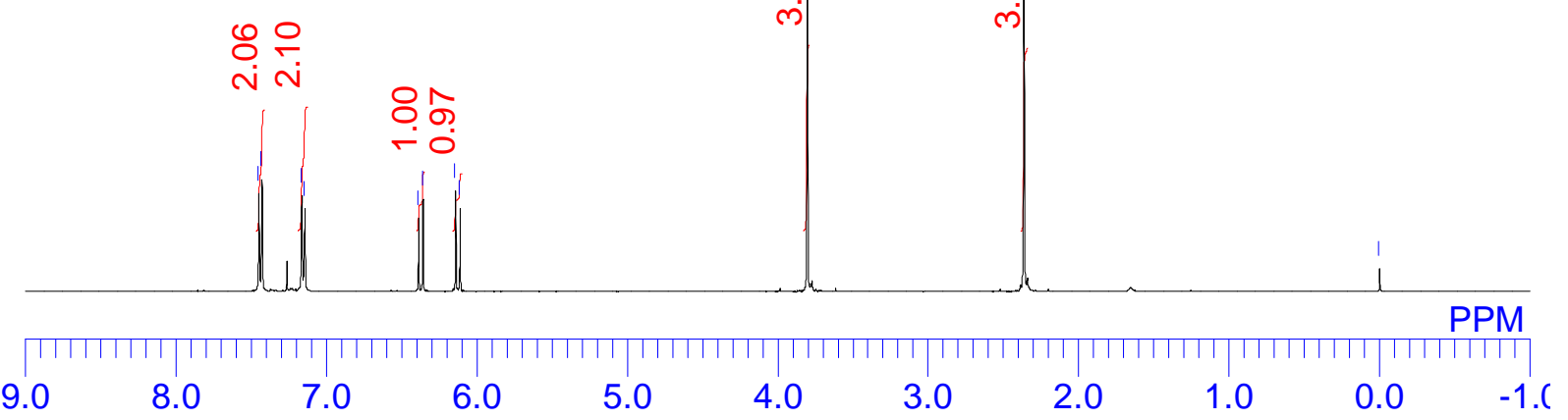

${ }^{13} \mathrm{C}\left\{{ }^{1} \mathrm{H}\right\}$ NMR $\left(100 \mathrm{MHz}, \mathrm{CDCl}_{3}\right)$

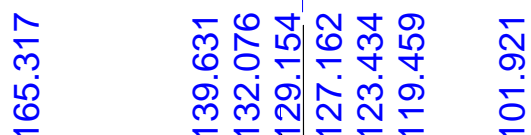

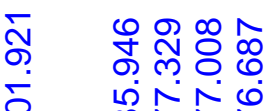

亦令

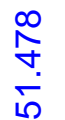

占
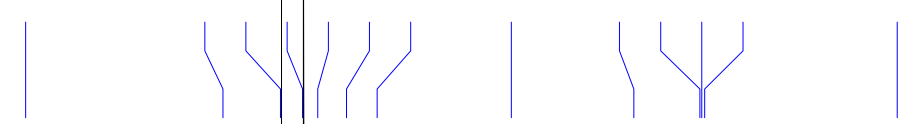

PPM

|

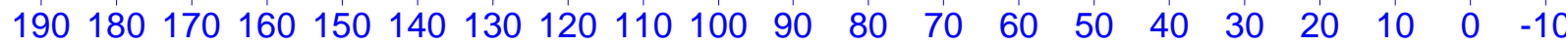


methyl (Z)-5-(4-(tert-butyl)phenyl)pent-2-en-4-ynoate (1e)<smiles>COC(=O)/C=C\C#Cc1ccc(C(C)(C)C)cc1</smiles>

${ }^{1} \mathrm{H} \mathrm{NMR}\left(400 \mathrm{MHz}, \mathrm{CDCl}_{3}\right)$

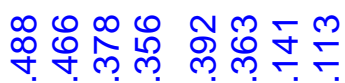
ベペ
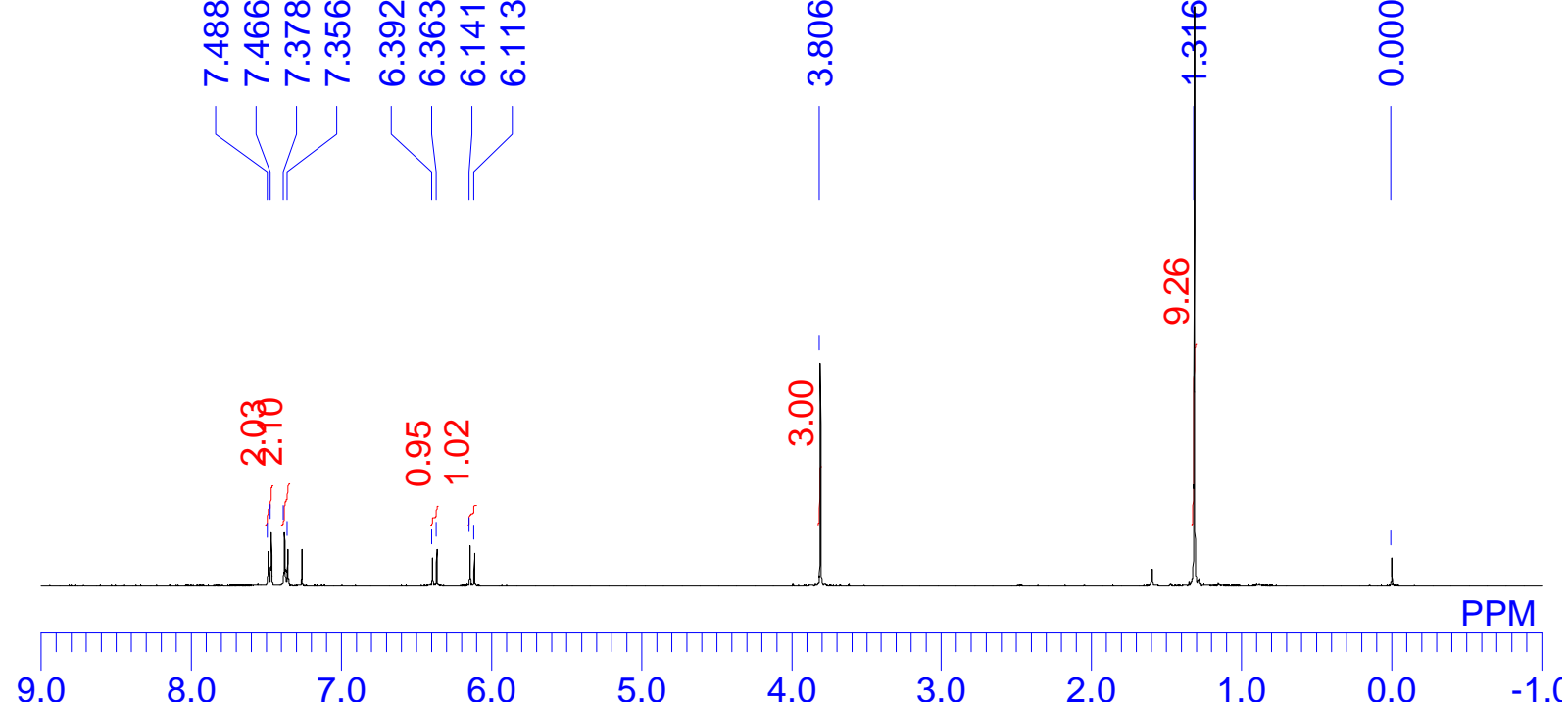

${ }^{13} \mathrm{C}\left\{{ }^{1} \mathrm{H}\right\}$ NMR $\left(100 \mathrm{MHz}, \mathrm{CDCl}_{3}\right)$

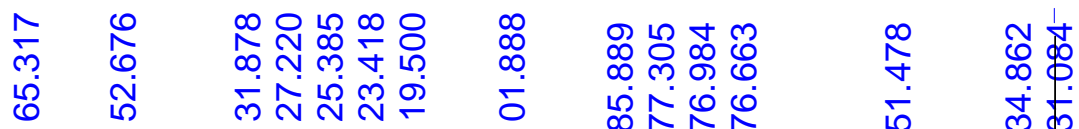

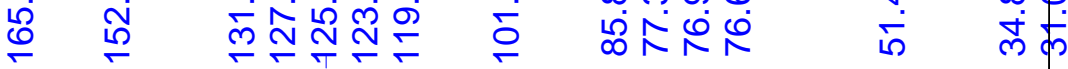

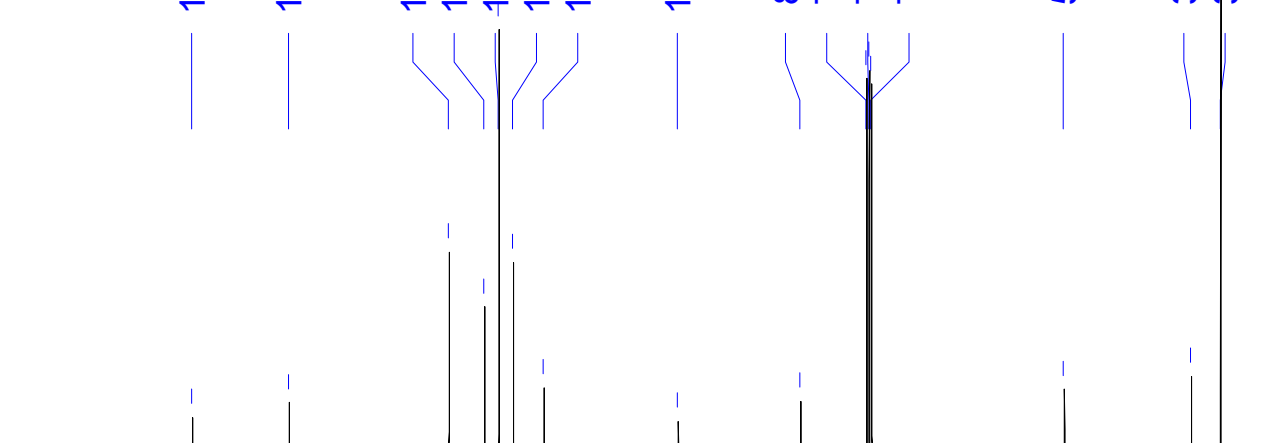


methyl (Z)-5-(4-methoxyphenyl)pent-2-en-4-ynoate (1f)<smiles>COC(=O)/C=C\C#Cc1ccc(OC)cc1</smiles>

${ }^{1} \mathrm{H} \mathrm{NMR}\left(400 \mathrm{MHz}, \mathrm{CDCl}_{3}\right)$

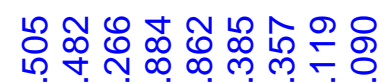
ベペ்
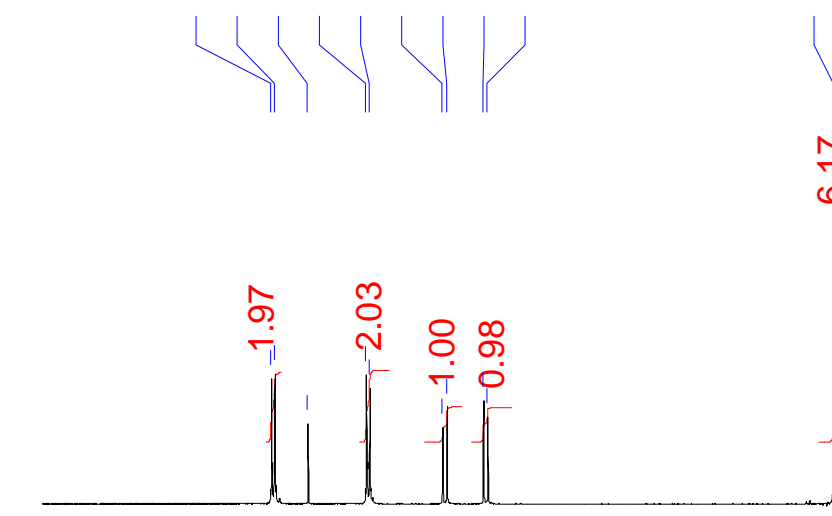

PPM

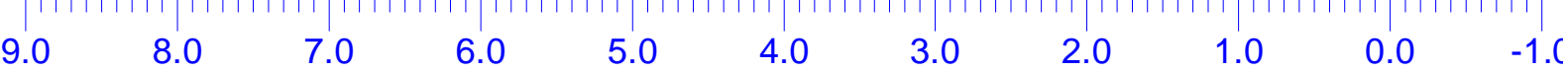

${ }^{13} \mathrm{C}\left\{{ }^{1} \mathrm{H}\right\}$ NMR $\left(100 \mathrm{MHz}, \mathrm{CDCl}_{3}\right)$

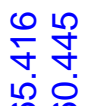

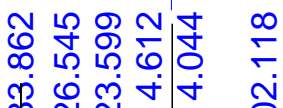

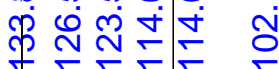

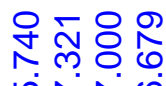

经走定

\section{నิ เ্ণ}

เึ่
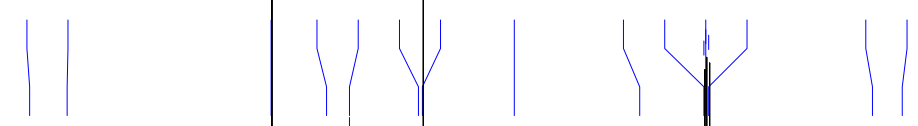

PPM

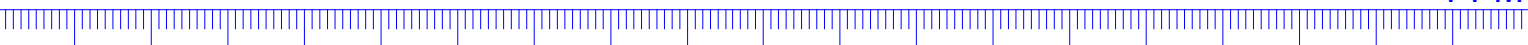

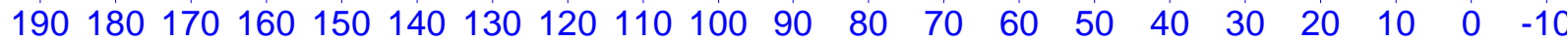


methyl (Z)-5-(4-chlorophenyl)pent-2-en-4-ynoate (1g)<smiles>COC(=O)/C=C\C#Cc1ccc(Cl)cc1</smiles>

${ }^{1} \mathrm{H} \mathrm{NMR}\left(400 \mathrm{MHz}, \mathrm{CDCl}_{3}\right)$

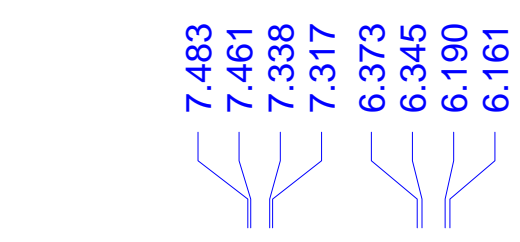

1
$\substack{\infty \\ \infty \\ m}$

8

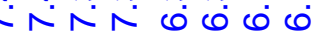
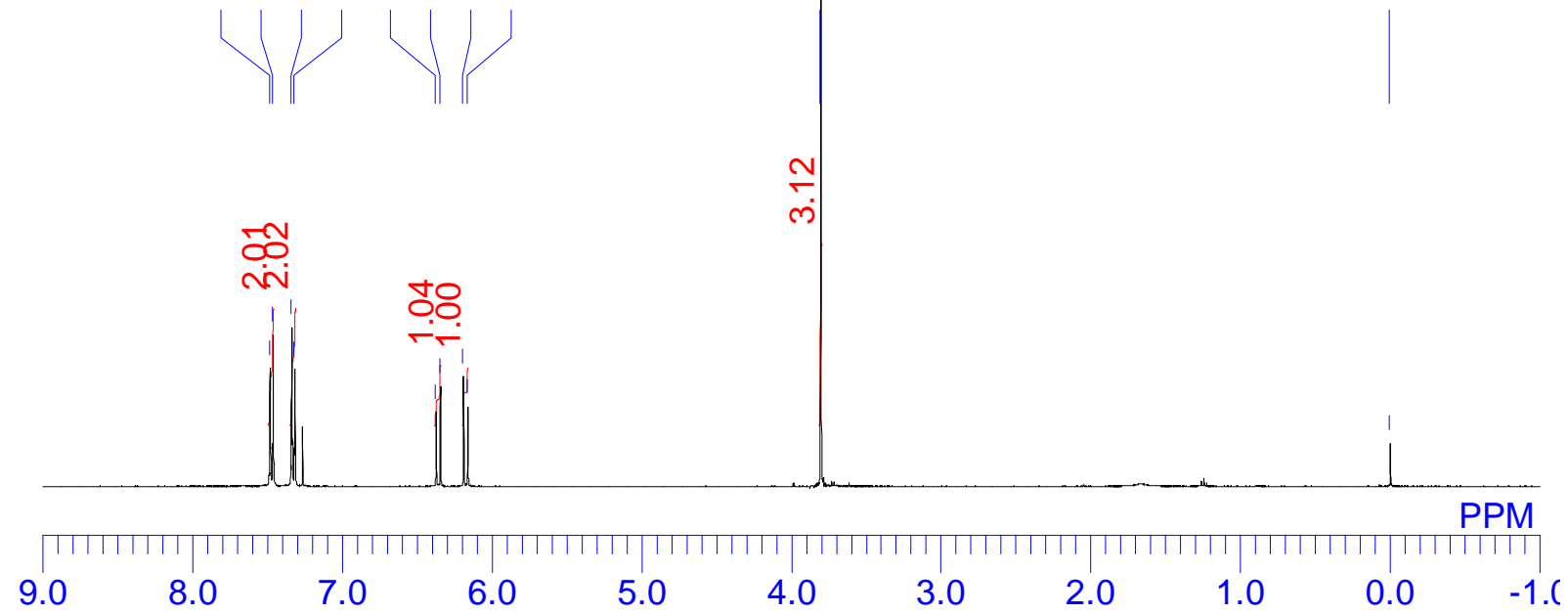

${ }^{13} \mathrm{C}\left\{{ }^{1} \mathrm{H}\right\}$ NMR $\left(100 \mathrm{MHz}, \mathrm{CDCl}_{3}\right)$

疍

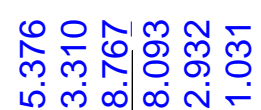

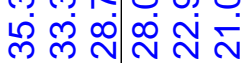

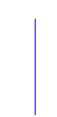


methyl (Z)-5-(3-fluorophenyl)pent-2-en-4-ynoate (1h)<smiles>COC(=O)/C=C\C#Cc1cccc(F)c1</smiles>

${ }^{1} \mathrm{H} \mathrm{NMR}\left(400 \mathrm{MHz}, \mathrm{CDCl}_{3}\right)$
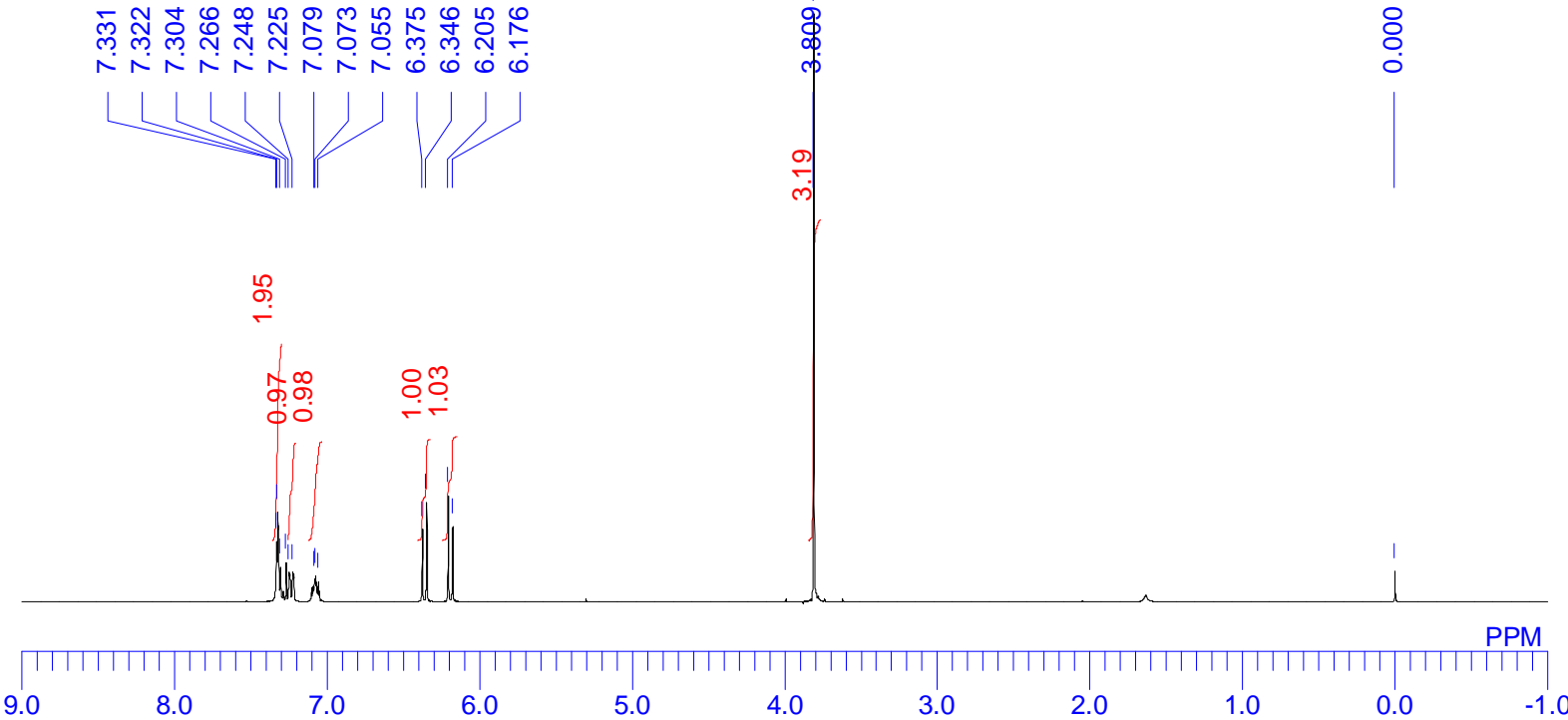

${ }^{13} \mathrm{C}\left\{{ }^{1} \mathrm{H}\right\}$ NMR $\left(100 \mathrm{MHz}, \mathrm{CDCl}_{3}\right)$

옹ㅇำ

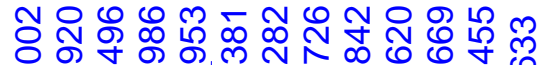

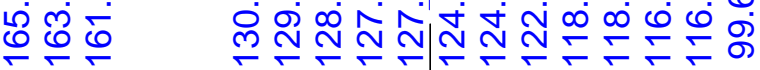

సิ

め

$\infty_{\infty}^{\infty}$

เก

II
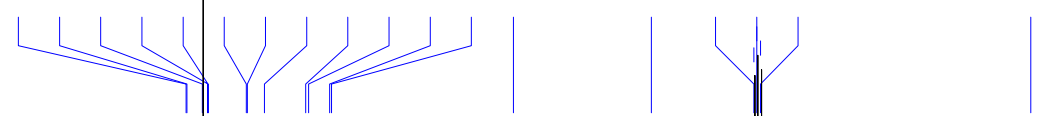

PPM

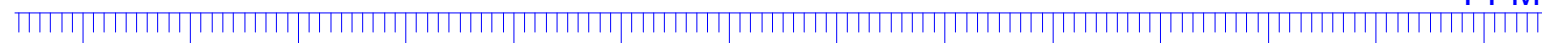

$\begin{array}{llllllllllllll}170 & 160 & 150 & 140 & 130 & 120 & 110 & 100 & 90 & 80 & 70 & 60 & 50 & 40\end{array}$ 
methyl (Z)-undec-2-en-4-ynoate (1i)

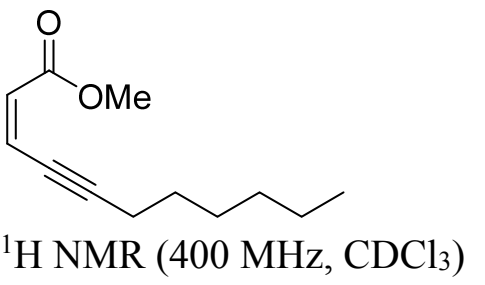

恣 ‥
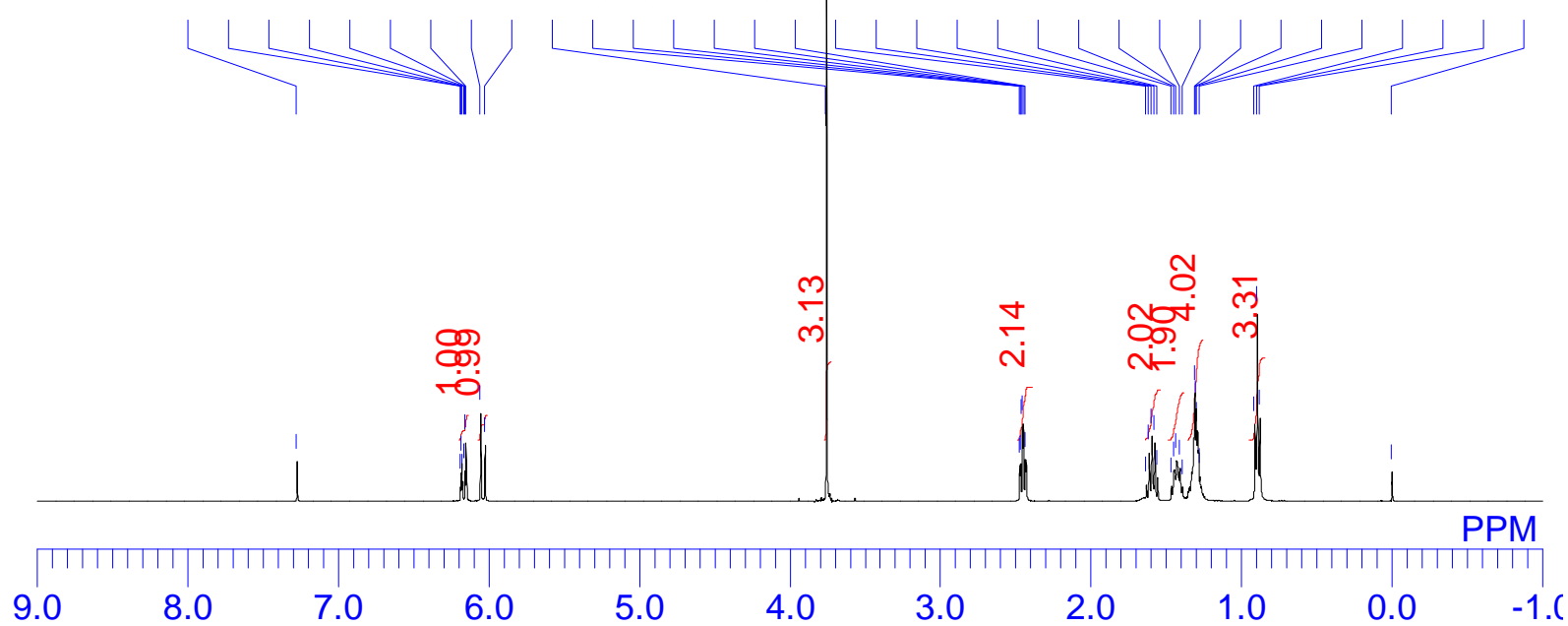

${ }^{13} \mathrm{C}\left\{{ }^{1} \mathrm{H}\right\}$ NMR $\left(100 \mathrm{MHz}, \mathrm{CDCl}_{3}\right)$

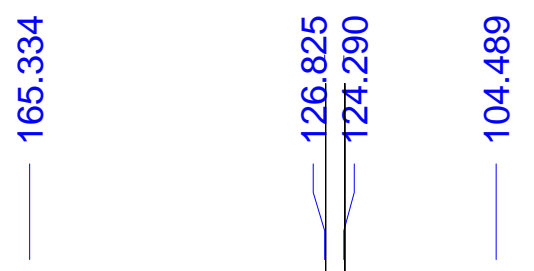

PPM

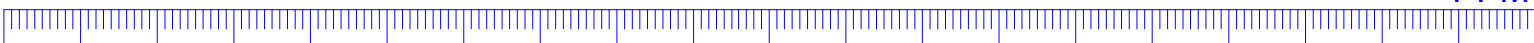

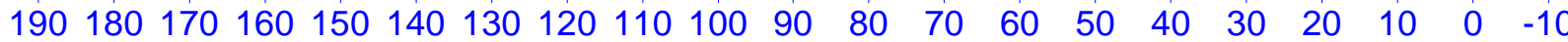


methyl (Z)-5-cyclopropylpent-2-en-4-ynoate (1j)<smiles>COC(=O)/C=C\C#CC1CC1</smiles>

${ }^{1} \mathrm{H} \mathrm{NMR}\left(400 \mathrm{MHz}, \mathrm{CDCl}_{3}\right)$

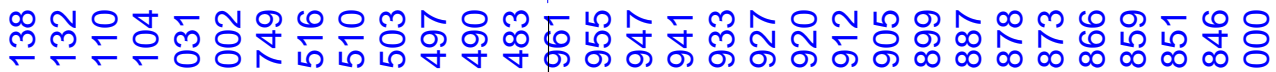
ڤं
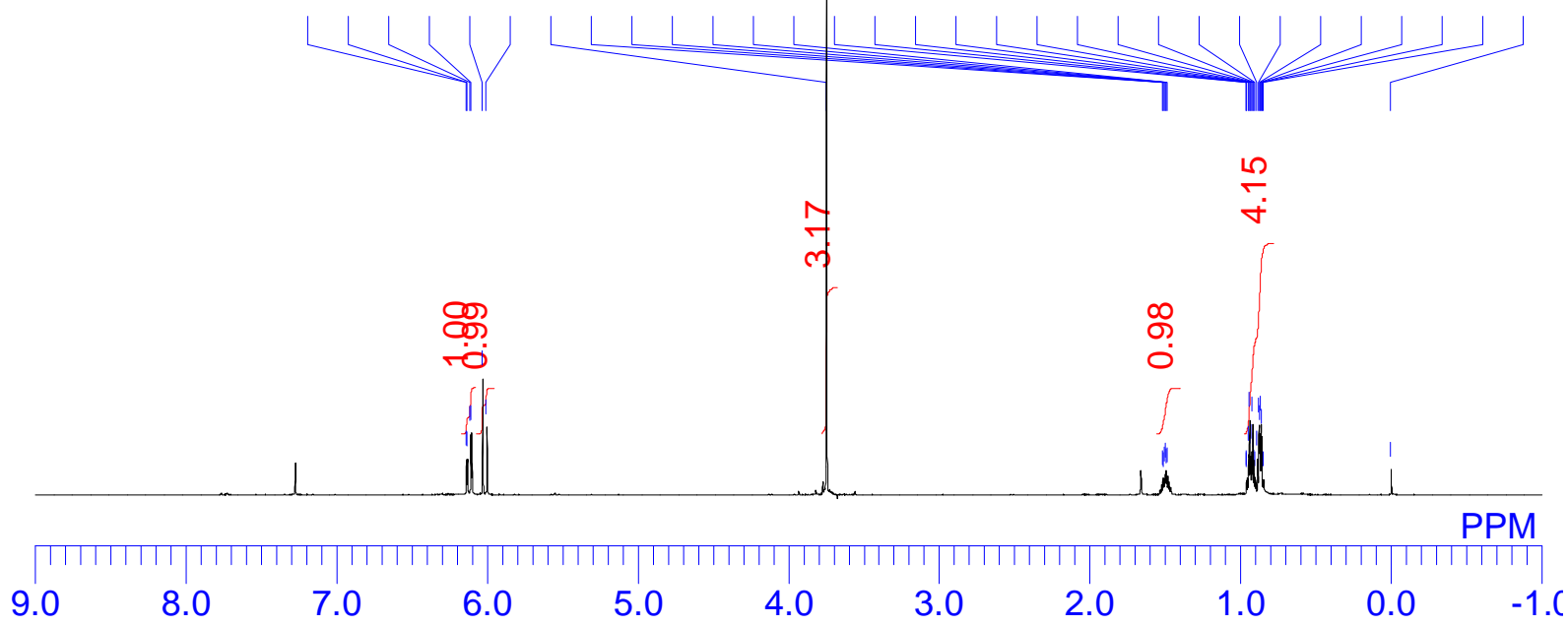

${ }^{13} \mathrm{C}\left\{{ }^{1} \mathrm{H}\right\}$ NMR $\left(100 \mathrm{MHz}, \mathrm{CDCl}_{3}\right)$

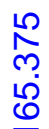

踏

$\checkmark$ m

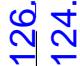

돔
엄
엄

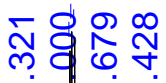

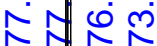

ڤิ

$\begin{array}{ll}m & 0 \\ \text { जै } & \infty \\ \text { बे } & 0\end{array}$
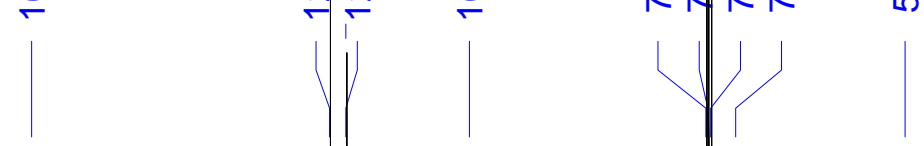

PPM

|

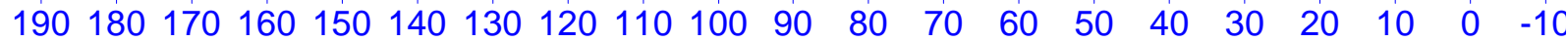


dimethyl 2-(3-phenylprop-2-yn-1-ylidene)malonate (1k)<smiles>COC(=O)C(=CC#Cc1ccccc1)C(=O)OC</smiles>

${ }^{1} \mathrm{H} \mathrm{NMR}\left(400 \mathrm{MHz}, \mathrm{CDCl}_{3}\right)$

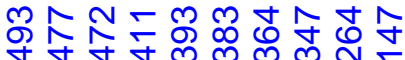

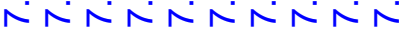

సิ

ঃ

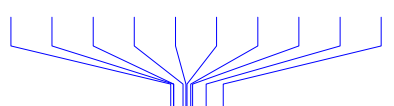

$m$ m
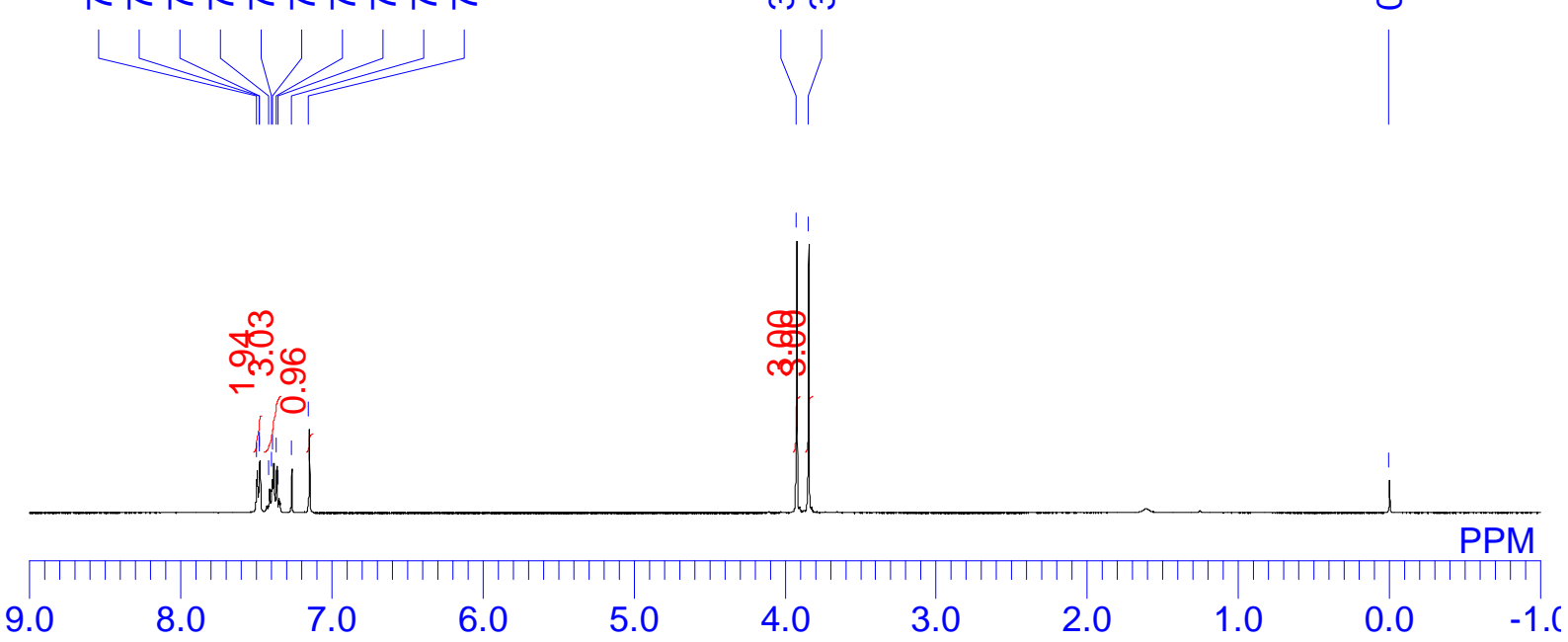

${ }^{13} \mathrm{C}\left\{{ }^{1} \mathrm{H}\right\} \mathrm{NMR}\left(100 \mathrm{MHz}, \mathrm{CDCl}_{3}\right)$

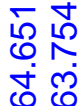

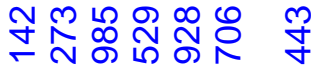

듀용

ก

它

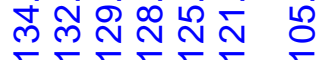

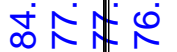

กิ่
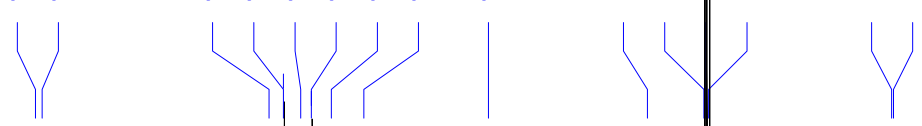

PPM

ता

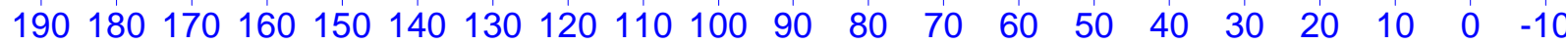


methyl (Z)-5-cyclopropyl-2-ethyl-3-phenylpent-2-en-4-ynoate (1I)<smiles>CC/C(C(=O)OC)=C(/C#CC1CC1)c1ccccc1</smiles>

${ }^{1} \mathrm{H}$ NMR (400 MHz, $\left.\mathrm{CDCl}_{3}\right)$

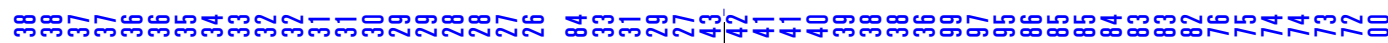

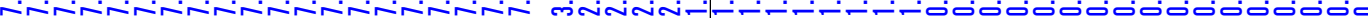
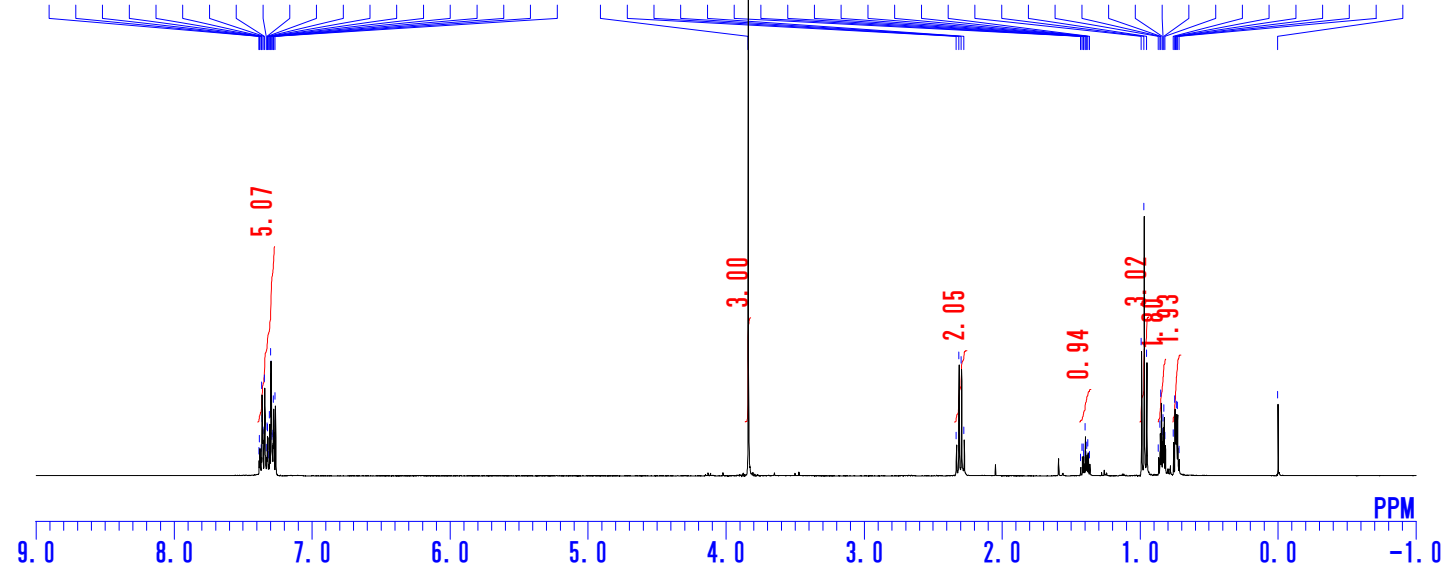

${ }^{13} \mathrm{C}\left\{{ }^{1} \mathrm{H}\right\} \mathrm{NMR}\left(100 \mathrm{MHz}, \mathrm{CDCl}_{3}\right)$

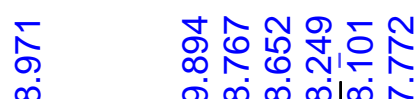

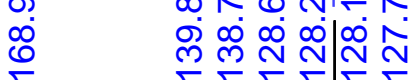

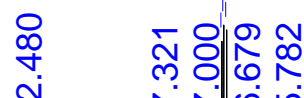

ペ余官

ชิ

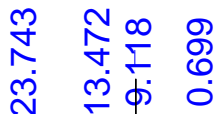

$\mid$

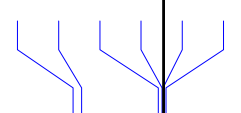

응
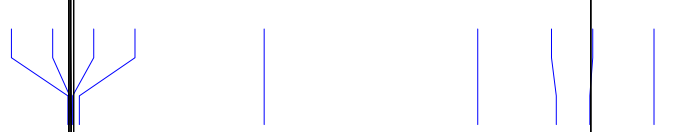

PPM

|

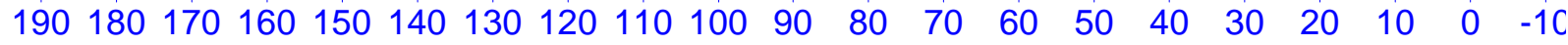


methyl (Z)-2,3,5-triphenylpent-2-en-4-ynoate (1m)<smiles>COC(=O)C(C#Cc1ccccc1)=C(c1ccccc1)c1ccccc1</smiles>

${ }^{1} \mathrm{H} \mathrm{NMR}\left(400 \mathrm{MHz}, \mathrm{CDCl}_{3}\right)$
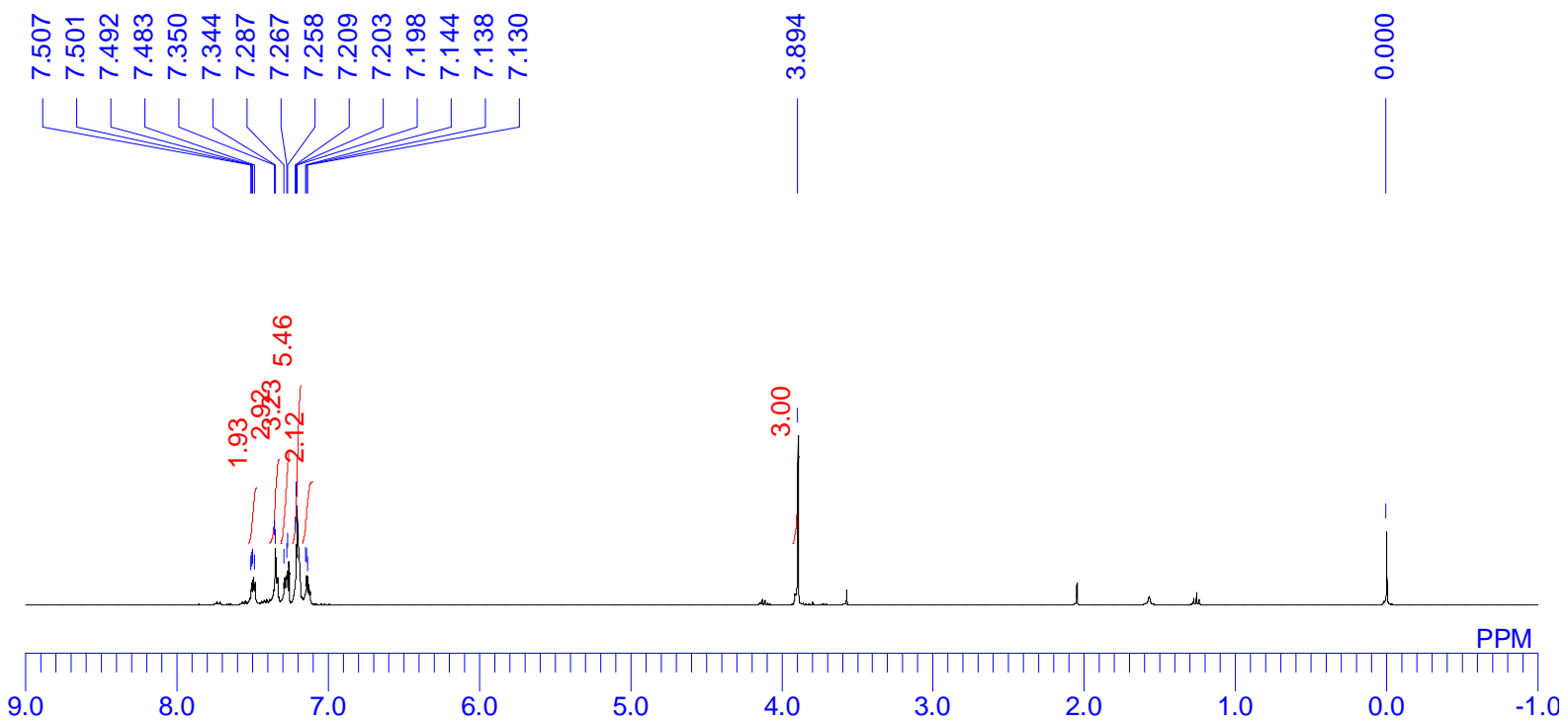

${ }^{13} \mathrm{C}\left\{{ }^{1} \mathrm{H}\right\}$ NMR $\left(100 \mathrm{MHz}, \mathrm{CDCl}_{3}\right)$

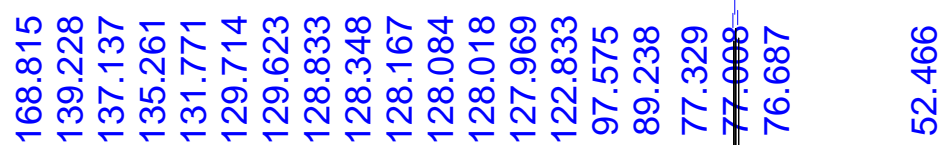
m $m$ m N N N N N N N
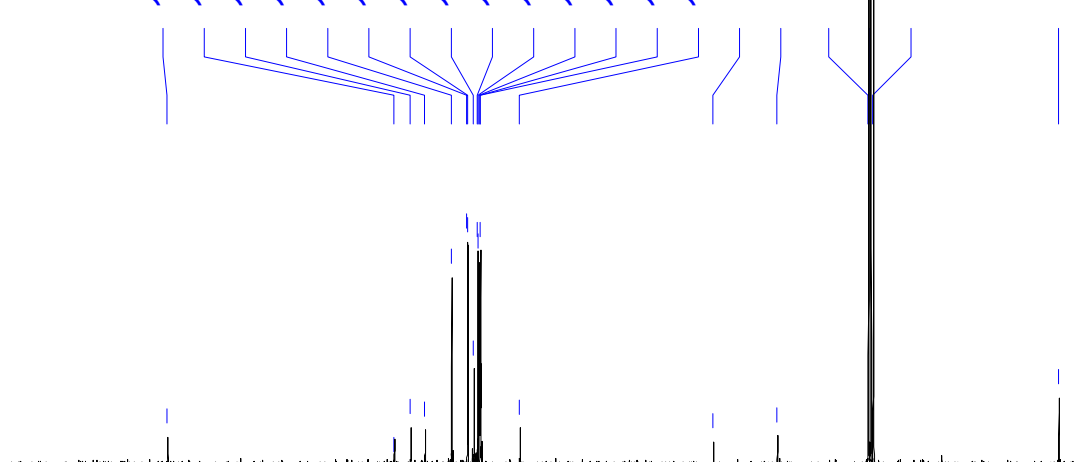
6-phenyl-2H-pyran-2-one (2a)<smiles>O=c1cccc(-c2ccccc2)o1</smiles>

${ }^{1} \mathrm{H} \mathrm{NMR}\left(400 \mathrm{MHz}, \mathrm{CDCl}_{3}\right)$

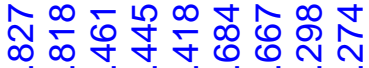

৪ ヘヘヘペ்

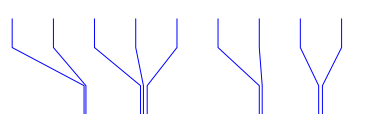

$\stackrel{+}{\circ}$

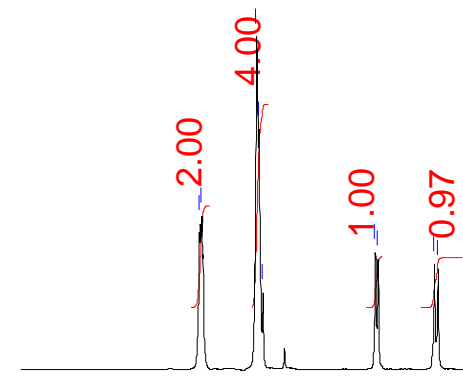

PPM

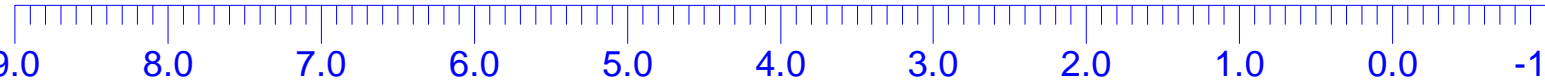

${ }^{13} \mathrm{C}\left\{{ }^{1} \mathrm{H}\right\} \mathrm{NMR}\left(100 \mathrm{MHz}, \mathrm{CDCl}_{3}\right)$

욜

年

它完 竎

Nิ용
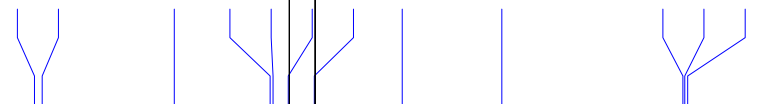

PPM

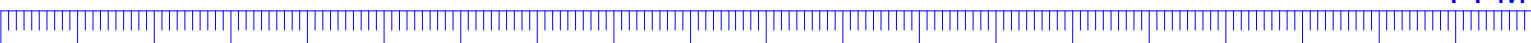

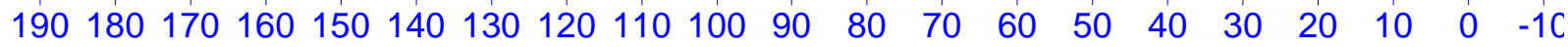


4,6-diphenyl-2H-pyran-2-one (2b)<smiles>O=c1cc(-c2ccccc2)cc(-c2ccccc2)o1</smiles>

${ }^{1} \mathrm{H} \mathrm{NMR}\left(400 \mathrm{MHz}, \mathrm{CDCl}_{3}\right)$

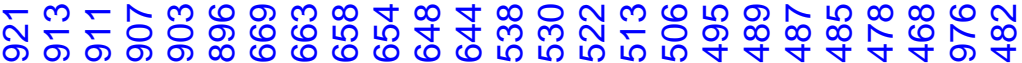

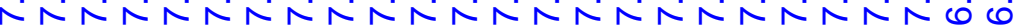
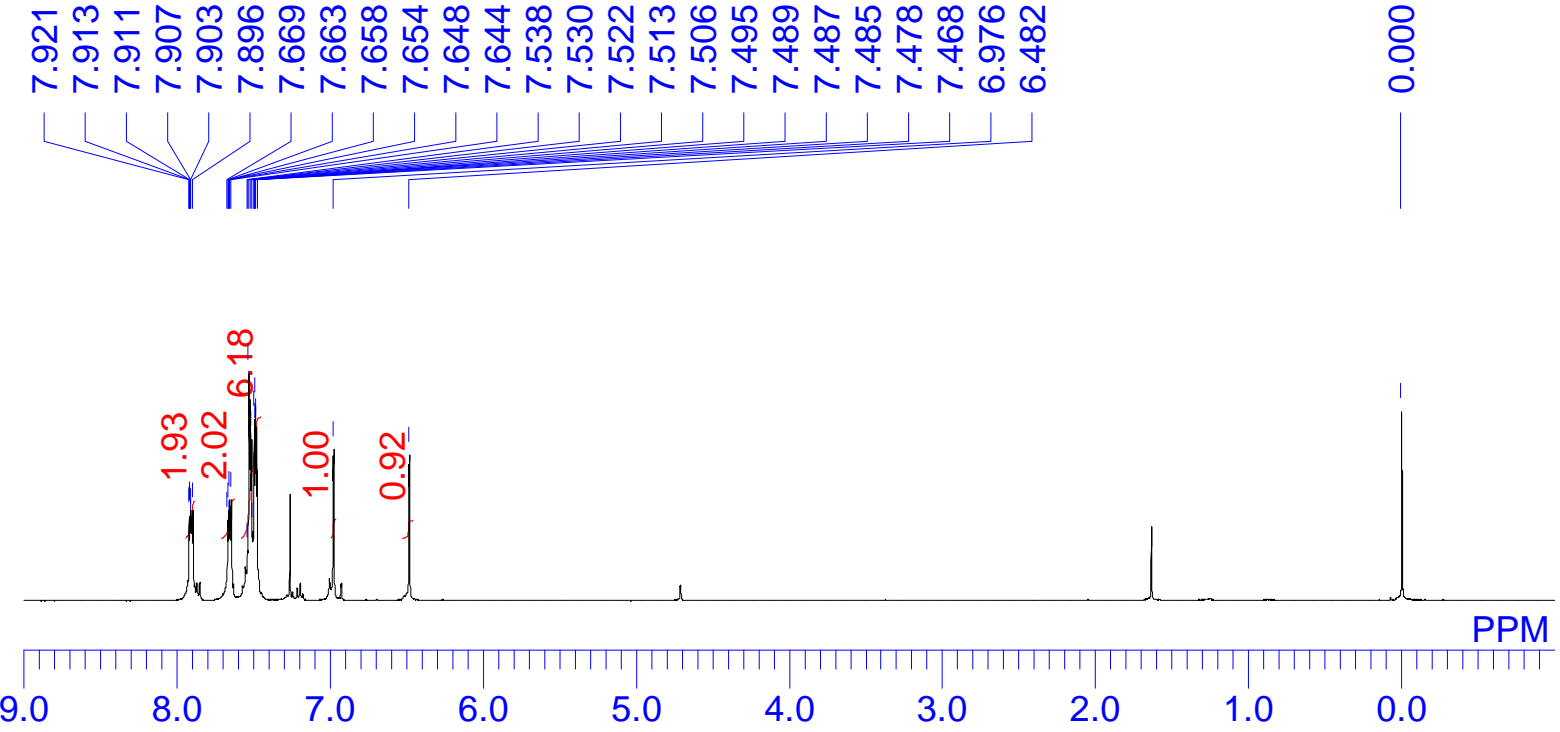

${ }^{13} \mathrm{C}\left\{{ }^{1} \mathrm{H}\right\} \mathrm{NMR}\left(100 \mathrm{MHz}, \mathrm{CDCl}_{3}\right)$

mొ

过

กำ

Nंก்
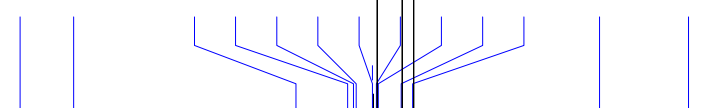

ヘト

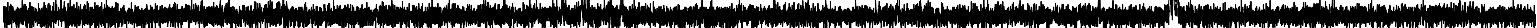

PPM

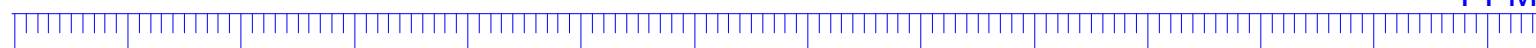

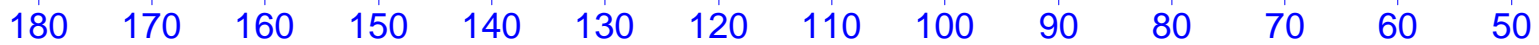


3-ethyl-4,6-diphenyl-2H-pyran-2-one (2c)<smiles>CCc1c(-c2ccccc2)cc(-c2ccccc2)oc1=O</smiles>

${ }^{1} \mathrm{H} \mathrm{NMR}\left(400 \mathrm{MHz}, \mathrm{CDCl}_{3}\right)$

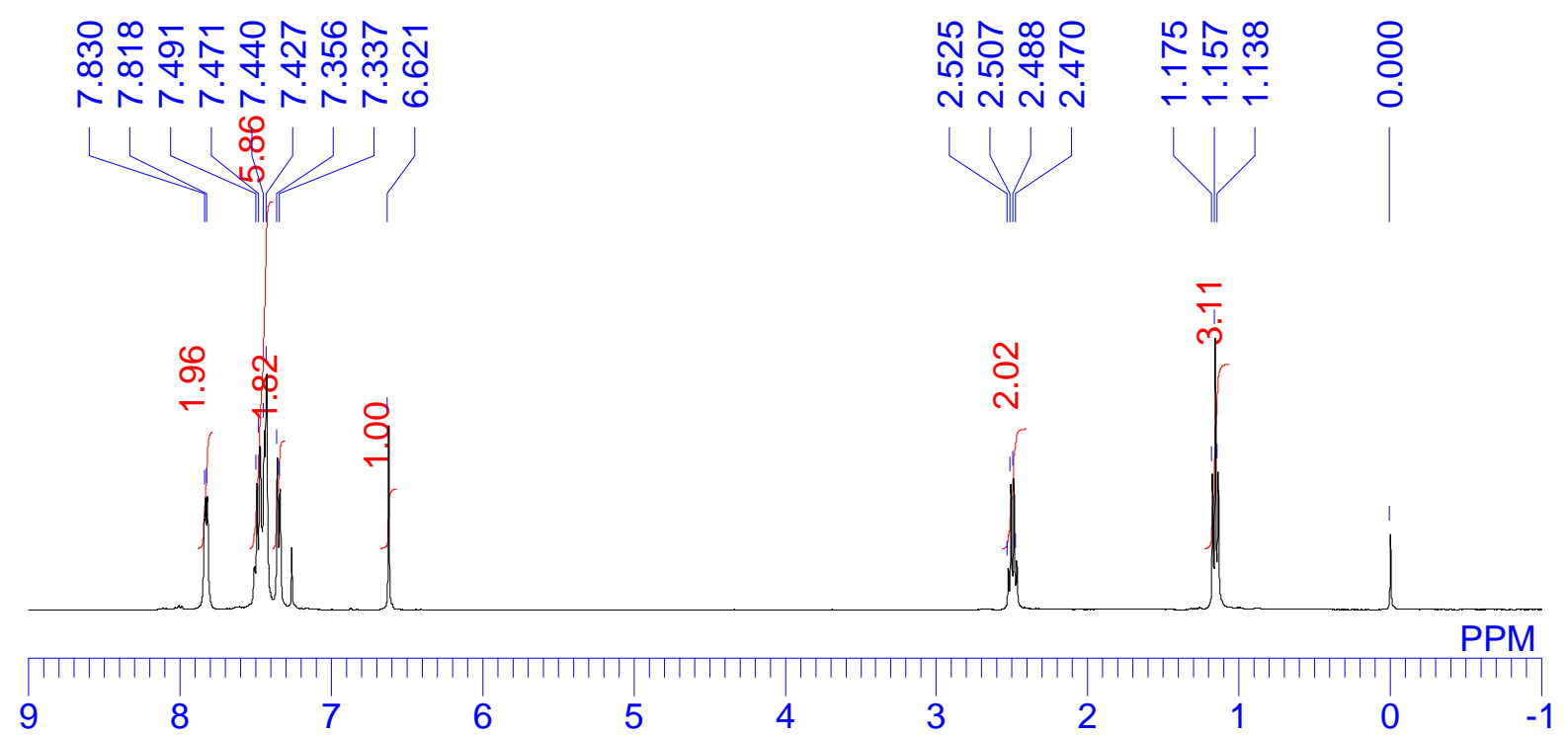

${ }^{13} \mathrm{C}\left\{{ }^{1} \mathrm{H}\right\}$ NMR $\left(100 \mathrm{MHz}, \mathrm{CDCl}_{3}\right)$

두ㅇㅝㅠ

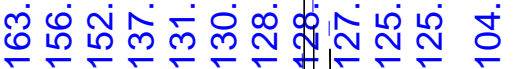

तี่

సं。

$\stackrel{0}{i}$ 워
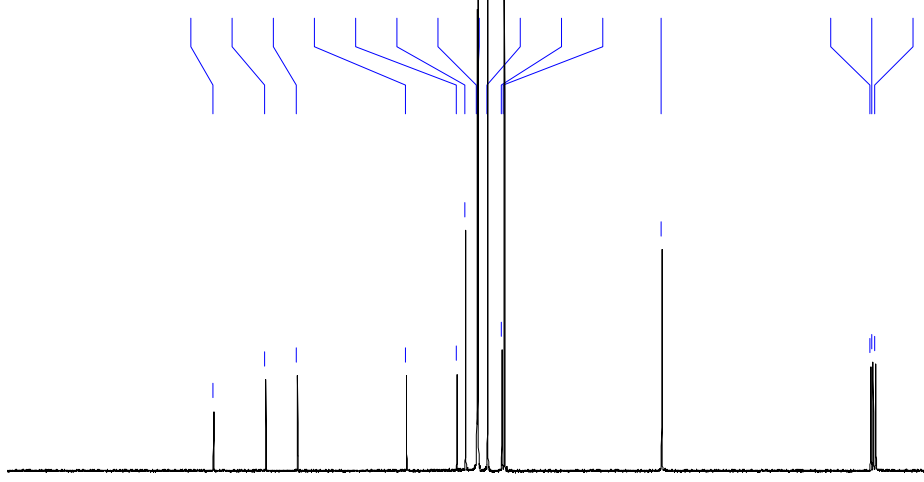

ते

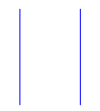

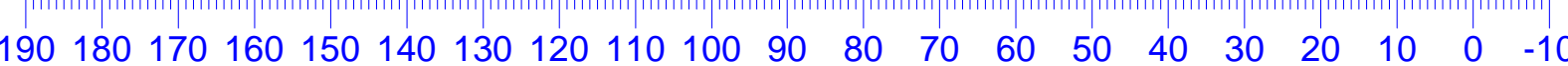


5-iodo-6-phenyl-2H-pyran-2-one (5a)<smiles>O=c1ccc(I)c(-c2ccccc2)o1</smiles>

${ }^{1} \mathrm{H} \mathrm{NMR}\left(400 \mathrm{MHz}, \mathrm{CDCl}_{3}\right)$

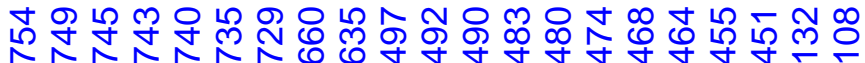

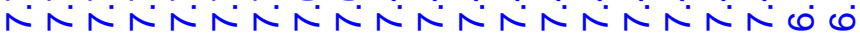
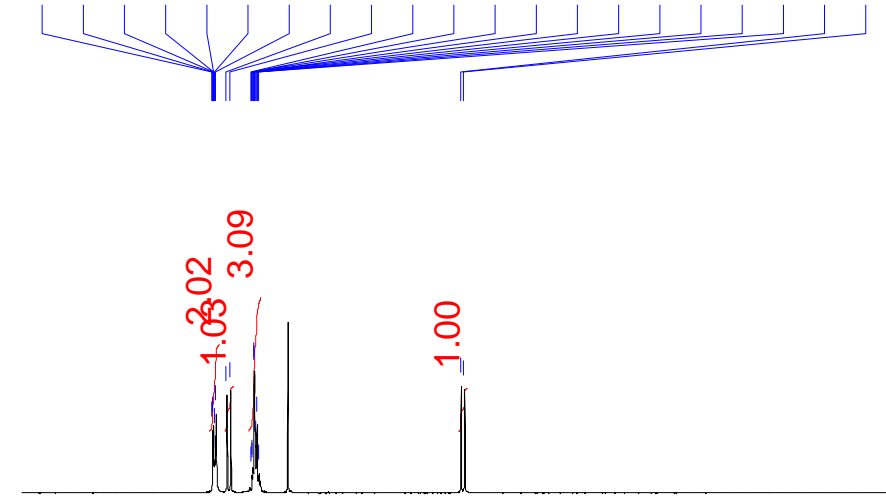

PPM

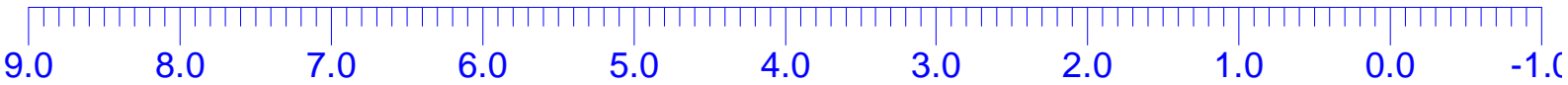

${ }^{13} \mathrm{C}\left\{{ }^{1} \mathrm{H}\right\}$ NMR $\left(100 \mathrm{MHz}, \mathrm{CDCl}_{3}\right)$

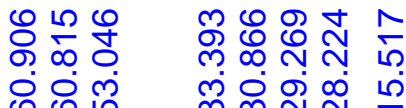

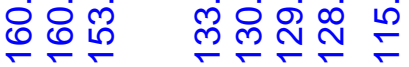

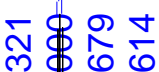

Ní.
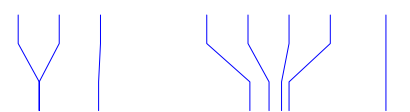

ᄉ

PPM

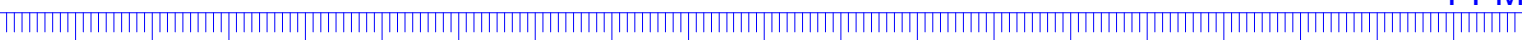

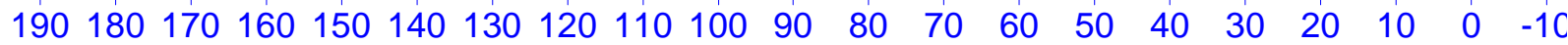


5-iodo-4,6-diphenyl-2H-pyran-2-one (5b)<smiles>O=c1cc(-c2ccccc2)c(I)c(-c2ccccc2)o1</smiles>

${ }^{1} \mathrm{H}$ NMR (400 MHz, $\left.\mathrm{CDCl}_{3}\right)$

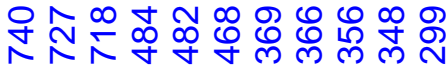

৪

ヘ
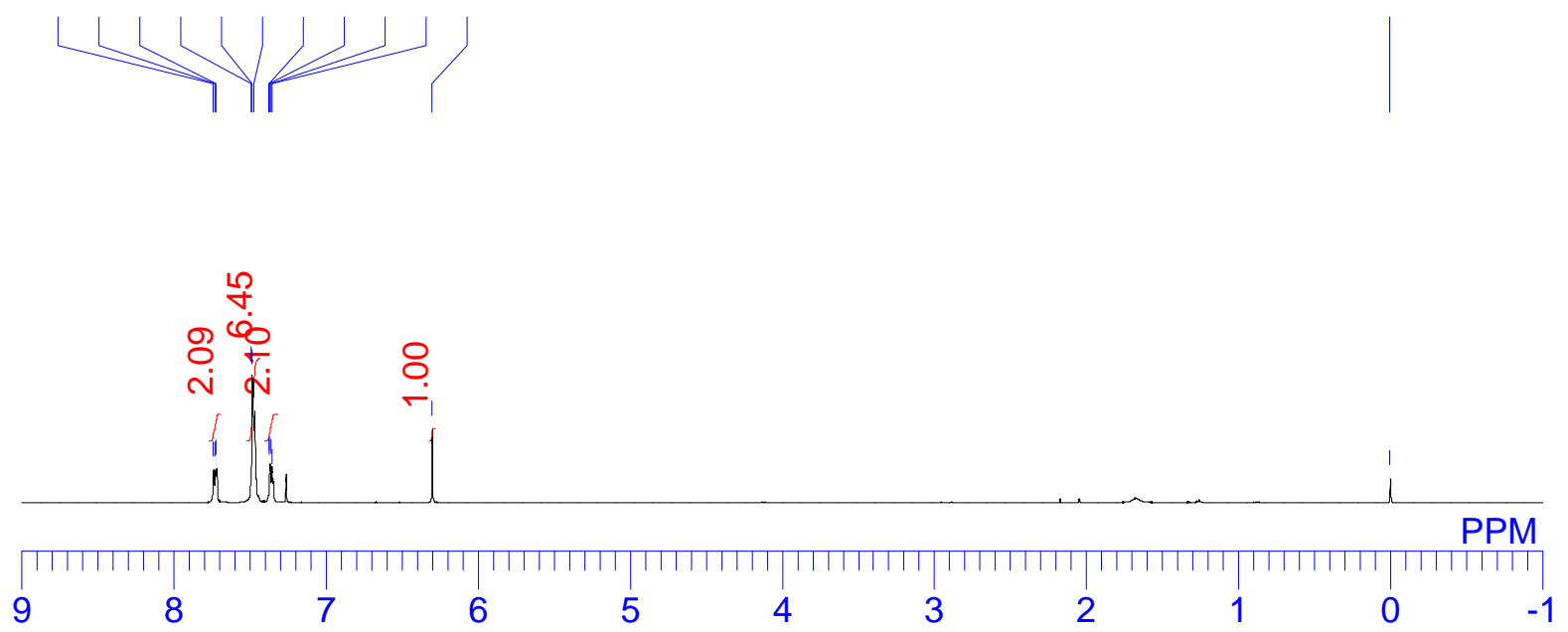

${ }^{13} \mathrm{C}\left\{{ }^{1} \mathrm{H}\right\}$ NMR $\left(100 \mathrm{MHz}, \mathrm{CDCl}_{3}\right)$

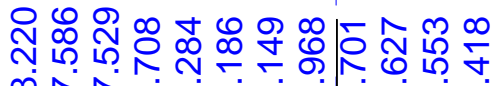

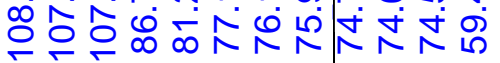

유유ํ유 유

๗ंঙ்

PPM

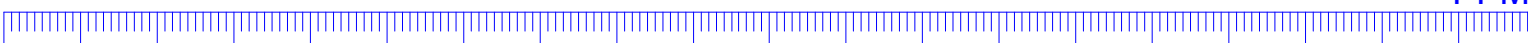

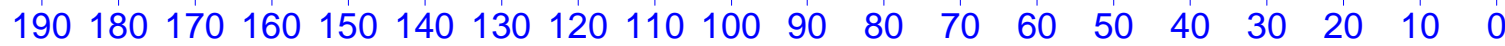


3-ethyl-5-iodo-4,6-diphenyl-2H-pyran-2-one (5c)<smiles>CCc1c(-c2ccccc2)c(I)c(-c2ccccc2)oc1=O</smiles>

${ }^{1} \mathrm{H}$ NMR (400 MHz, $\left.\mathrm{CDCl}_{3}\right)$

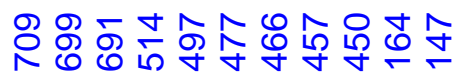

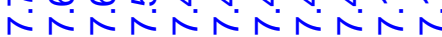

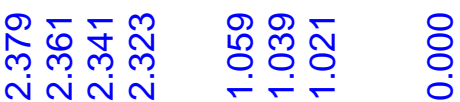
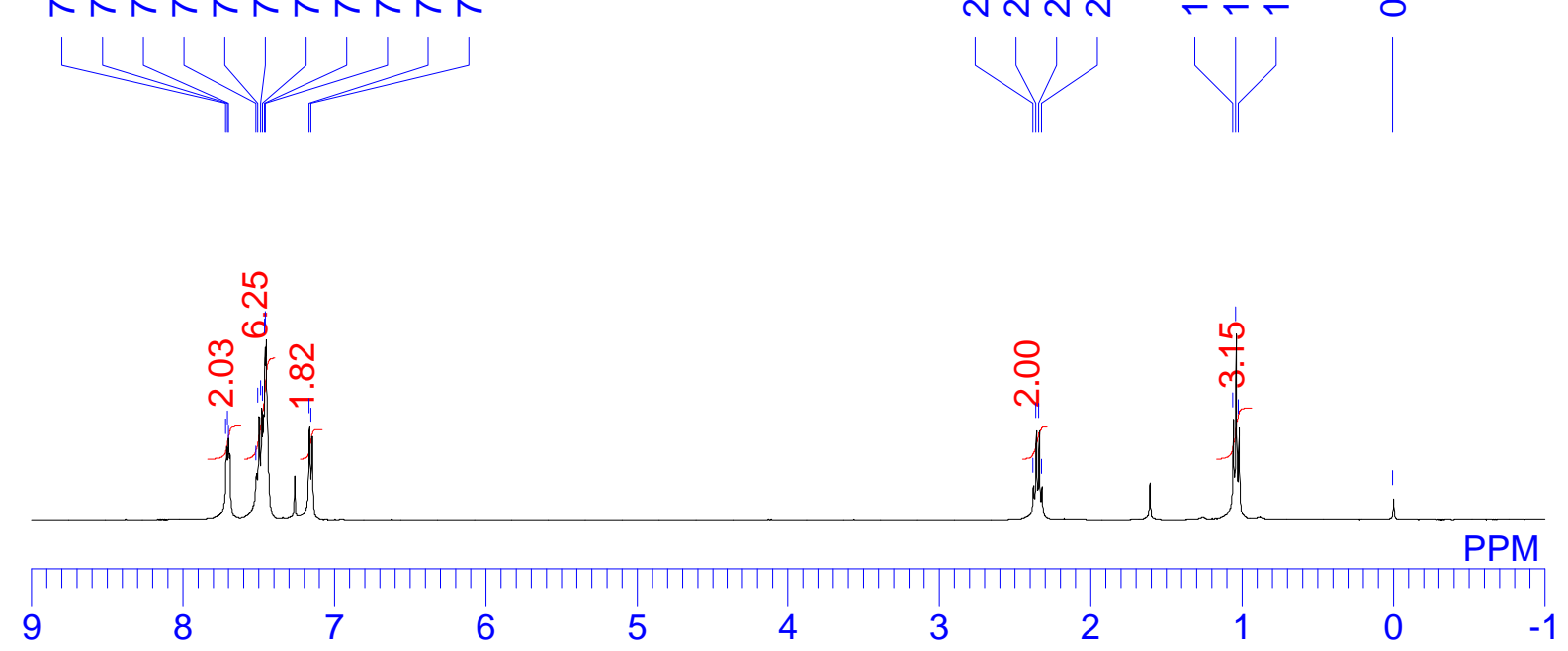

${ }^{13} \mathrm{C}\left\{{ }^{1} \mathrm{H}\right\}$ NMR $\left(100 \mathrm{MHz}, \mathrm{CDCl}_{3}\right)$

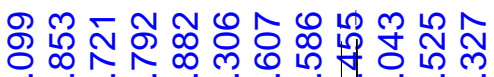

กิن่

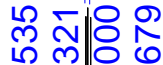

ヘペ余

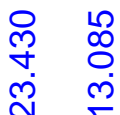
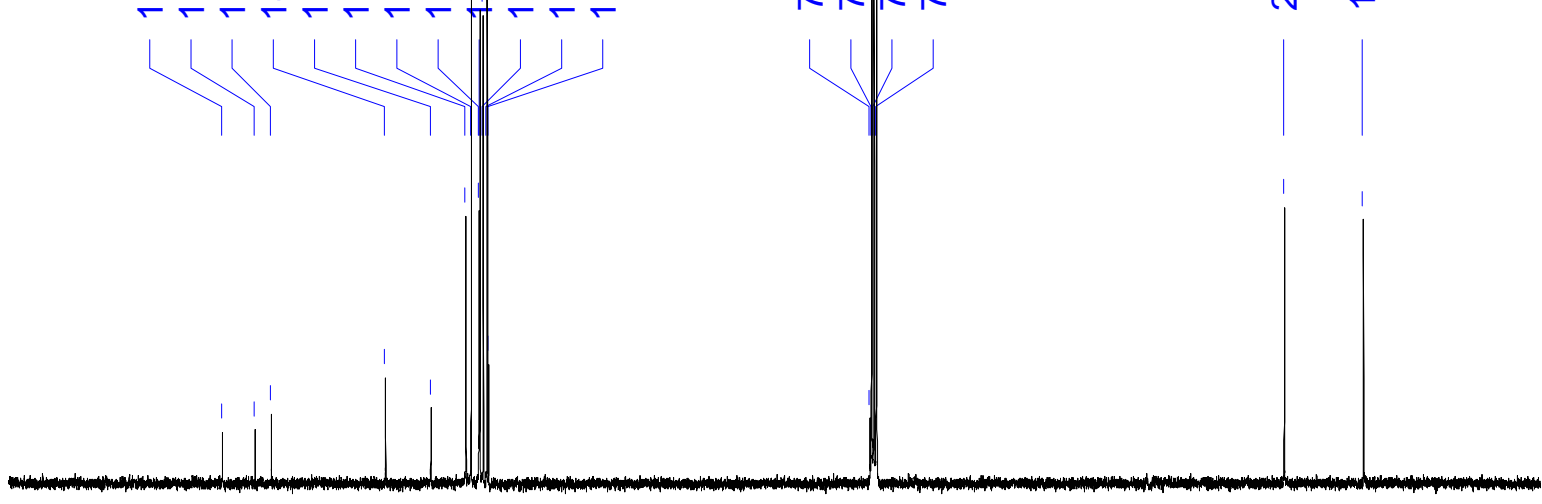

PPM

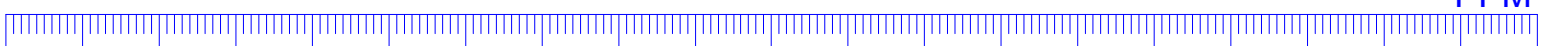

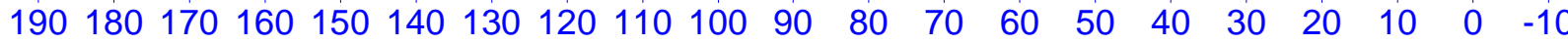


5-iodo-6-(p-tolyl)-2H-pyran-2-one (5d)<smiles>Cc1ccc(-c2oc(=O)ccc2I)cc1</smiles>

${ }^{1} \mathrm{H}$ NMR (400 MHz, $\mathrm{CDCl}_{3}$ )

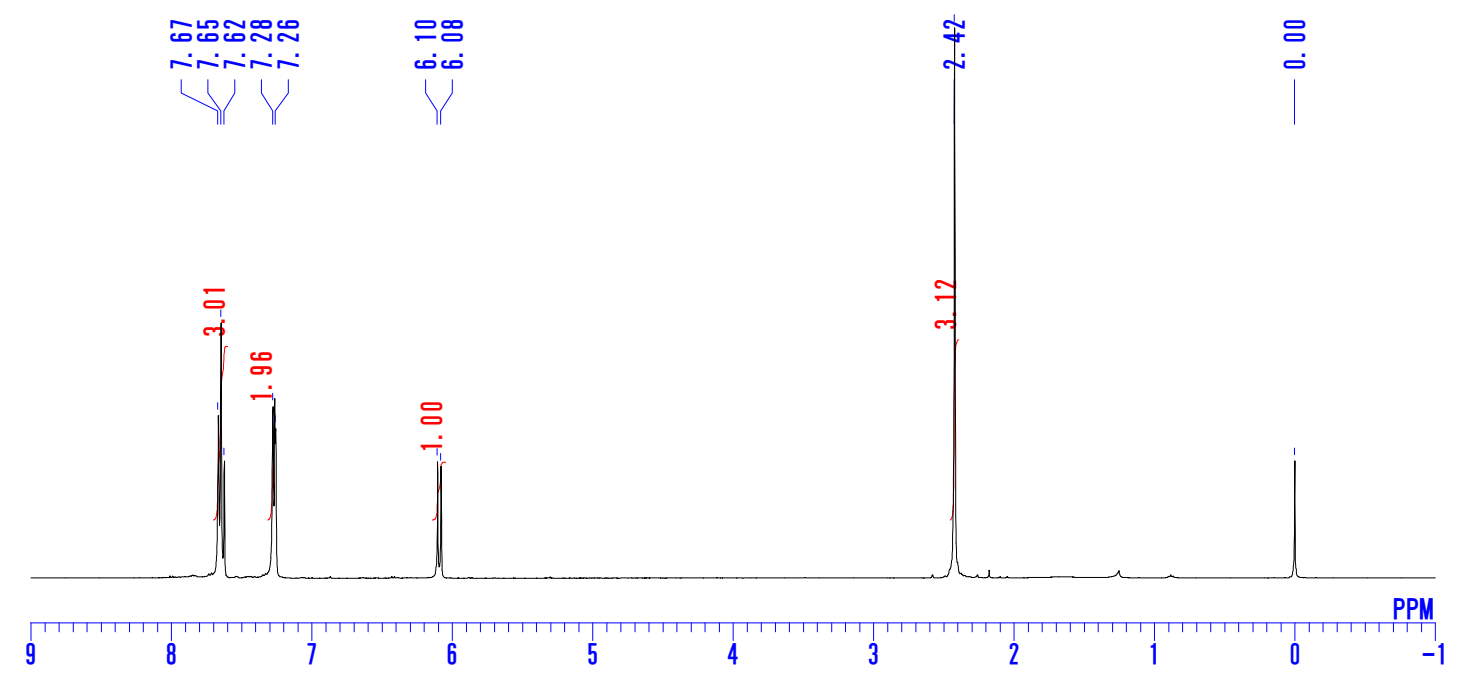

${ }^{13} \mathrm{C}\left\{{ }^{1} \mathrm{H}\right\} \operatorname{NMR}\left(100 \mathrm{MHz}, \mathrm{CDCl}_{3}\right)$

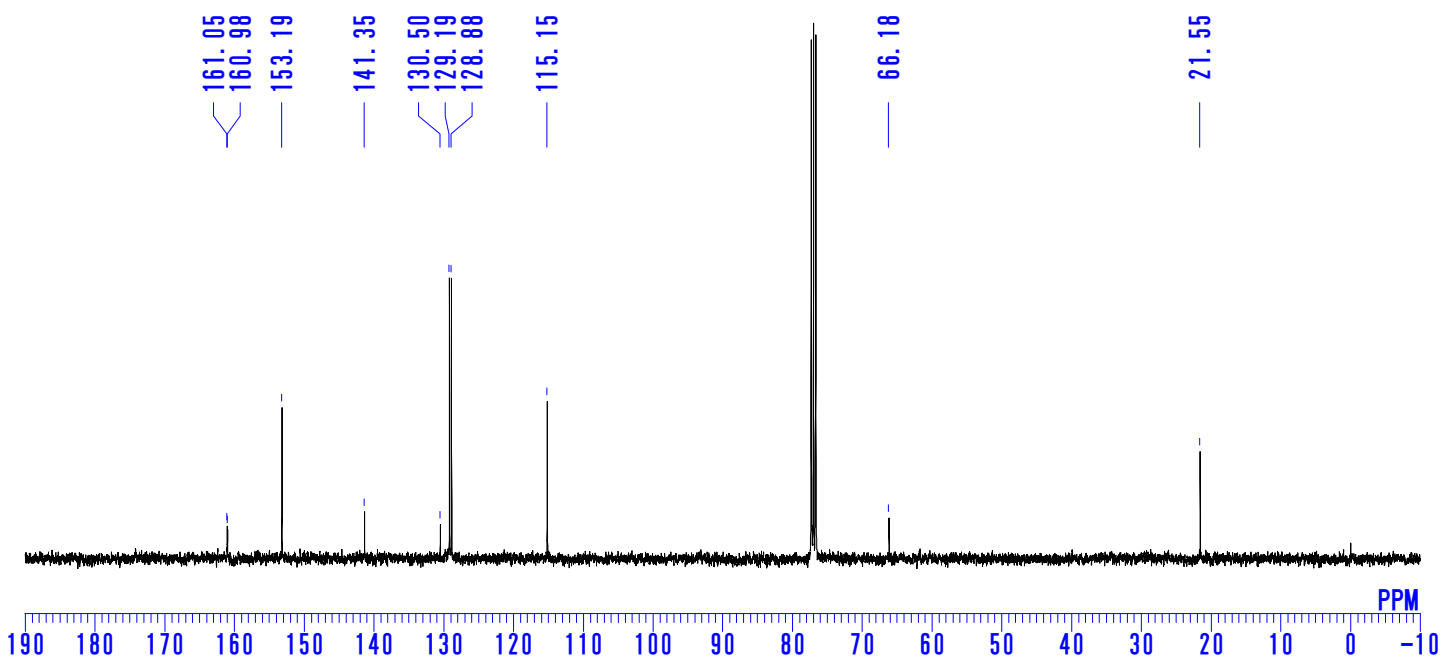


6-\{4-(tert-butyl)phenyl $\}-5$-iodo-2H-pyran-2-one (5e)<smiles>CC(C)(C)c1ccc(-c2oc(=O)ccc2I)cc1</smiles>

${ }^{1} \mathrm{H}$ NMR (400 MHz, $\mathrm{CDCl}_{3}$ )
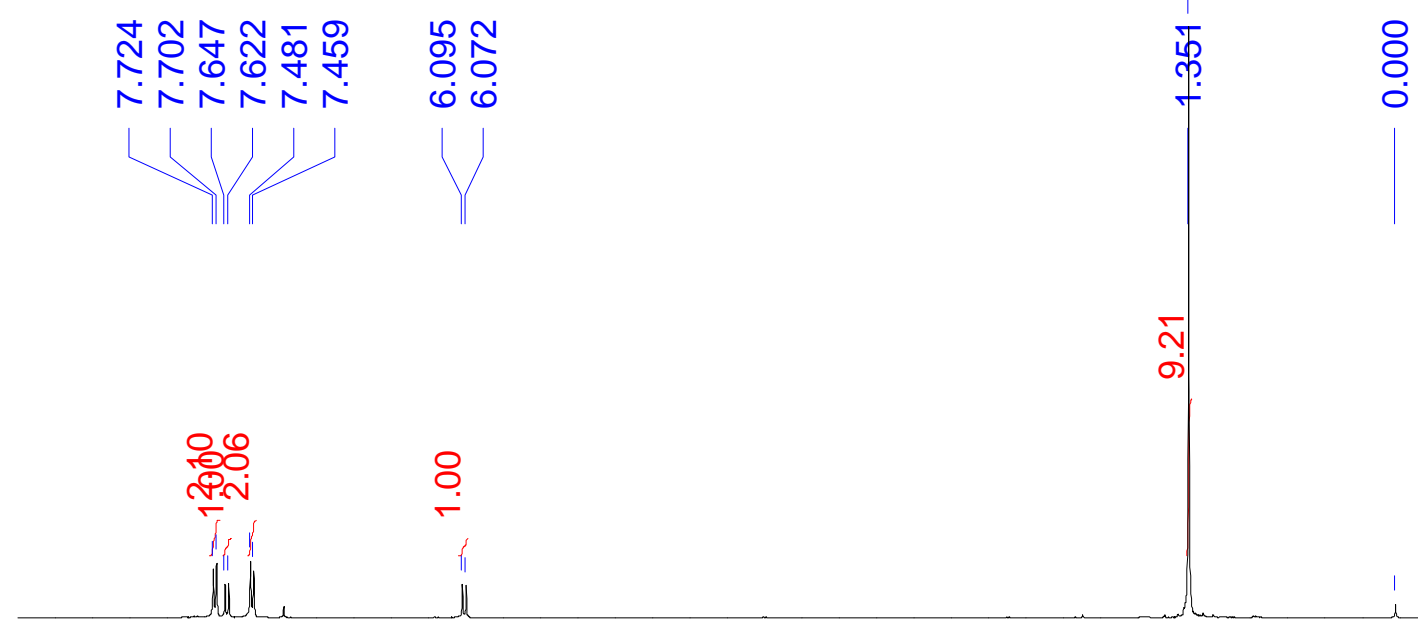

PPM

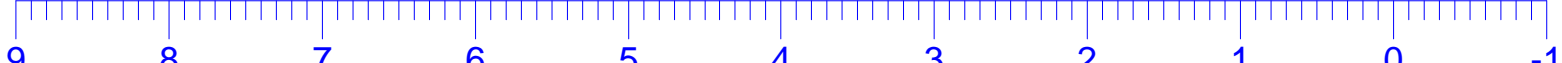

${ }^{13} \mathrm{C}\left\{{ }^{1} \mathrm{H}\right\} \mathrm{NMR}\left(100 \mathrm{MHz}, \mathrm{CDCl}_{3}\right)$

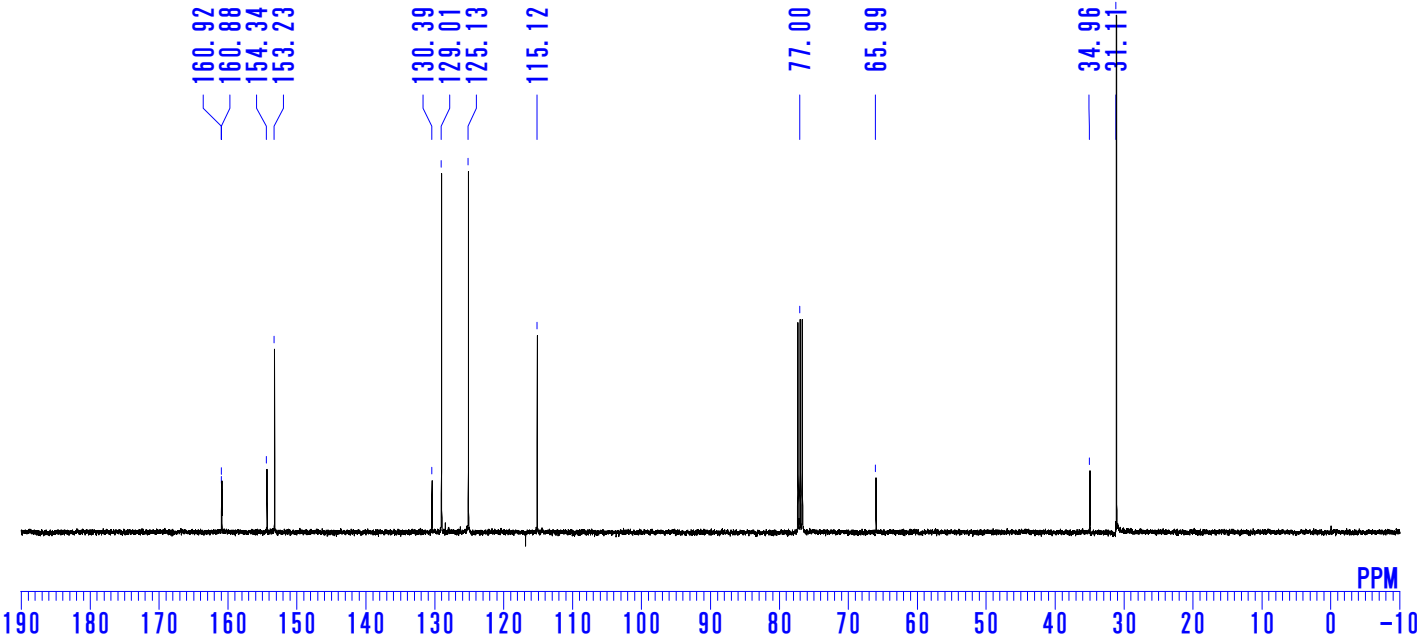


5-iodo-6-(4-methoxyphenyl)-2H-pyran-2-one (5f)<smiles>COc1ccc(-c2oc(=O)ccc2I)cc1</smiles>

${ }^{1} \mathrm{H} \mathrm{NMR}\left(400 \mathrm{MHz}, \mathrm{CDCl}_{3}\right)$
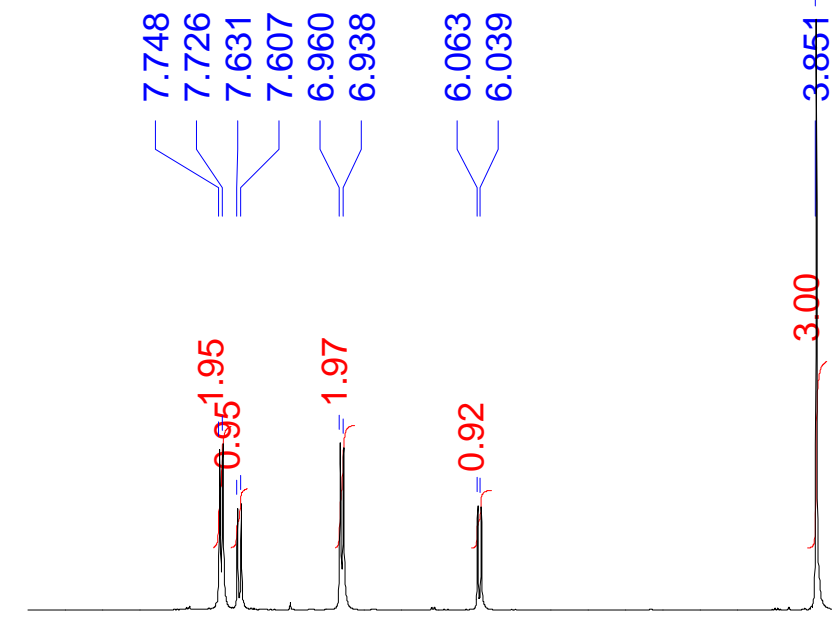

PPM

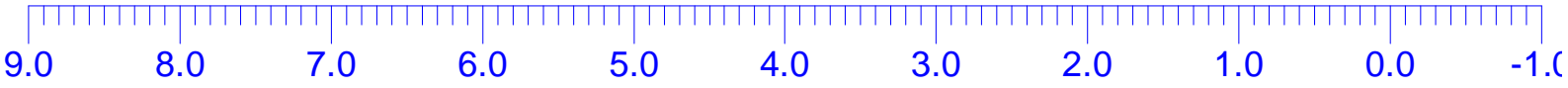

${ }^{13} \mathrm{C}\left\{{ }^{1} \mathrm{H}\right\}$ NMR $\left(100 \mathrm{MHz}, \mathrm{CDCl}_{3}\right)$

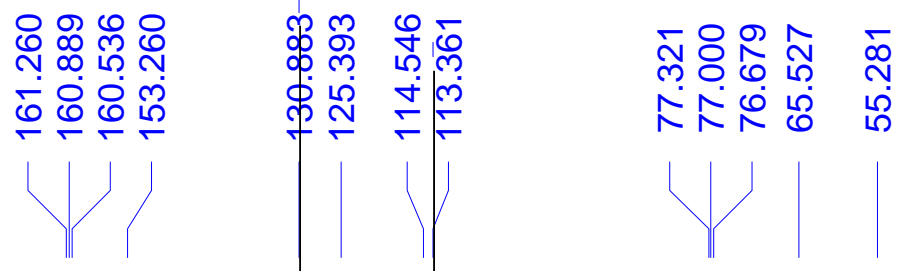


6-(4-chlorophenyl)-5-iodo-2H-pyran-2-one (5g)<smiles>O=c1ccc(I)c(-c2ccc(Cl)cc2)o1</smiles>

${ }^{1} \mathrm{H}$ NMR $\left(400 \mathrm{MHz}, \mathrm{CDCl}_{3}\right)$

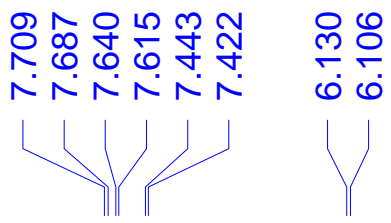

\begin{tabular}{l}
$\circ$ \\
8 \\
\hline
\end{tabular}

กิ กั

Ni

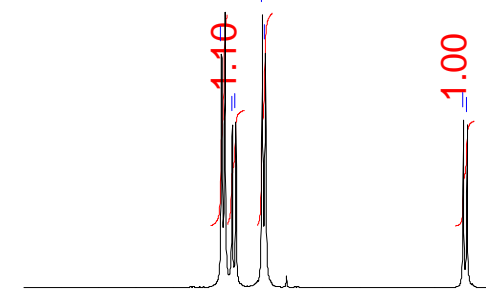

PPM

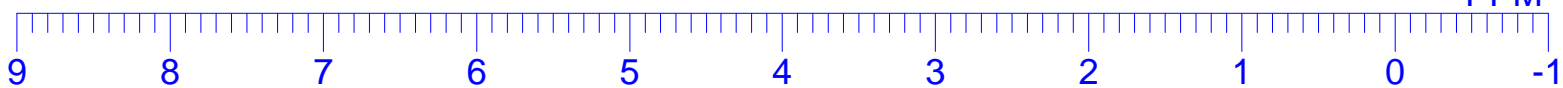

${ }^{13} \mathrm{C}\left\{{ }^{1} \mathrm{H}\right\} \mathrm{NMR}\left(100 \mathrm{MHz}, \mathrm{CDCl}_{3}\right)$

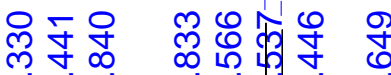

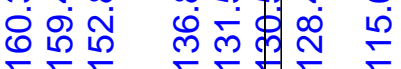

고요

令㲾它
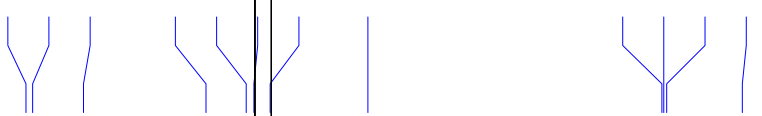

PPM

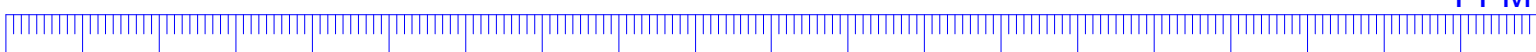

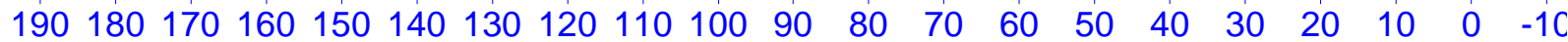


6-(3-fluorophenyl)-5-iodo-2H-pyran-2-one (5h)<smiles>CC(NN)=[R4](N)OC(C)(Cl)Cl</smiles>

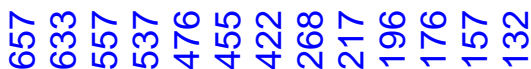

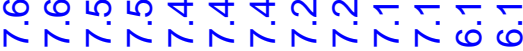
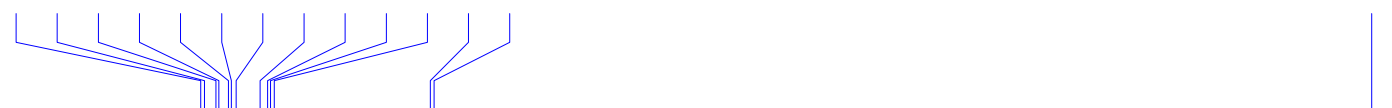

ลे

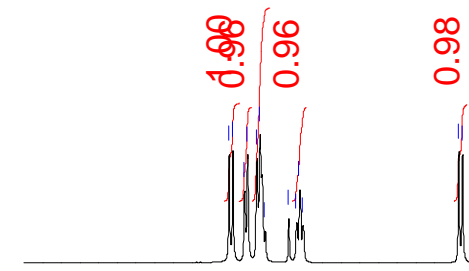

$\stackrel{\infty}{\circ}$

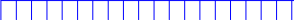

$7 \quad 6$

5

4

43

2

PPM

${ }^{13} \mathrm{C}\left\{{ }^{1} \mathrm{H}\right\} \mathrm{NMR}\left(100 \mathrm{MHz}, \mathrm{CDCl}_{3}\right)$

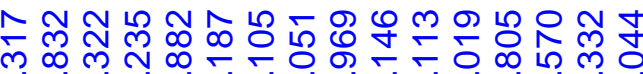

mo 0 \% $N$ in

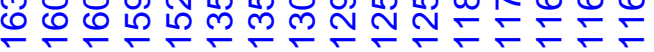

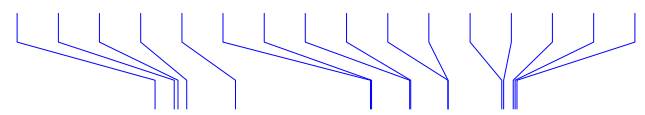

సٓ요용

กิ๊

PPM

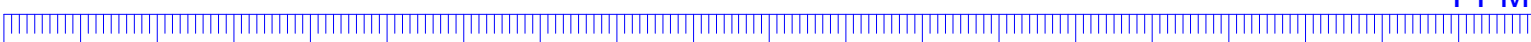

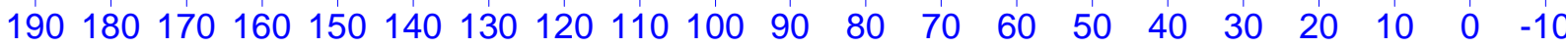


6-hexyl-5-iodo-2H-pyran-2-one (5i)

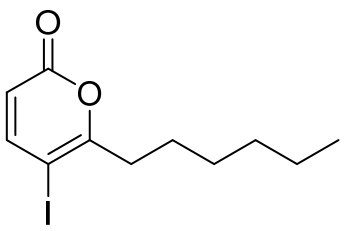

${ }^{1} \mathrm{H} \mathrm{NMR}\left(400 \mathrm{MHz}, \mathrm{CDCl}_{3}\right)$

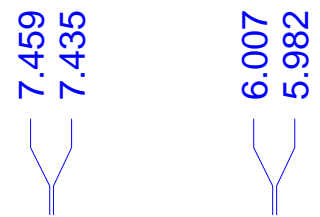

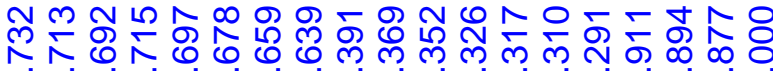

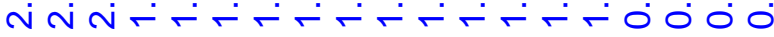

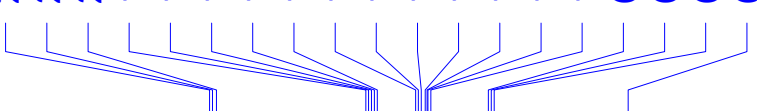

웅.

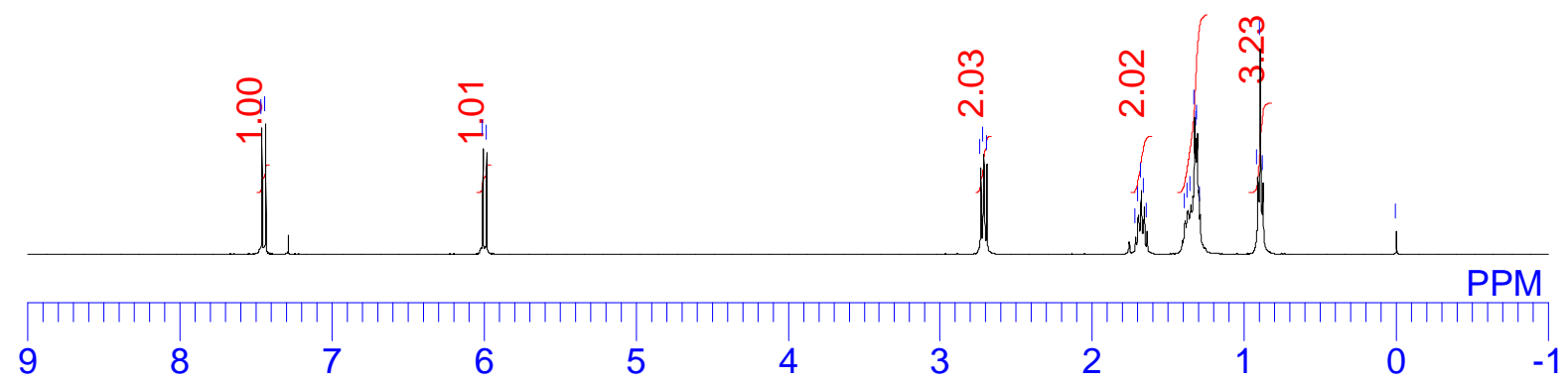

${ }^{13} \mathrm{C}\left\{{ }^{1} \mathrm{H}\right\}$ NMR $\left(100 \mathrm{MHz}, \mathrm{CDCl}_{3}\right)$
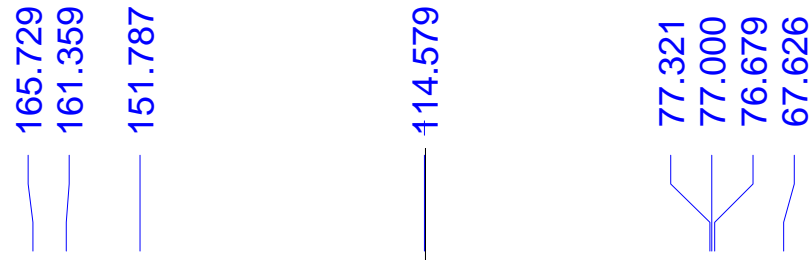

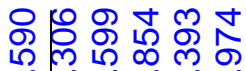
ம் 
6-cyclopropyl-5-iodo-2H-pyran-2-one (5j)

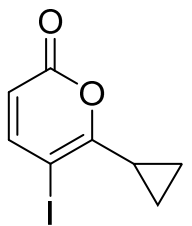

${ }^{1} \mathrm{H}$ NMR $\left(400 \mathrm{MHz}, \mathrm{CDCl}_{3}\right)$

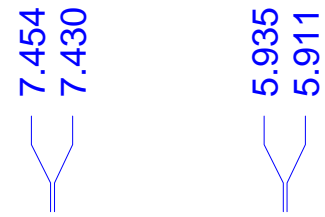

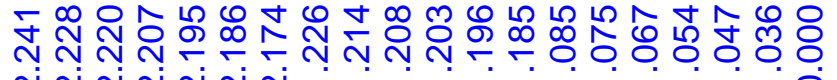

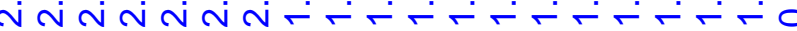
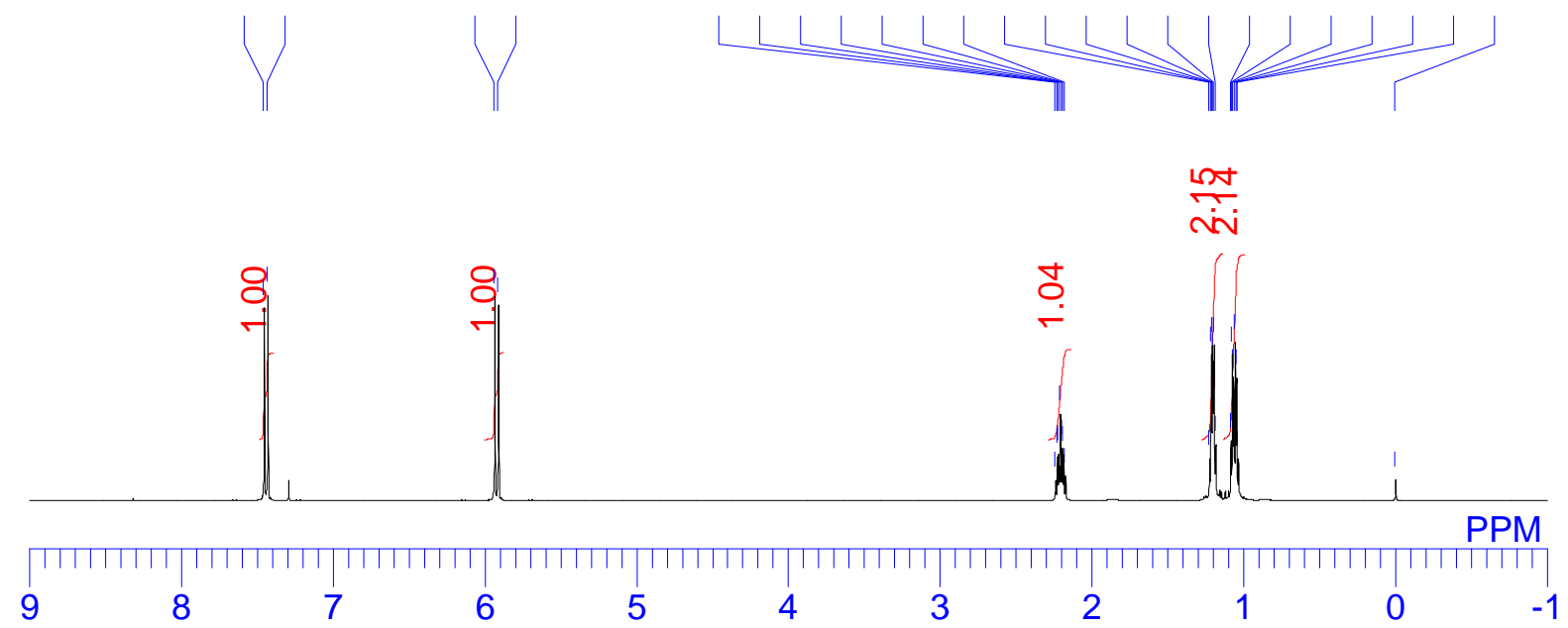

${ }^{13} \mathrm{C}\left\{{ }^{1} \mathrm{H}\right\} \mathrm{NMR}\left(100 \mathrm{MHz}, \mathrm{CDCl}_{3}\right)$
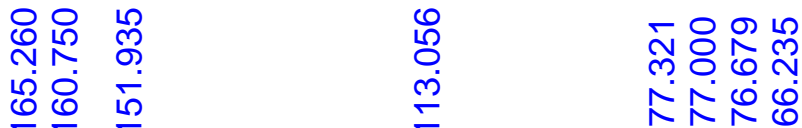

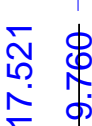

0

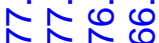
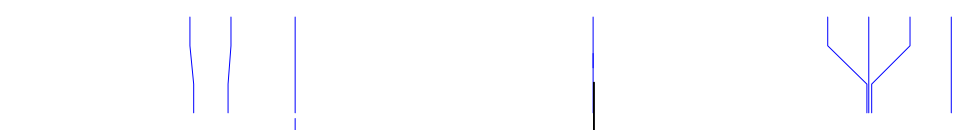

PPM

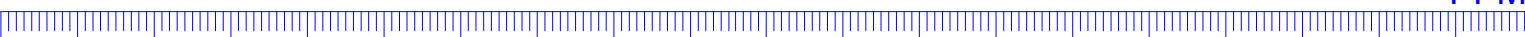

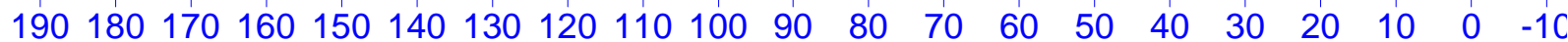


methyl 5-iodo-2-oxo-6-phenyl-2H-pyran-3-carboxylate (5k)<smiles>COC(=O)c1cc(I)c(-c2ccccc2)oc1=O</smiles>

${ }^{1} \mathrm{H}$ NMR $\left(400 \mathrm{MHz}, \mathrm{CDCl}_{3}\right)$

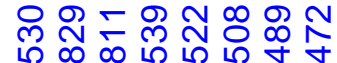

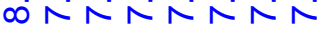

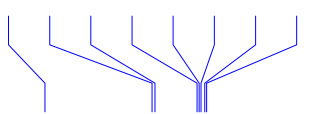

के

৪

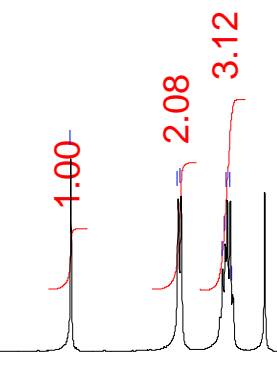

$\stackrel{1}{\circ}$

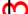

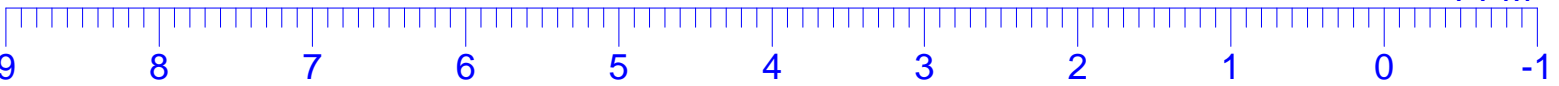

${ }^{13} \mathrm{C}\left\{{ }^{1} \mathrm{H}\right\} \mathrm{NMR}\left(100 \mathrm{MHz}, \mathrm{CDCl}_{3}\right)$

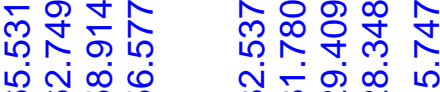

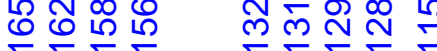
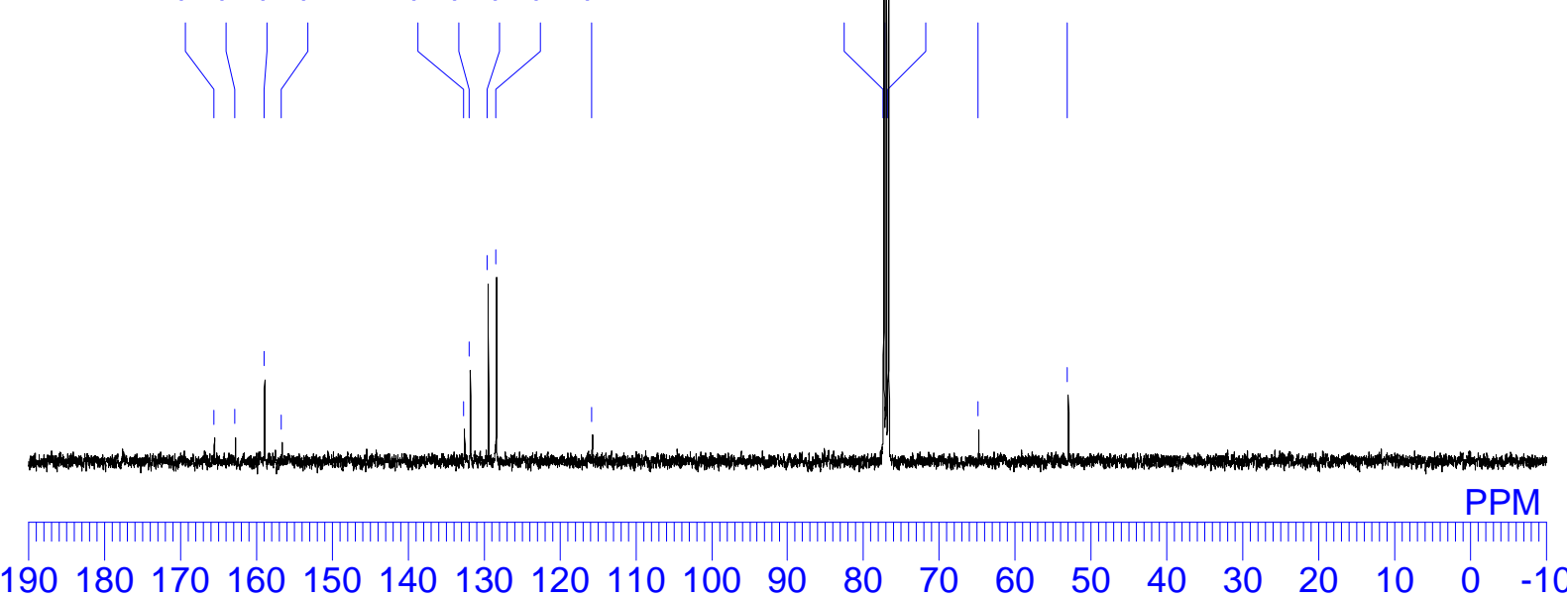

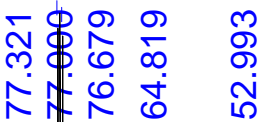

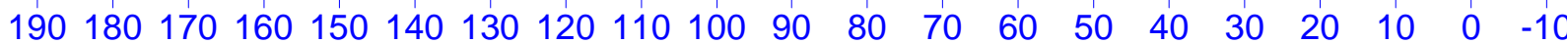


6-cyclopropyl-3-ethyl-5-iodo-4-phenyl-2H-pyran-2-one (5I)<smiles>CCc1c(-c2ccccc2)c(I)c(C2CC2)oc1=O</smiles>

${ }^{1} \mathrm{H}$ NMR $\left(400 \mathrm{MHz}, \mathrm{CDCl}_{3}\right)$

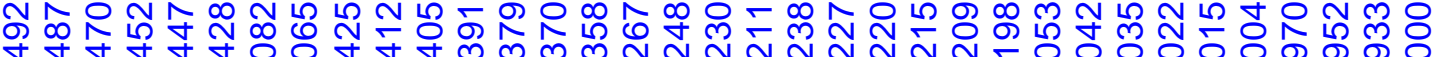

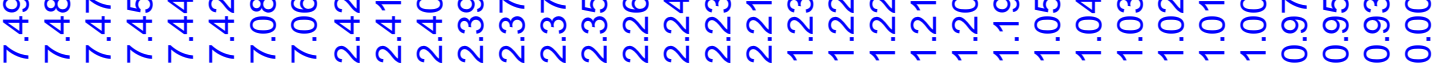

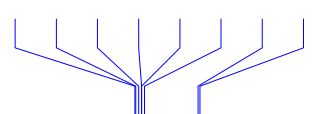

๖े
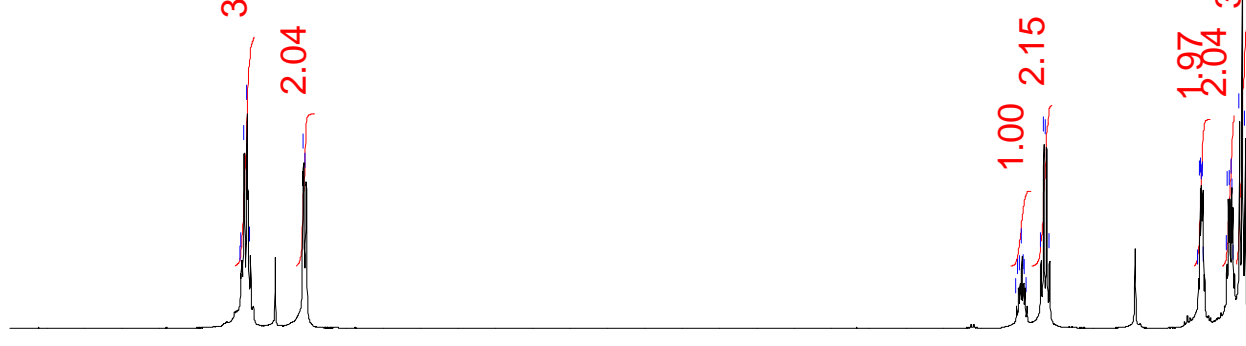

$$
\prod_{9}^{1}
$$

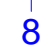

8

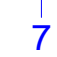

6

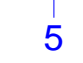

4

3

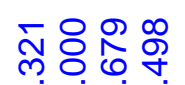
令余定

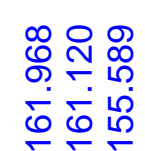

$m$
이

il

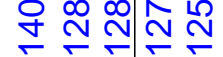
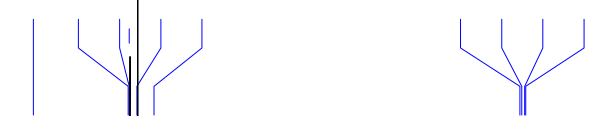

PPM

ता

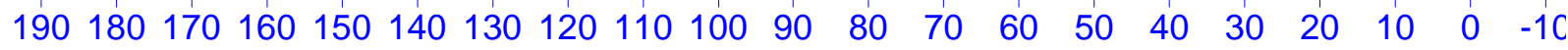


5-iodo-3,4,6-triphenyl-2H-pyran-2-one (5m)<smiles>O=c1oc(-c2ccccc2)c(I)c(-c2ccccc2)c1-c1ccccc1</smiles>

${ }^{1} \mathrm{H} \mathrm{NMR}\left(400 \mathrm{MHz}, \mathrm{CDCl}_{3}\right)$

๓

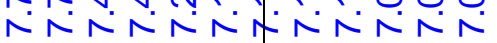

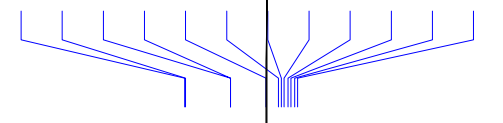

\&

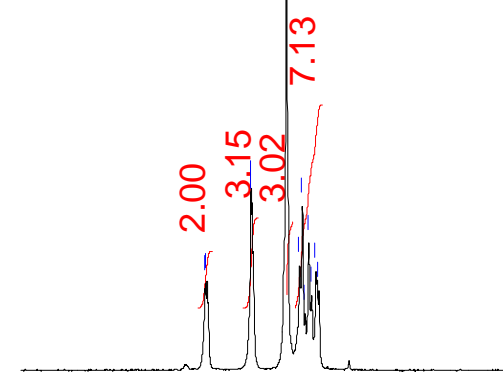

PPM

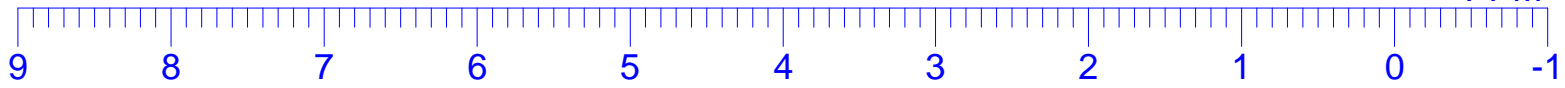

${ }^{13} \mathrm{C}\left\{{ }^{1} \mathrm{H}\right\} \mathrm{NMR}\left(100 \mathrm{MHz}, \mathrm{CDCl}_{3}\right)$

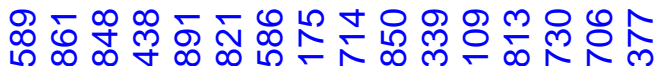

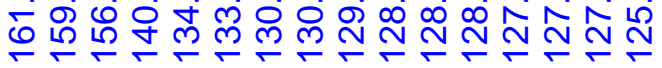
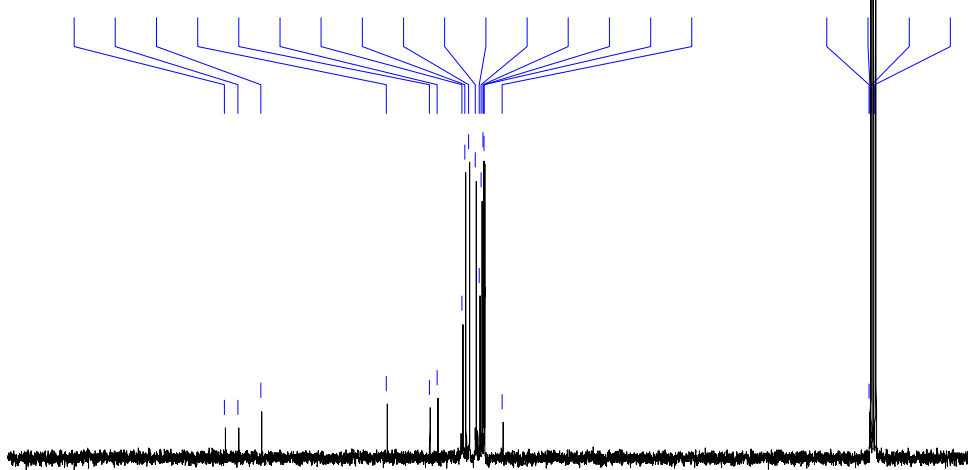

PPM

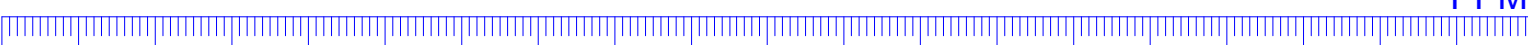

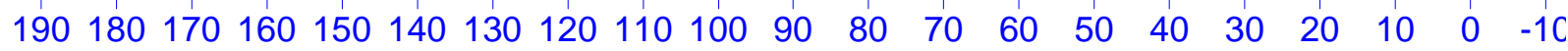


5-bromo-3-ethyl-4,6-diphenyl-2H-pyran-2-one (5n)<smiles>O=c1oc(-c2ccccc2)c(Br)c(-c2ccccc2)c1-c1ccccc1</smiles>

${ }^{1} \mathrm{H}$ NMR $\left(400 \mathrm{MHz}, \mathrm{CDCl}_{3}\right)$

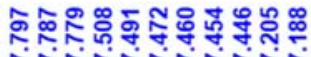
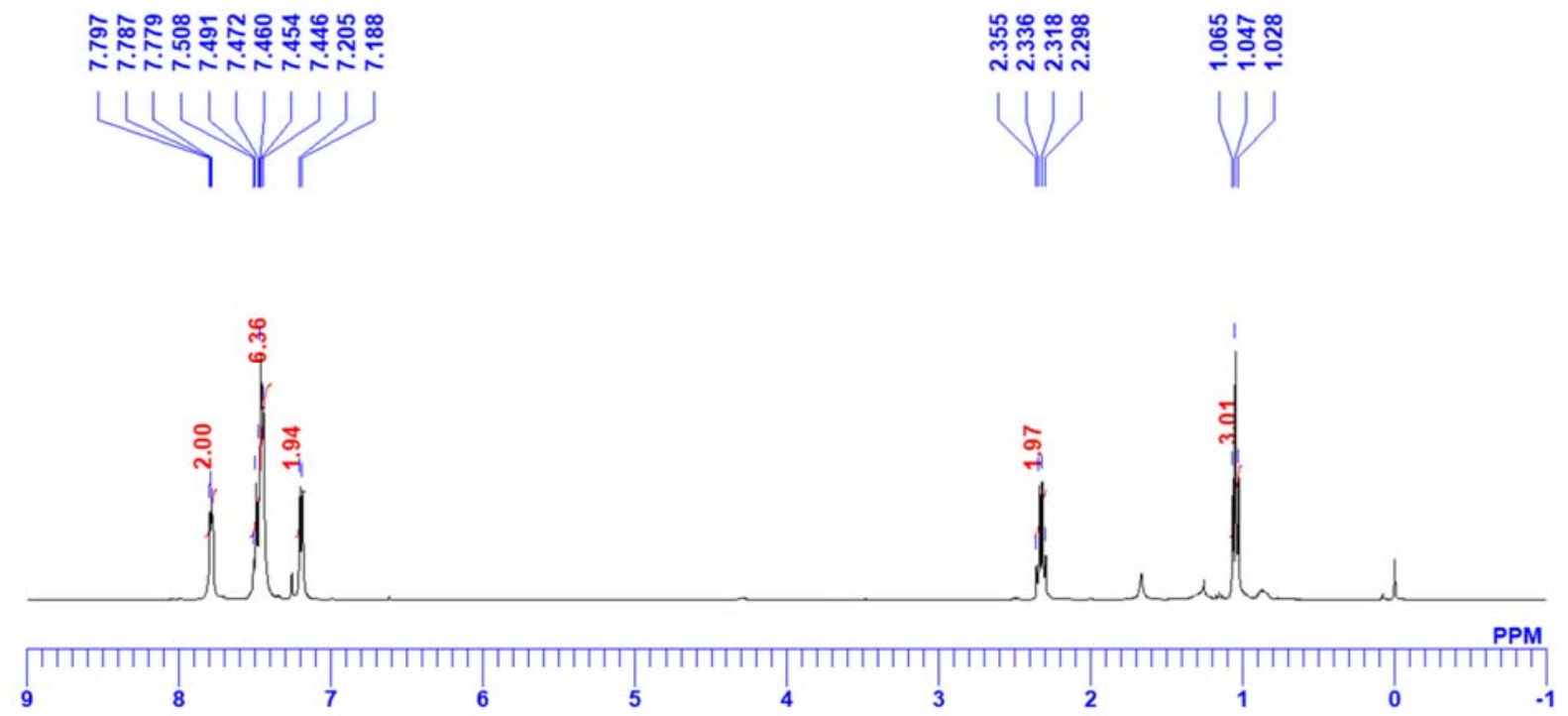

${ }^{13} \mathrm{C}\left\{{ }^{1} \mathrm{H}\right\}$ NMR $\left(100 \mathrm{MHz}, \mathrm{CDCl}_{3}\right)$
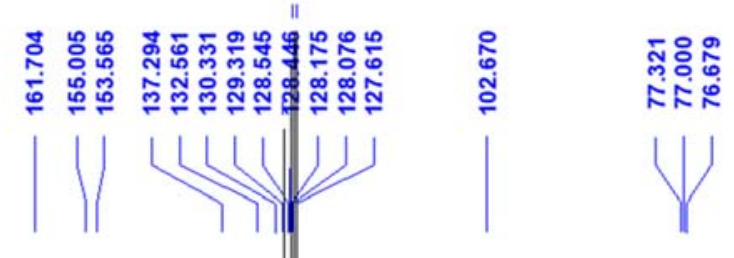

ヘొ

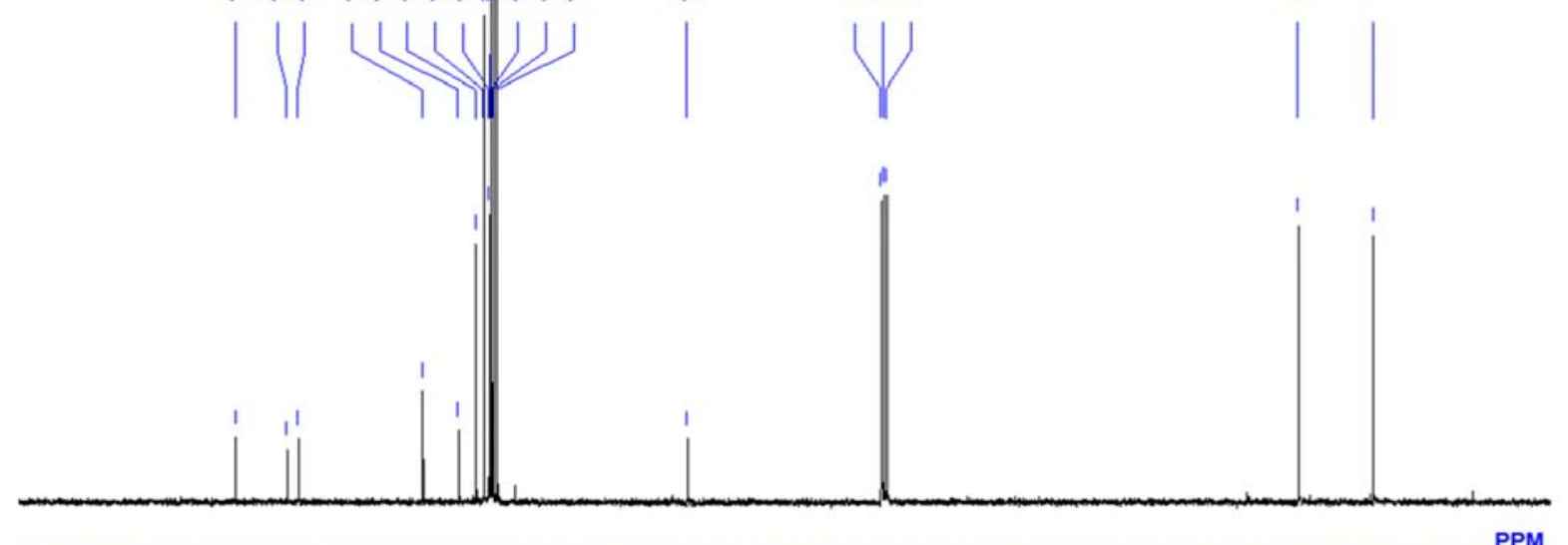

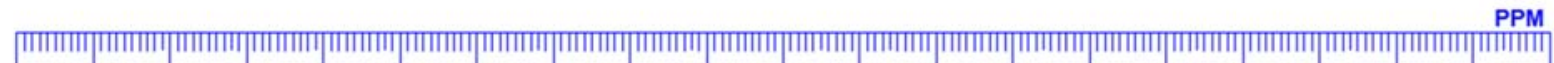

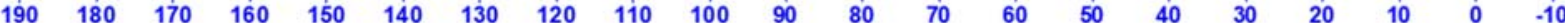


5,6-diphenyl-2H-pyran-2-one (6a)<smiles>O=c1ccc(-c2ccccc2)c(-c2ccccc2)o1</smiles>

${ }^{1} \mathrm{H}$ NMR (400 MHz, $\mathrm{CDCl}_{3}$ )

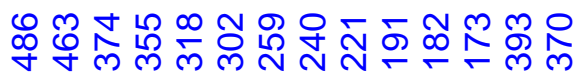

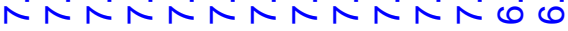

\&
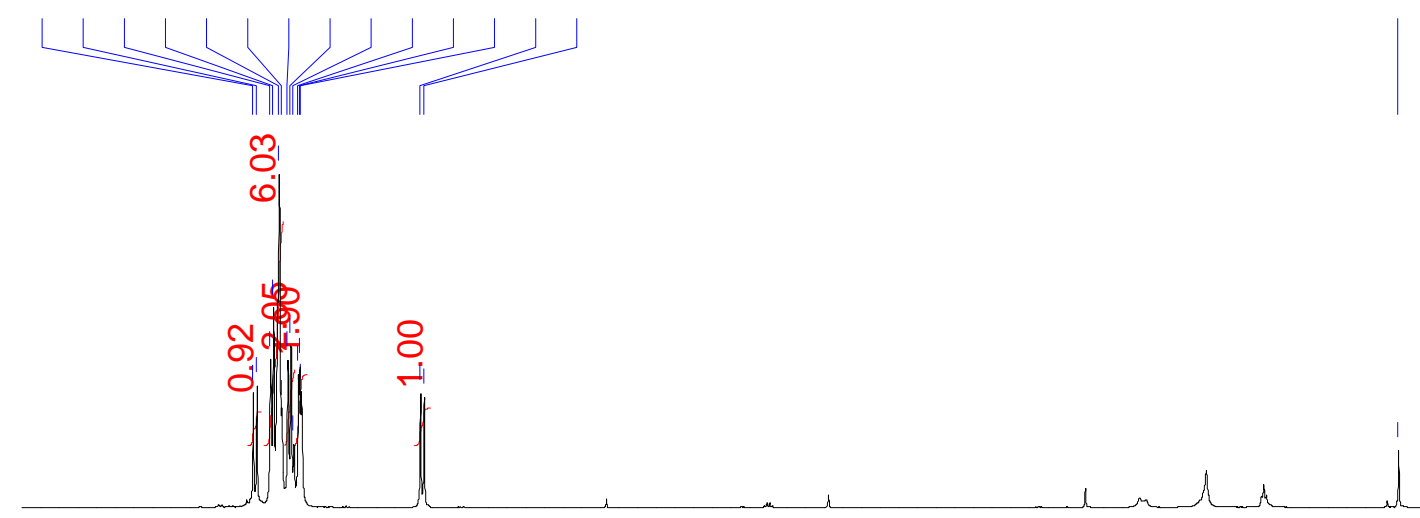

PPM

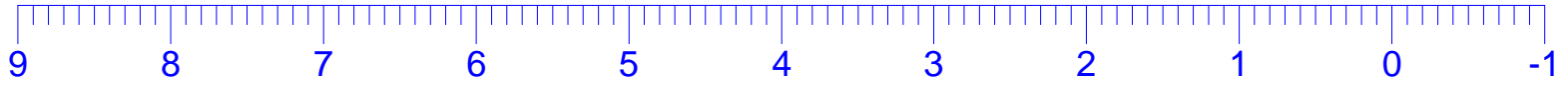

${ }^{13} \mathrm{C}\left\{{ }^{1} \mathrm{H}\right\}$ NMR $\left(100 \mathrm{MHz}, \mathrm{CDCl}_{3}\right)$

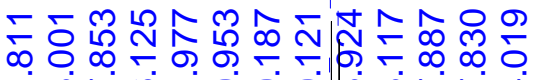
-i

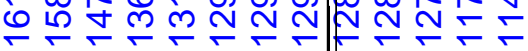

그용

ヘペ余

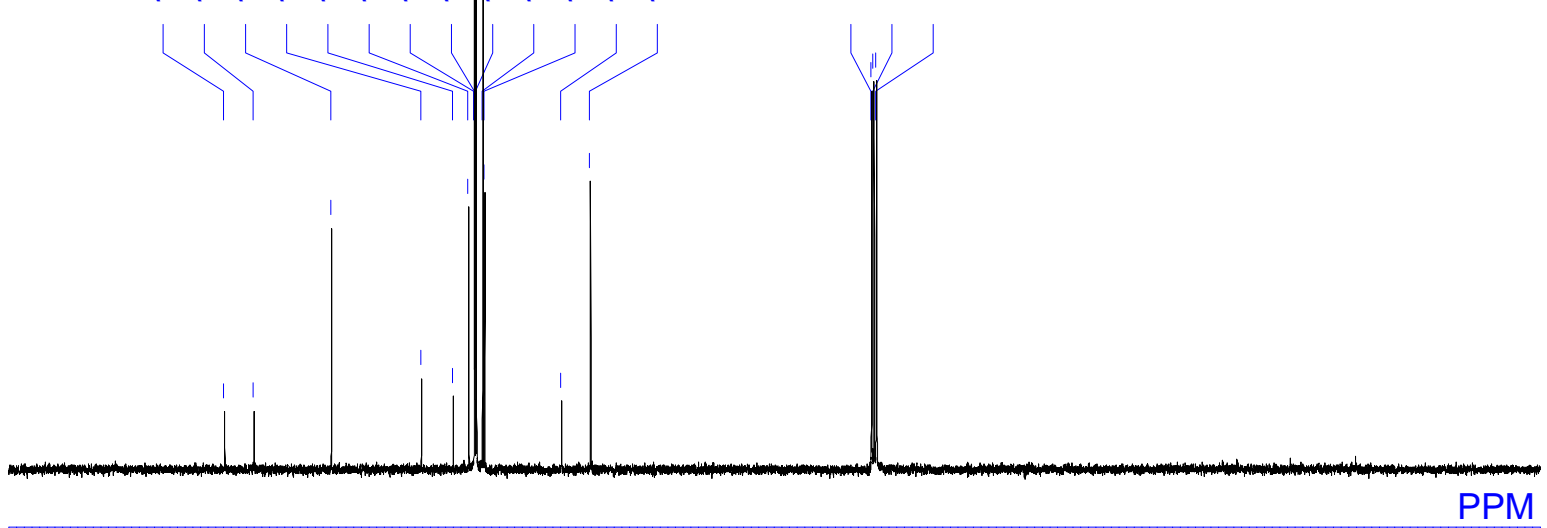

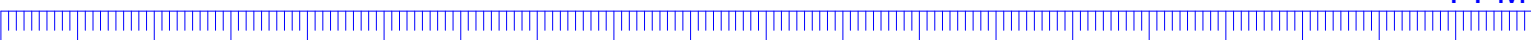

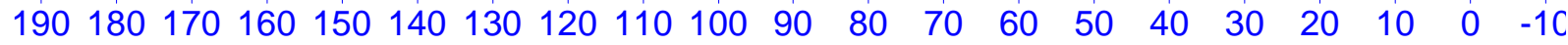


4,5,6-triphenyl-2H-pyran-2-one (6b)<smiles>O=c1cc(-c2ccccc2)c(-c2ccccc2)c(-c2ccccc2)o1</smiles>

${ }^{1} \mathrm{H}$ NMR $\left(400 \mathrm{MHz}, \mathrm{CDCl}_{3}\right)$

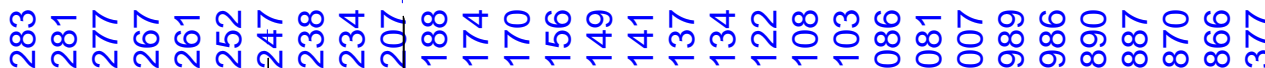

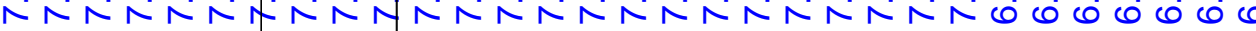

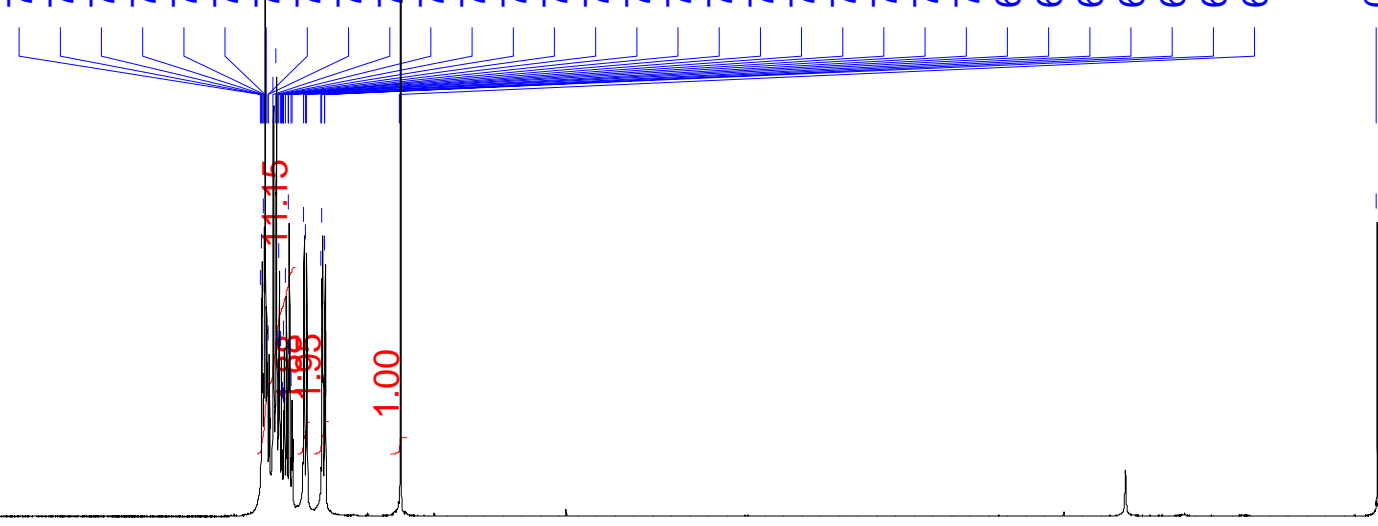

PPM

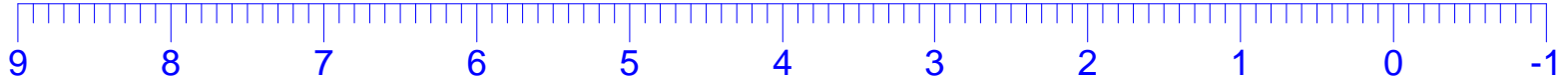

${ }^{13} \mathrm{C}\left\{{ }^{1} \mathrm{H}\right\}$ NMR $\left(100 \mathrm{MHz}, \mathrm{CDCl}_{3}\right)$

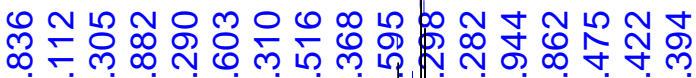

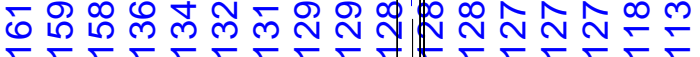

교요

ベペ
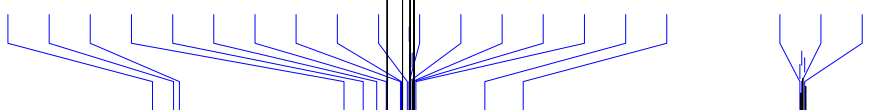

PPM

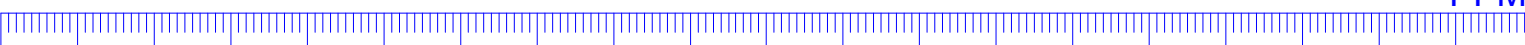

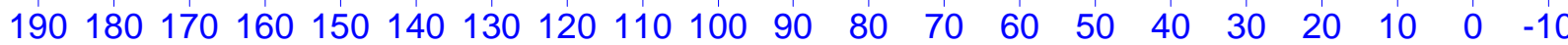


3-ethyl-4,5,6-triphenyl-2H-pyran-2-one (6c)<smiles>CCc1c(-c2ccccc2)c(-c2ccccc2)c(-c2ccccc2)oc1=O</smiles>

${ }^{1} \mathrm{H}$ NMR $\left(400 \mathrm{MHz}, \mathrm{CDCl}_{3}\right)$

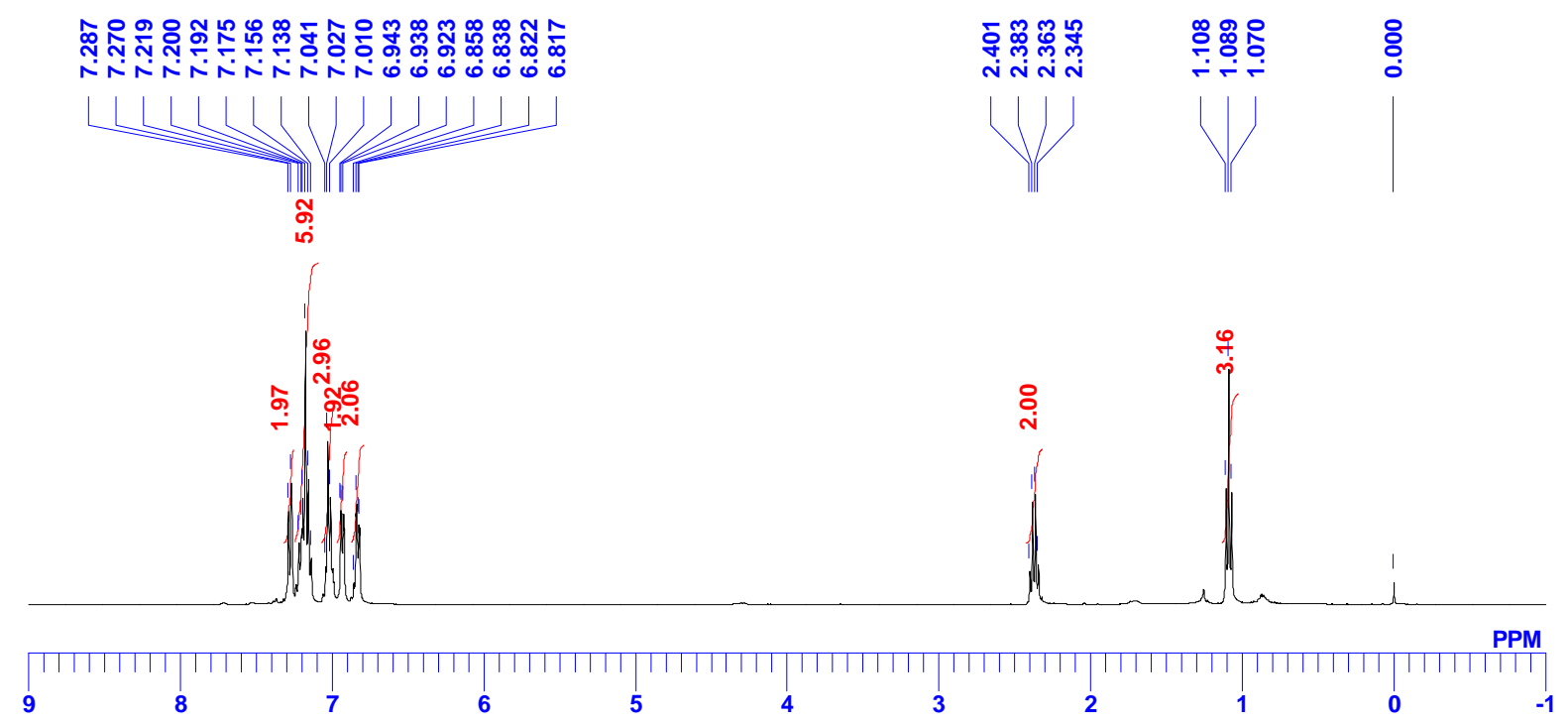

${ }^{13} \mathrm{C}\left\{{ }^{1} \mathrm{H}\right\}$ NMR $\left(100 \mathrm{MHz}, \mathrm{CDCl}_{3}\right)$

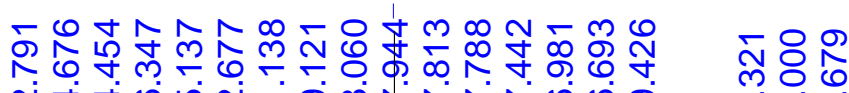

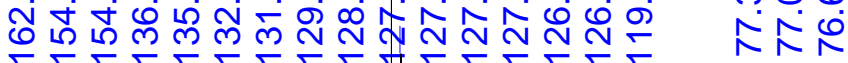

กิ $\stackrel{\infty}{\sim}$

ก $\stackrel{m}{\rightarrow}$
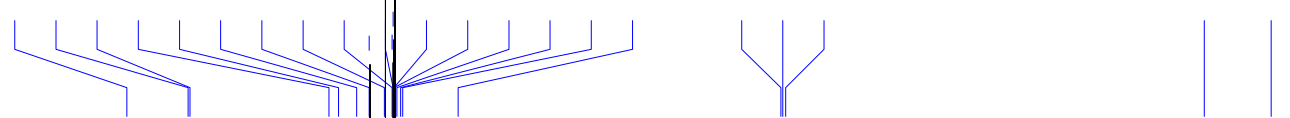

PPM

|

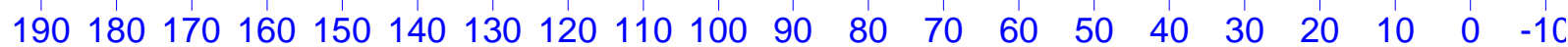


3,4,5,6-tetraphenyl-2H-pyran-2-one (6m)<smiles>O=c1oc(-c2ccccc2)c(-c2ccccc2)c(-c2ccccc2)c1-c1ccccc1</smiles>

${ }^{1} \mathrm{H} \mathrm{NMR}\left(400 \mathrm{MHz}, \mathrm{CDCl}_{3}\right)$

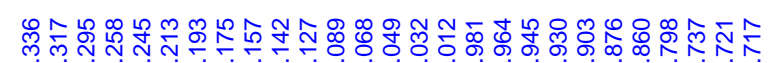

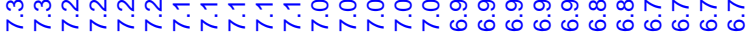

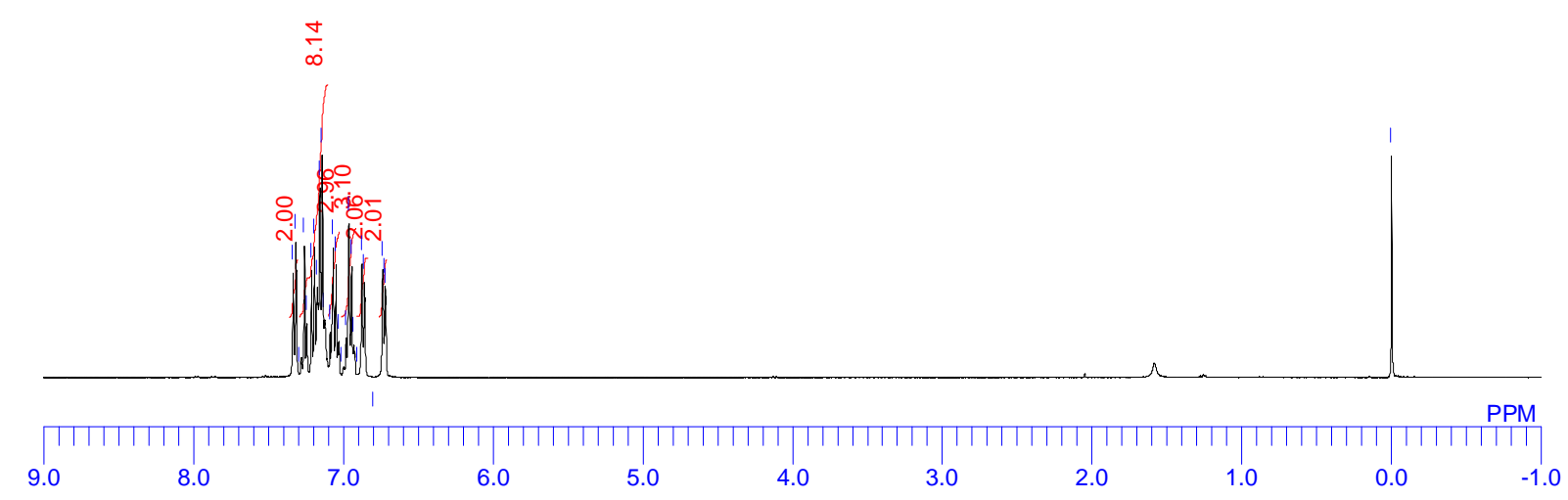

${ }^{13} \mathrm{C}\left\{{ }^{1} \mathrm{H}\right\}$ NMR $\left(100 \mathrm{MHz}, \mathrm{CDCl}_{3}\right)$

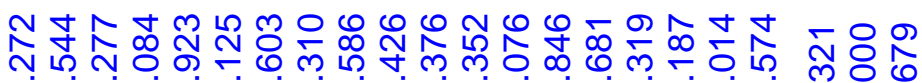

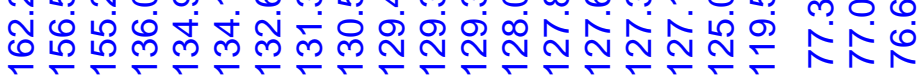
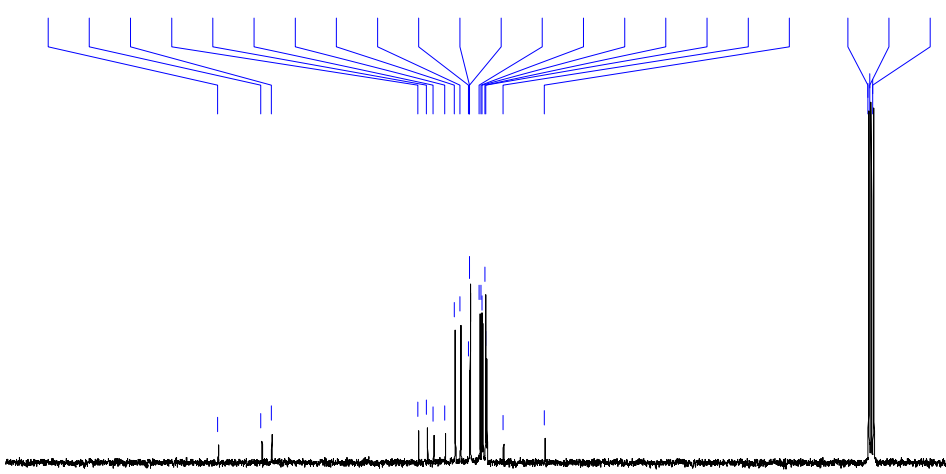

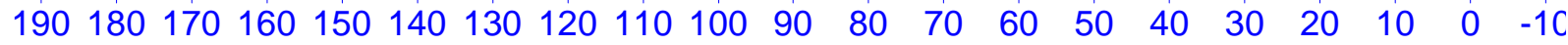


6-cyclopropyl-3-ethyl-4,5-diphenyl-2H-pyran-2-one (6l)<smiles>CCc1c(-c2ccccc2)c(-c2ccccc2)c(C2CC2)oc1=O</smiles>

${ }^{1} \mathrm{H} \mathrm{NMR}\left(400 \mathrm{MHz}, \mathrm{CDCl}_{3}\right)$

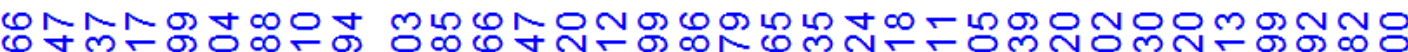

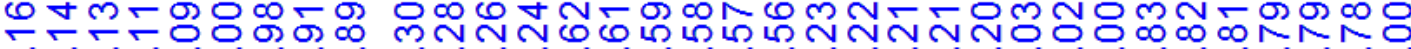

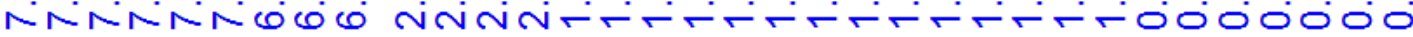
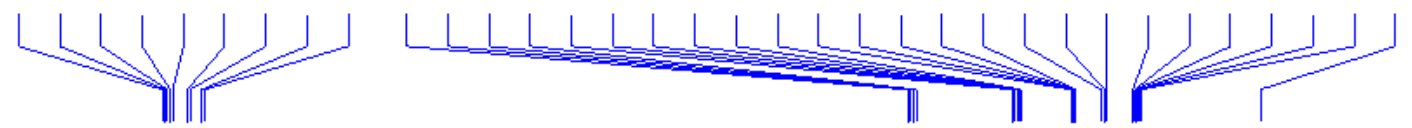

స

$\omega$

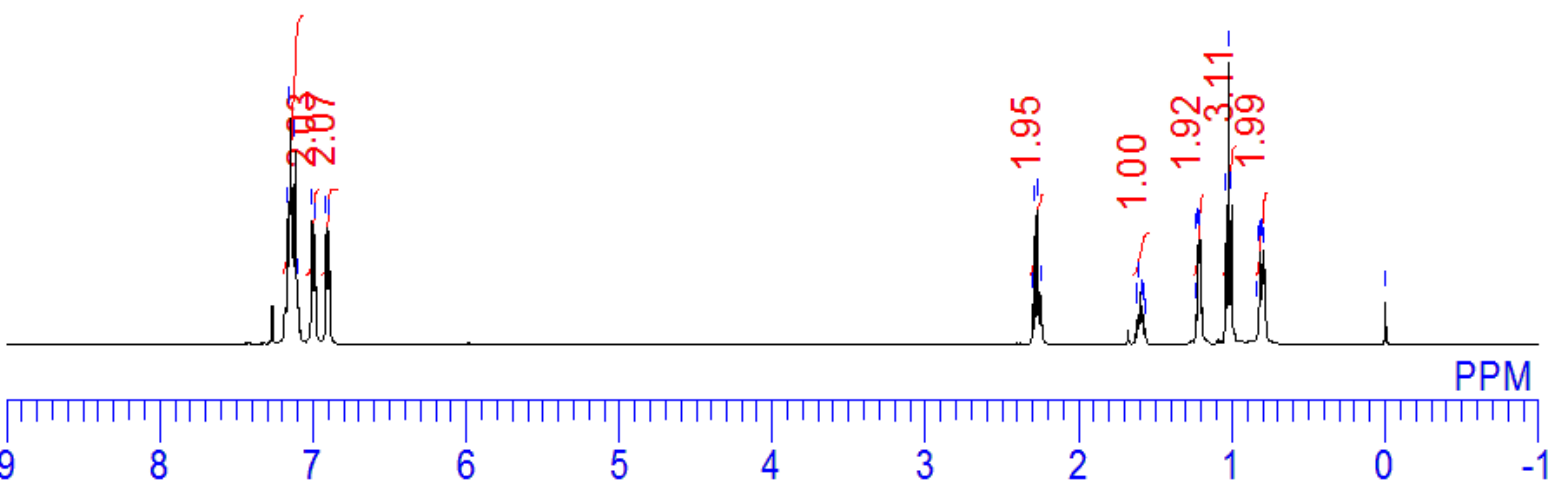

${ }^{13} \mathrm{C}\left\{{ }^{1} \mathrm{H}\right\} \mathrm{NMR}\left(100 \mathrm{MHz}, \mathrm{CDCl}_{3}\right)$

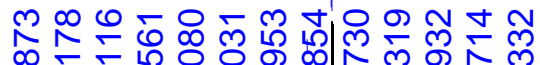

m

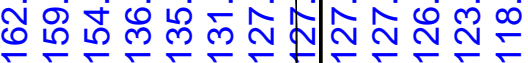

긍요

กิ์

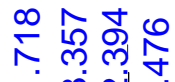
ล่ $\stackrel{\rightarrow}{\rightarrow} \underset{\sim}{\infty}$
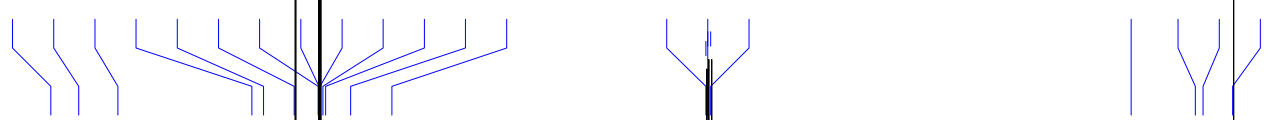

PPM

|

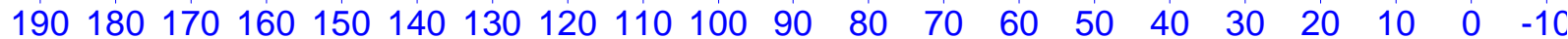


6-cyclopropyl-3-ethyl-5-(4-methoxyphenyl)-4-phenyl-2H-pyran-2-one (6n)<smiles>CCc1c(-c2ccccc2)c(-c2ccc(OC)cc2)oc(=O)c1C1CC1</smiles>

${ }^{1} \mathrm{H} \mathrm{NMR}\left(400 \mathrm{MHz}, \mathrm{CDCl}_{3}\right)$

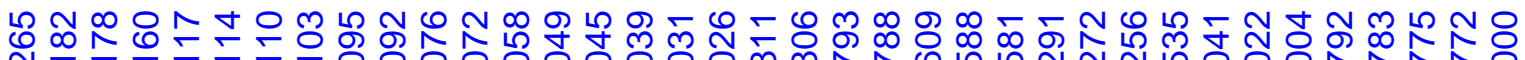

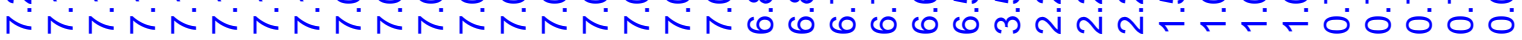
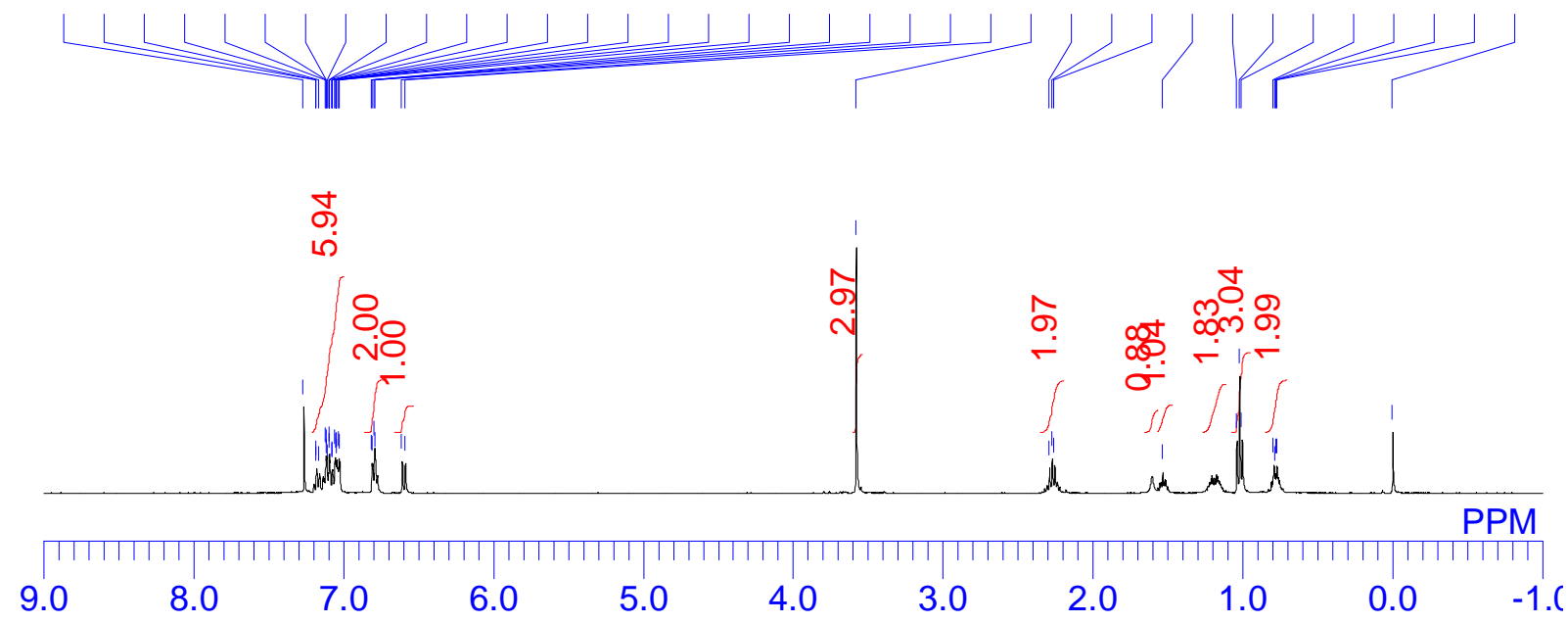

${ }^{13} \mathrm{C}\left\{{ }^{1} \mathrm{H}\right\}$ NMR $\left(100 \mathrm{MHz}, \mathrm{CDCl}_{3}\right)$

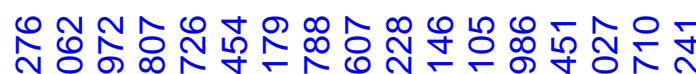

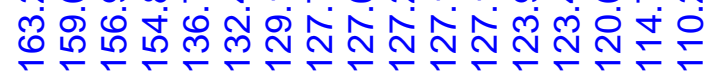

స్요요

令昘

$\stackrel{\text { ֻn }}{\circ}$

オ ㄱำ
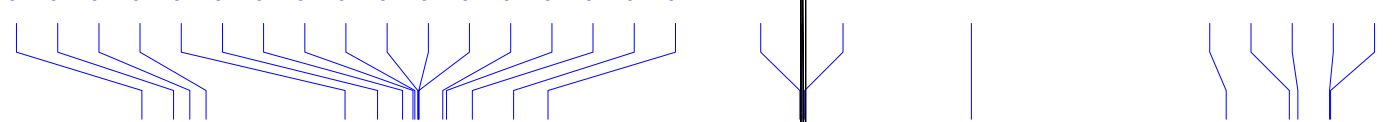

PPM

ता

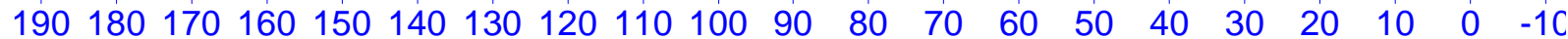


6-cyclopropyl-3-ethyl-5-(4-nitrophenyl)-4-phenyl-2H-pyran-2-one (6o)<smiles>CCc1c(-c2ccccc2)c(-c2ccc([N+](=O)[O-])cc2)c(C2CC2)oc1=O</smiles>

${ }^{1} \mathrm{H} \mathrm{NMR}\left(400 \mathrm{MHz}, \mathrm{CDCl}_{3}\right)$

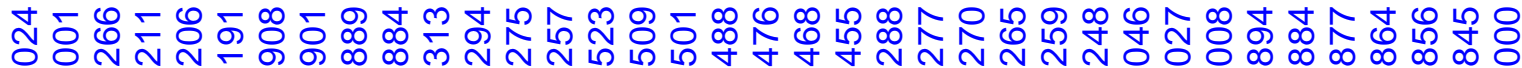
0000

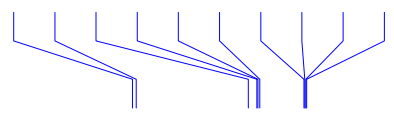

$\underset{\mathcal{N}}{\mathcal{N}}$

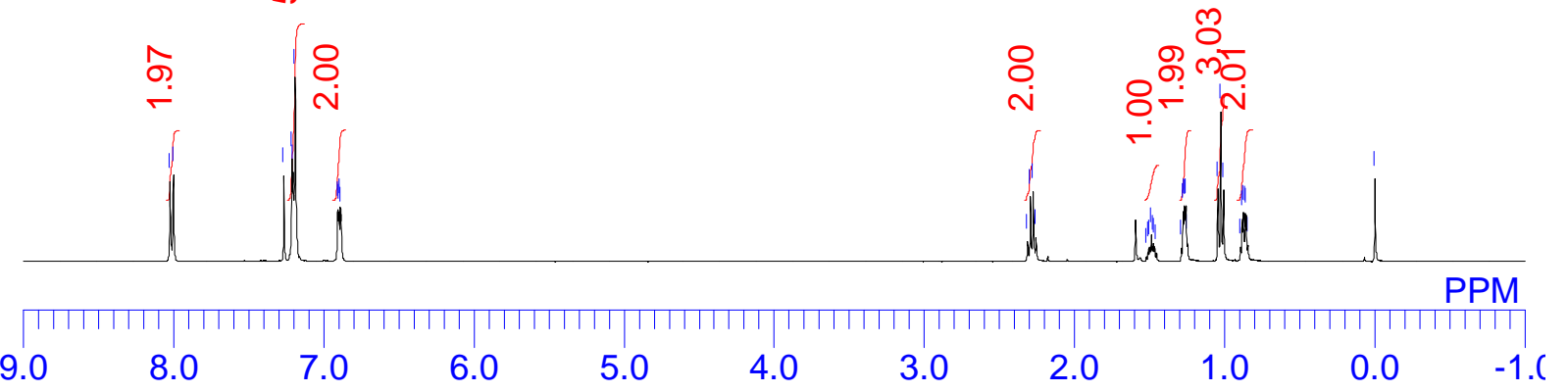

${ }^{13} \mathrm{C}\left\{{ }^{1} \mathrm{H}\right\} \mathrm{NMR}\left(100 \mathrm{MHz}, \mathrm{CDCl}_{3}\right)$

守

ชิ่

तิ

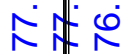

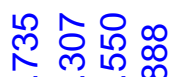

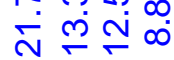
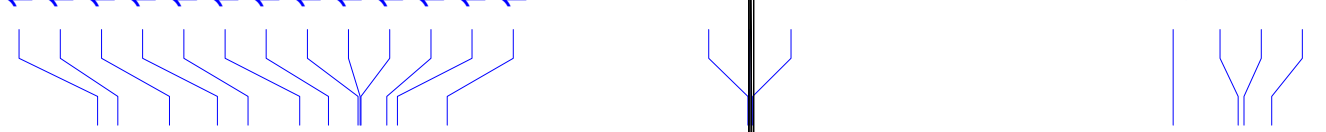

PPM

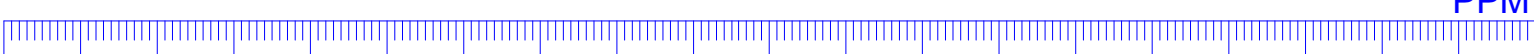

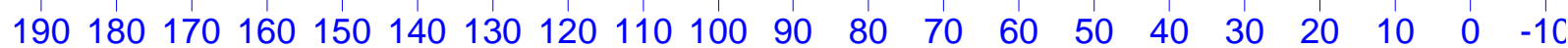


5-(4-methylbenzoyl)-6-phenyl-2H-pyran-2-one (7a)<smiles>Cc1ccc(C(=O)c2ccc(=O)oc2-c2ccccc2)cc1</smiles>

${ }^{1} \mathrm{H} \mathrm{NMR}\left(400 \mathrm{MHz}, \mathrm{CDCl}_{3}\right)$

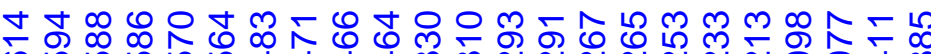

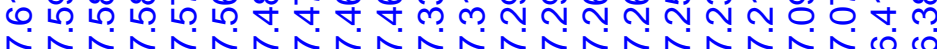

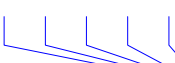

11

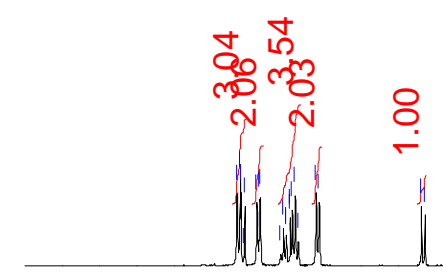

ले

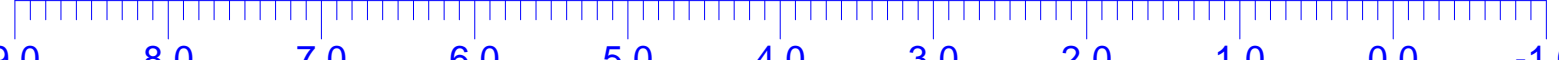

9.0

$8.0 \quad 7.0$

5.0

4.0

3.0

2.0

1.0

0.0

${ }^{13} \mathrm{C}\left\{{ }^{1} \mathrm{H}\right\} \mathrm{NMR}\left(100 \mathrm{MHz}, \mathrm{CDCl}_{3}\right)$

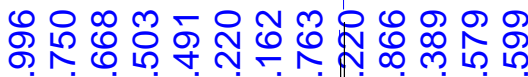

ن

공ㅇำ

กิ゚

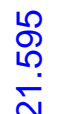

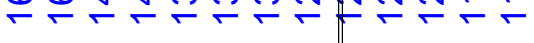

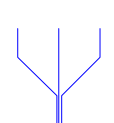

PPM

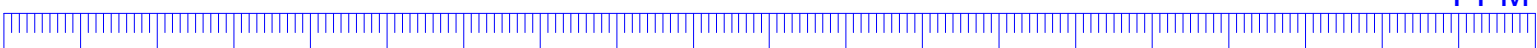

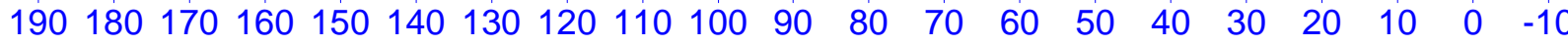


5-(4-methylbenzoyl)-4,6-diphenyl-2H-pyran-2-one (7b)<smiles>Cc1ccc(C(=O)c2c(-c3ccccc3)cc(=O)oc2-c2ccccc2)cc1</smiles>

${ }^{1} \mathrm{H}$ NMR (400 MHz, $\left.\mathrm{CDCl}_{3}\right)$

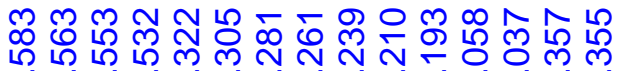

$\stackrel{\infty}{\stackrel{\infty}{N}}$

৪

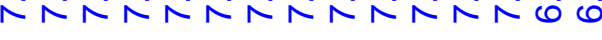

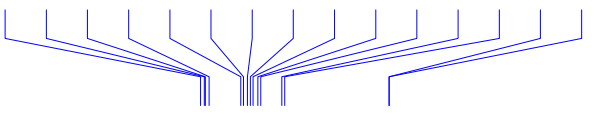

N
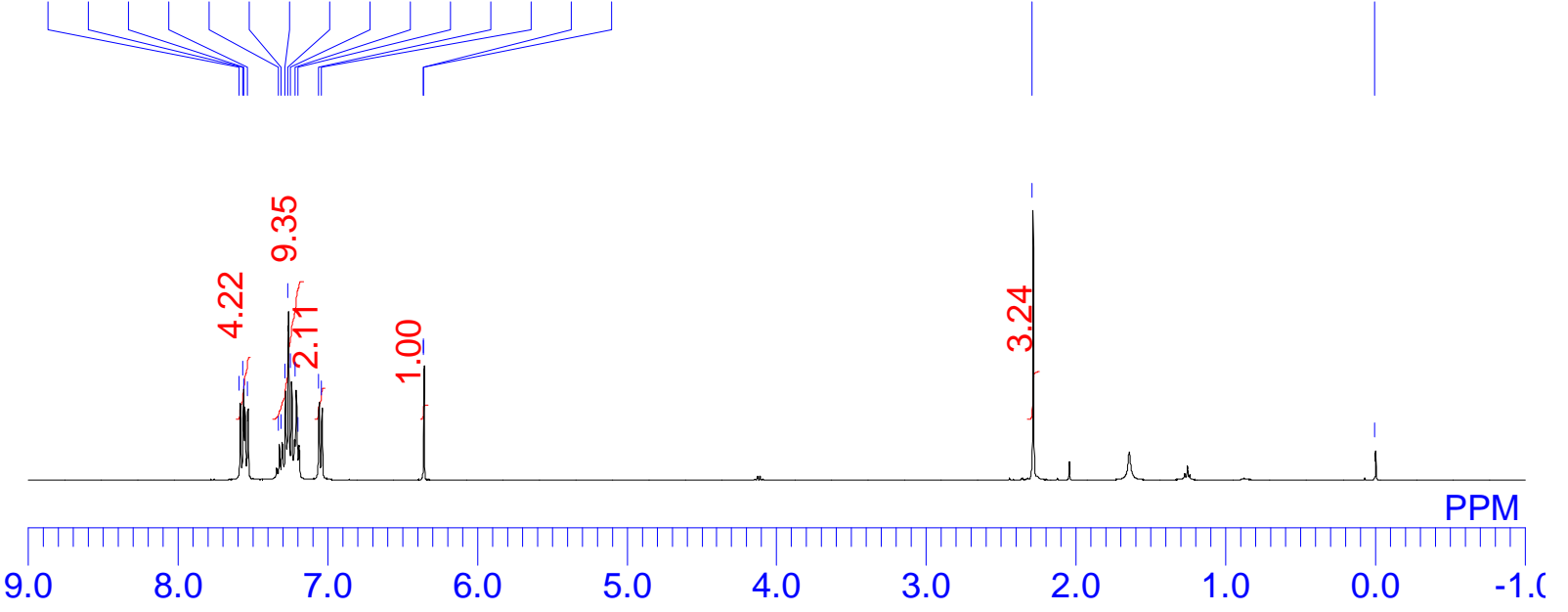

${ }^{13} \mathrm{C}\left\{{ }^{1} \mathrm{H}\right\}$ NMR $\left(100 \mathrm{MHz}, \mathrm{CDCl}_{3}\right)$

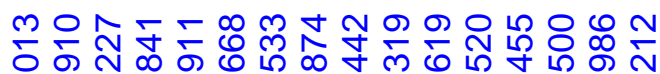
ซ่

LLLLLLLLU1JJUJJ

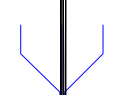

PPM

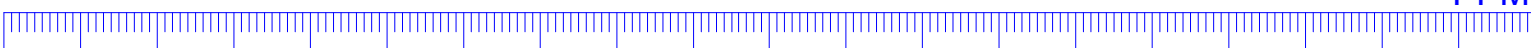

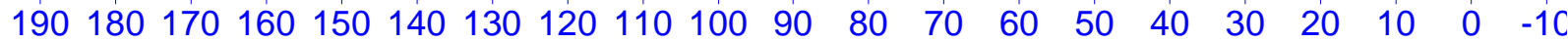


3-ethyl-5-(4-methylbenzoyl)-4,6-diphenyl-2H-pyran-2-one (7c)<smiles>CCc1c(-c2ccccc2)c(C(=O)c2ccc(C)cc2)c(-c2ccccc2)oc1=O</smiles>

${ }^{1} \mathrm{H}$ NMR (400 MHz, $\left.\mathrm{CDCl}_{3}\right)$
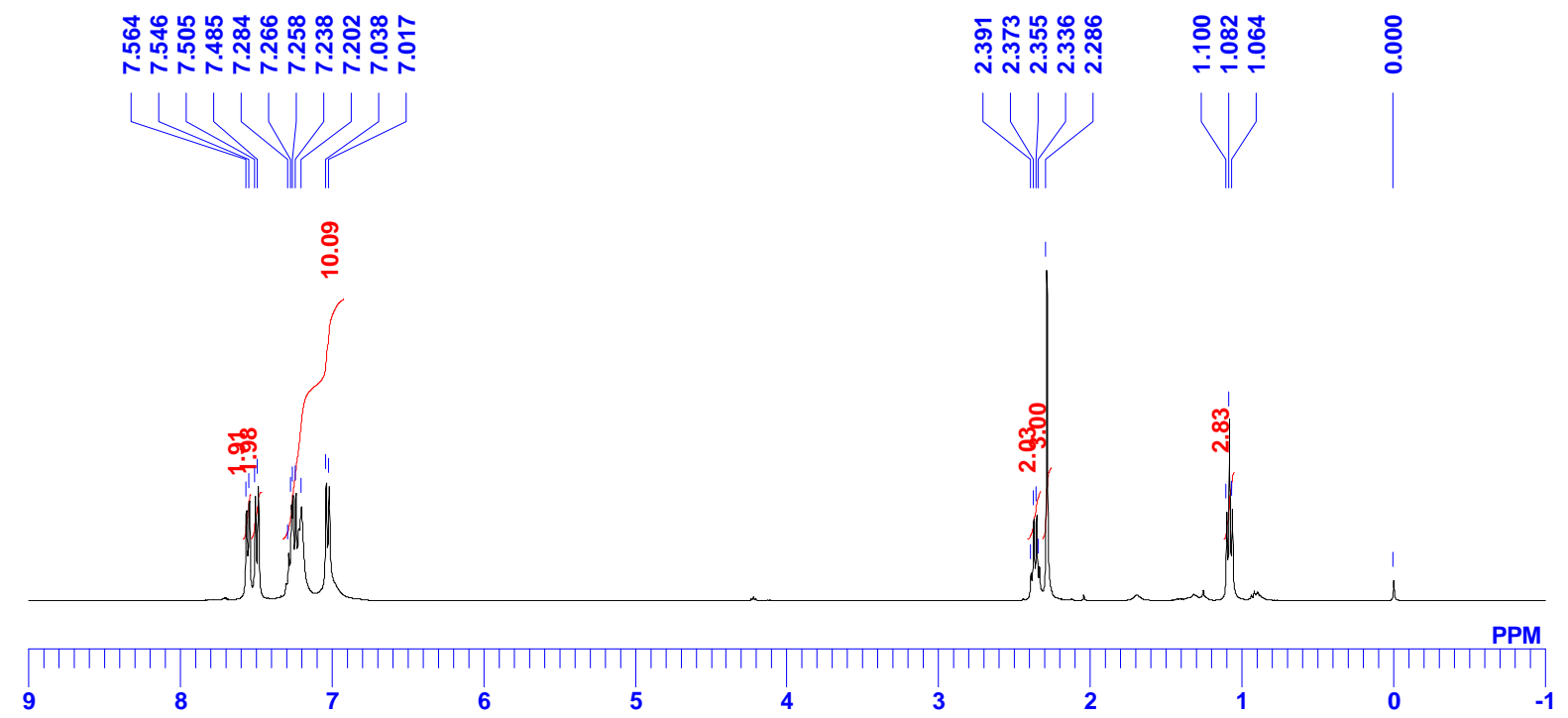

${ }^{13} \mathrm{C}\left\{{ }^{1} \mathrm{H}\right\}$ NMR $\left(100 \mathrm{MHz}, \mathrm{CDCl}_{3}\right)$

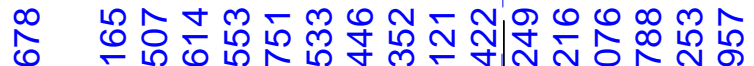

ฏ

긍요 웅

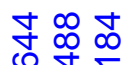

余食

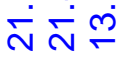
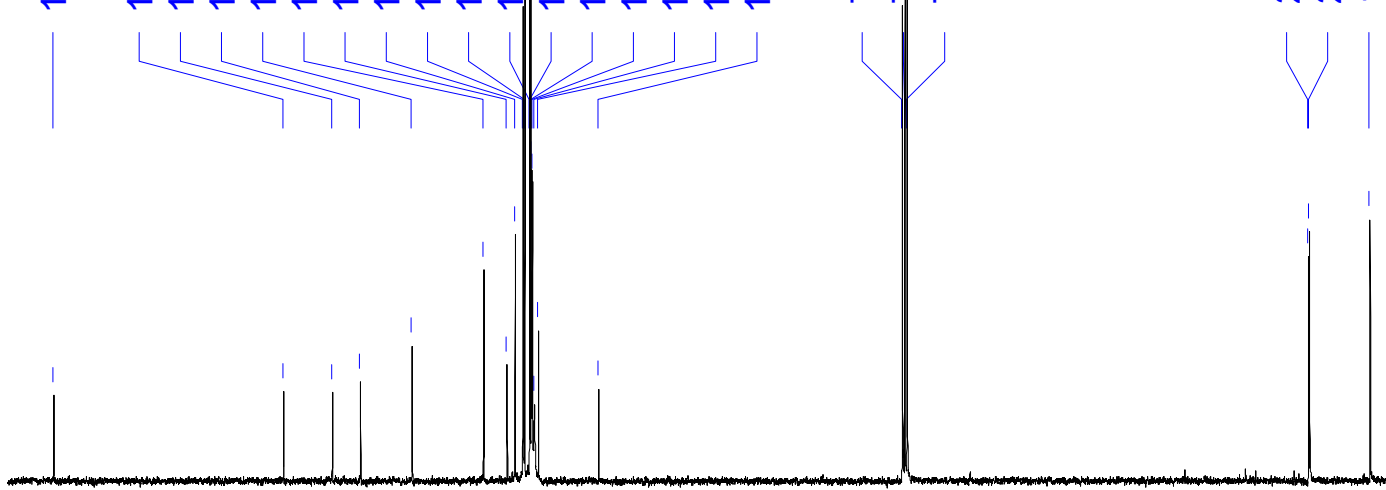

PPM

|

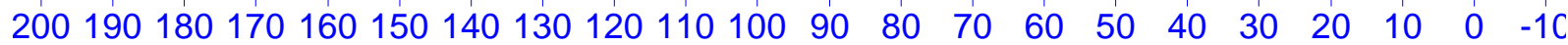


5-(4-methylbenzoyl)-3,4,6-triphenyl-2H-pyran-2-one (7m)<smiles>Cc1ccc(C(=O)c2c(-c3ccccc3)oc(=O)c(-c3ccccc3)c2-c2ccccc2)cc1</smiles>

${ }^{1} \mathrm{H} \mathrm{NMR}\left(400 \mathrm{MHz}, \mathrm{CDCl}_{3}\right)$
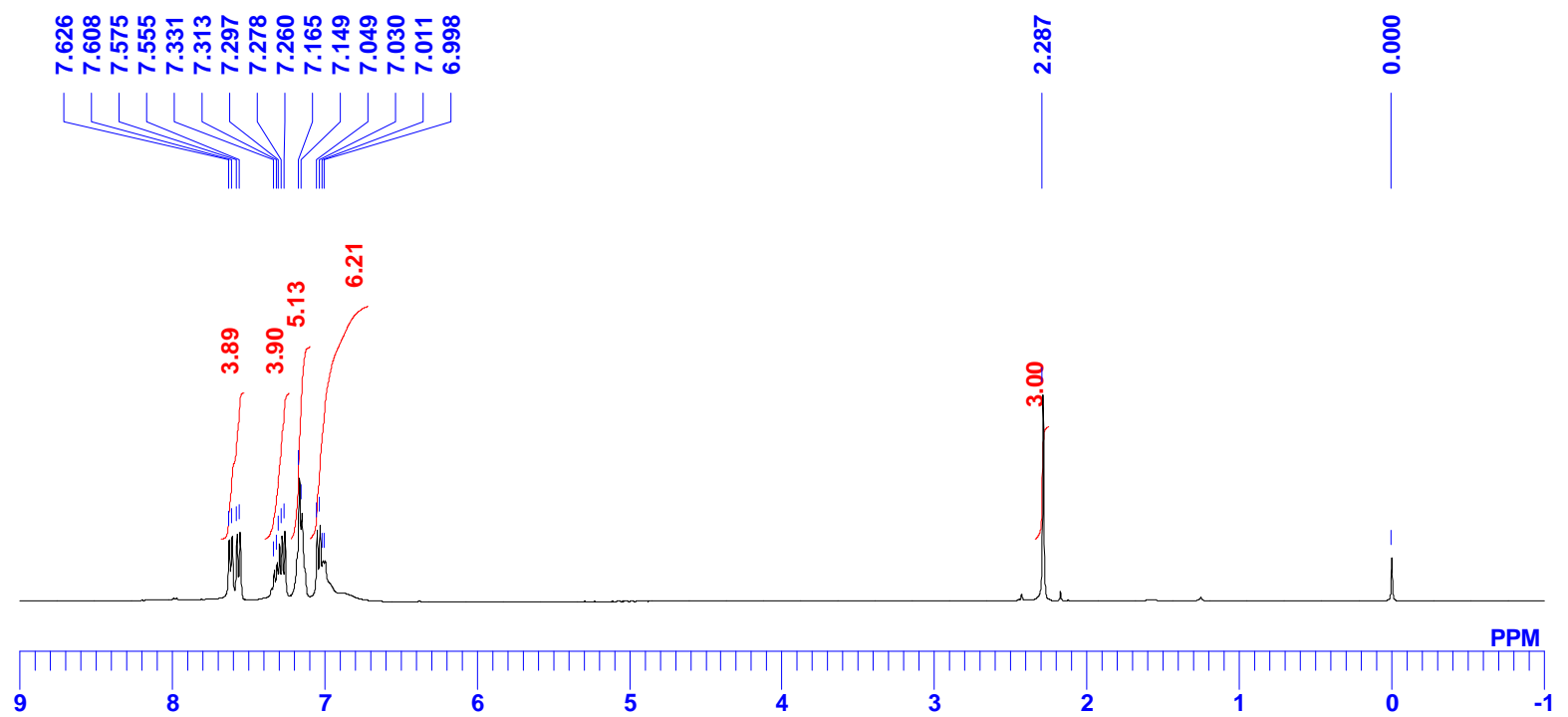

${ }^{13} \mathrm{C}\left\{{ }^{1} \mathrm{H}\right\}$ NMR $\left(100 \mathrm{MHz}, \mathrm{CDCl}_{3}\right)$

ஐ

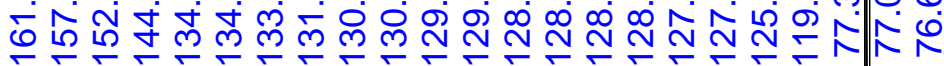

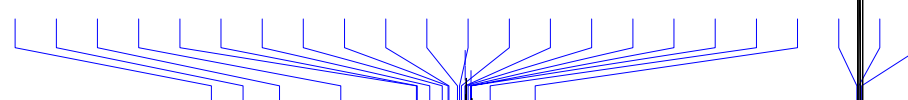


6-cyclopropyl-3-ethyl-5-(4-methylbenzoyl)-4-phenyl-2H-pyran-2-one (7l)<smiles>CCc1c(-c2ccccc2)c(C(=O)c2ccc(C)cc2)c(C2CC2)oc1=O</smiles>

${ }^{1} \mathrm{H}$ NMR $\left(400 \mathrm{MHz}, \mathrm{CDCl}_{3}\right)$
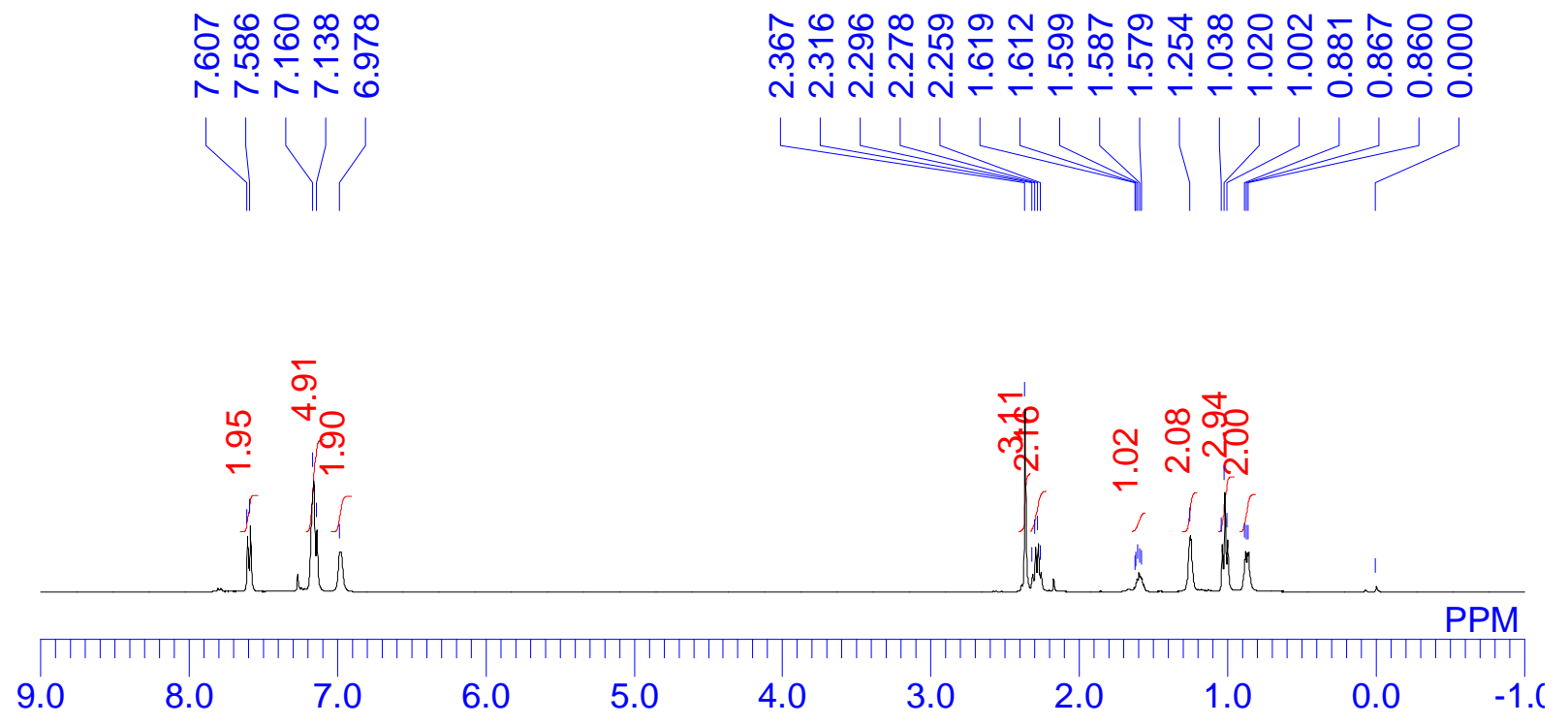

${ }^{13} \mathrm{C}\left\{{ }^{1} \mathrm{H}\right\}$ NMR $\left(100 \mathrm{MHz}, \mathrm{CDCl}_{3}\right)$

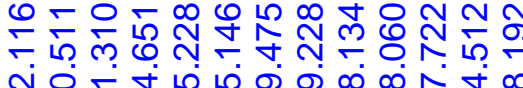

엄

तี

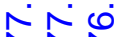

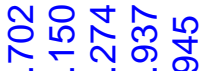
ลี่
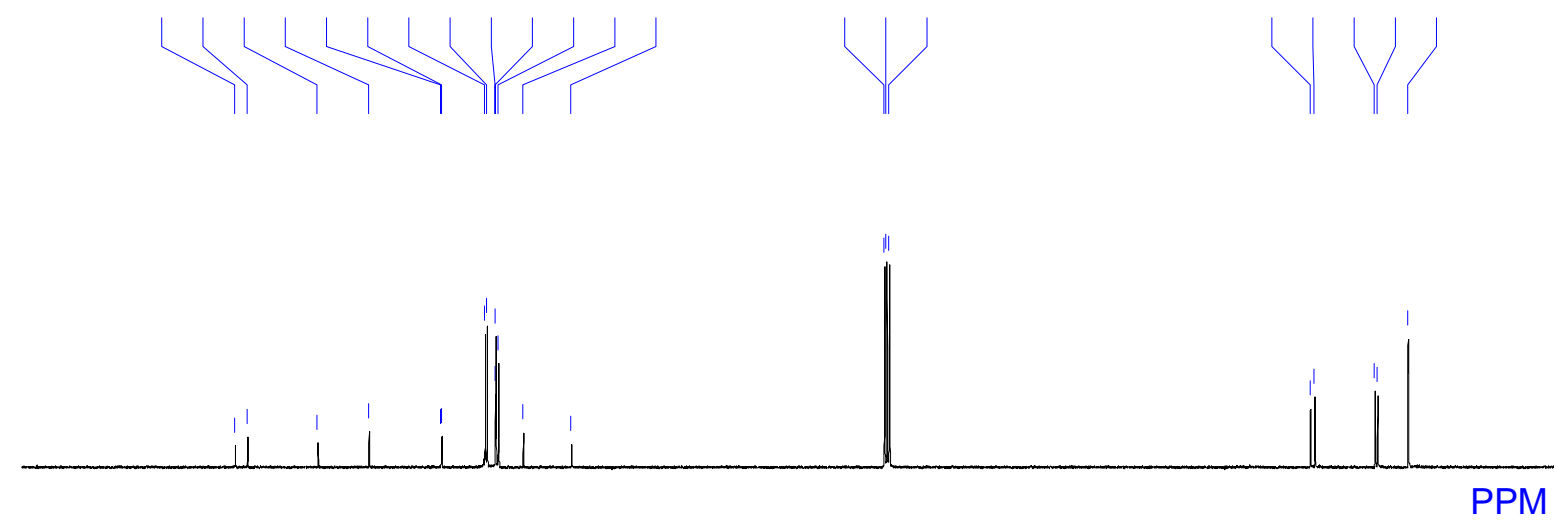

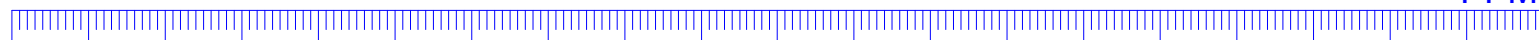

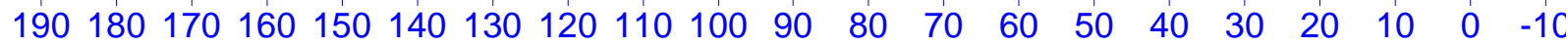


3,4,6-triphenyl-5-(phenylethynyl)-2H-pyran-2-one (8)<smiles>O=c1oc(-c2ccccc2)c(C#Cc2ccccc2)c(-c2ccccc2)c1-c1ccccc1</smiles>

${ }^{1} \mathrm{H} \mathrm{NMR}\left(400 \mathrm{MHz}, \mathrm{CDCl}_{3}\right)$

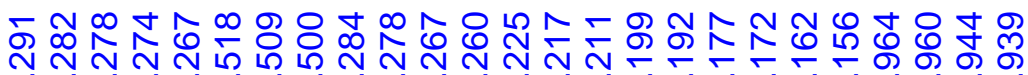
舟
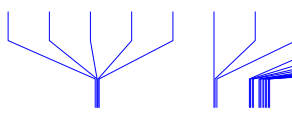

$\stackrel{\text { ñ }}{\text { m }}$

$\underset{\sim}{\stackrel{N}{\rightarrow}}$
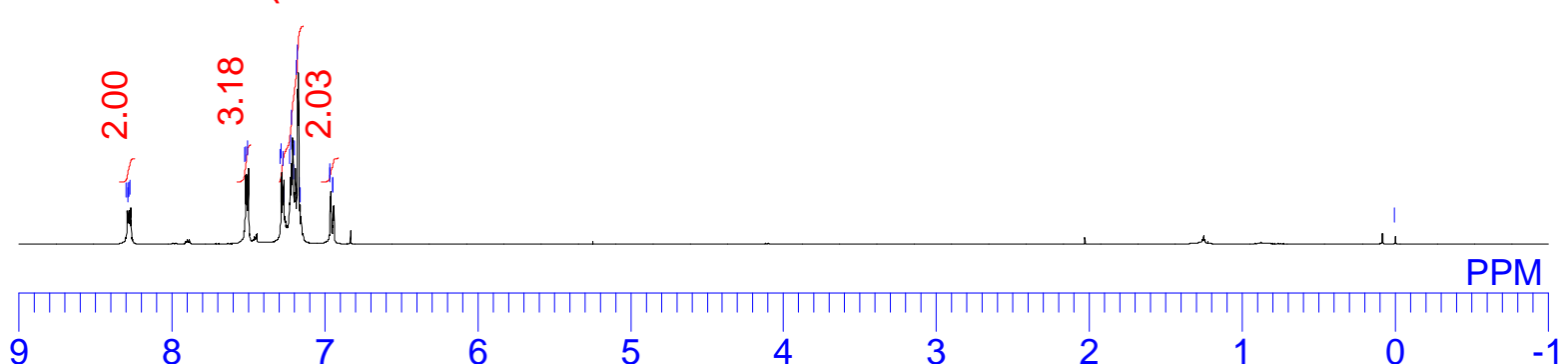

${ }^{13} \mathrm{C}\left\{{ }^{1} \mathrm{H}\right\} \mathrm{NMR}\left(100 \mathrm{MHz}, \mathrm{CDCl}_{3}\right)$

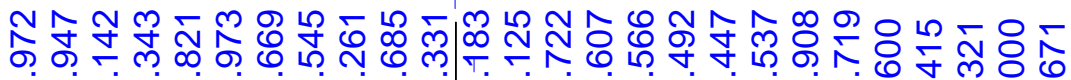
ه

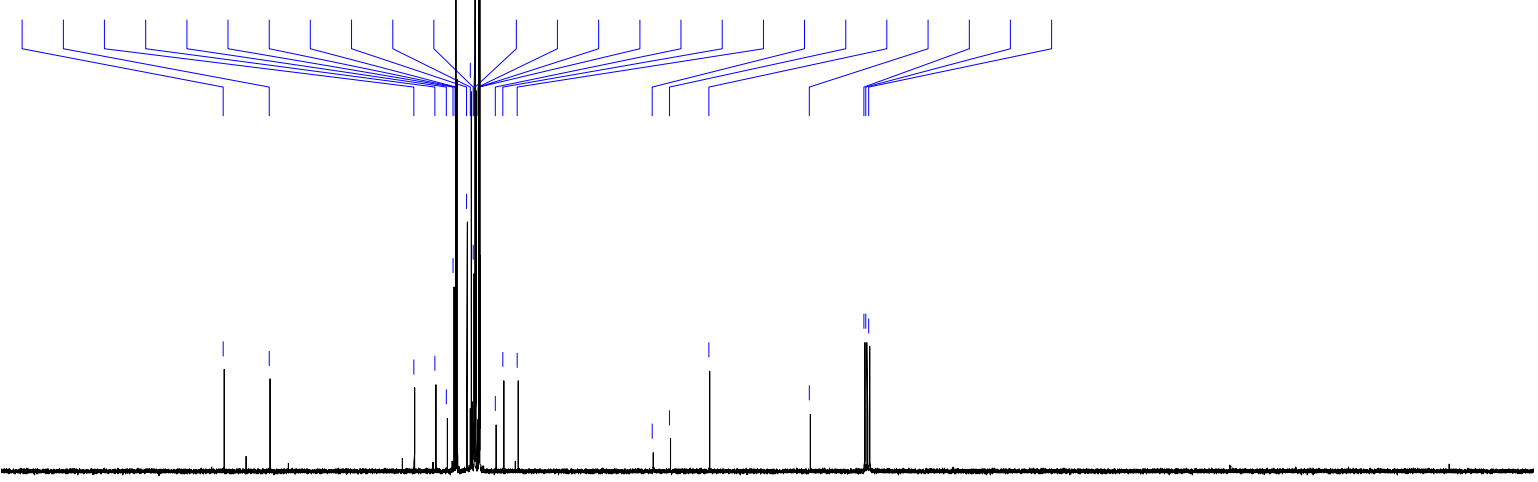

PPM

|

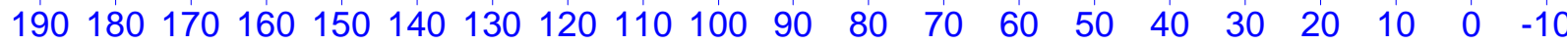


methyl (Z)-2-\{iodo(phenyl)methylene $\}$ butanoate (9)<smiles>CCC(C(=O)OC)=C(I)c1ccccc1</smiles>

${ }^{1} \mathrm{H}$ NMR (400 MHz, $\left.\mathrm{CDCl}_{3}\right)$
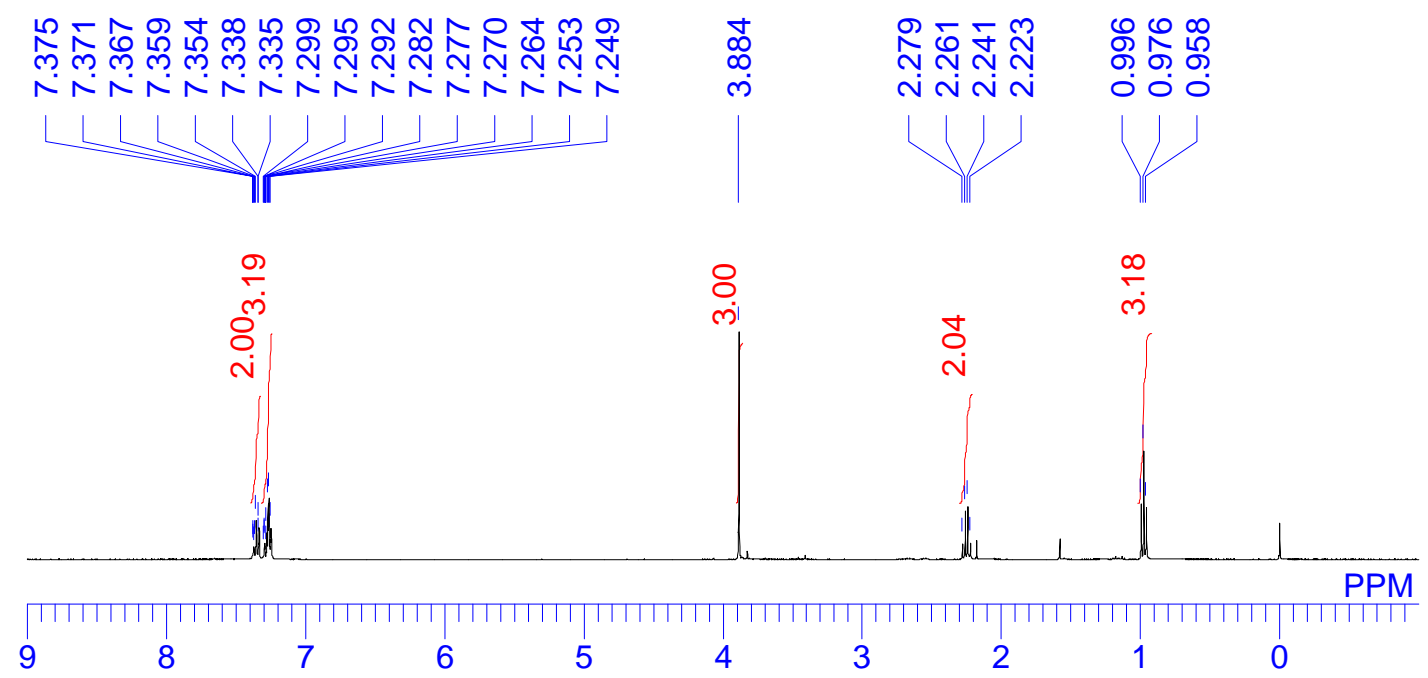

${ }^{13} \mathrm{C}\left\{{ }^{1} \mathrm{H}\right\}$ NMR $\left(100 \mathrm{MHz}, \mathrm{CDCl}_{3}\right)$

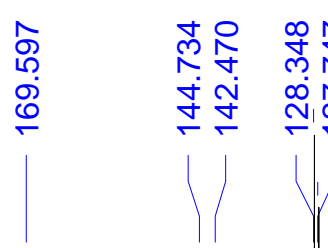

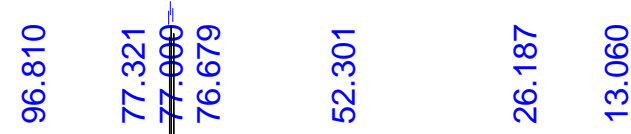

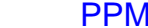

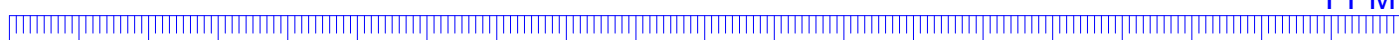

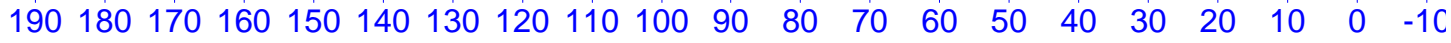

